\title{
Biomechanically Inspired Assistive Technology to Restore Movement of the Upper Limbs After Stroke
}

\author{
Ariel Thomas \\ West Virginia University, abthomas@mix.wvu.edu
}

Follow this and additional works at: https://researchrepository.wvu.edu/etd

Part of the Biomedical Engineering and Bioengineering Commons, Neuroscience and Neurobiology Commons, and the Physical Therapy Commons

\section{Recommended Citation}

Thomas, Ariel, "Biomechanically Inspired Assistive Technology to Restore Movement of the Upper Limbs After Stroke" (2021). Graduate Theses, Dissertations, and Problem Reports. 8217.

https://researchrepository.wvu.edu/etd/8217

This Dissertation is protected by copyright and/or related rights. It has been brought to you by the The Research Repository @ WVU with permission from the rights-holder(s). You are free to use this Dissertation in any way that is permitted by the copyright and related rights legislation that applies to your use. For other uses you must obtain permission from the rights-holder(s) directly, unless additional rights are indicated by a Creative Commons license in the record and/ or on the work itself. This Dissertation has been accepted for inclusion in WVU Graduate Theses, Dissertations, and Problem Reports collection by an authorized administrator of The Research Repository @ WVU.

For more information, please contact researchrepository@mail.wvu.edu. 


\title{
Biomechanically Inspired Assistive Technology to Restore Movement of the Upper Limbs After Stroke
}

\author{
Dissertation submitted to the West Virginia University Health Science Center Neuroscience
}

Program in partial fulfillment of the requirements for the degree of

Doctor of Philosophy in Neuroscience

Valeriya Gritsenko, Ph.D., Chair

Shou Wang, Ph.D.

Victor Finomore, Ph.D.

Sergiy Yakovenko, Ph.D.

Jessica Allen, Ph.D.

Department of Neuroscience

Morgantown, West Virginia

2021

Keywords: Stroke, Biomechanics, Reaching

Copyright 2021 Ariel B. Thomas 


\begin{abstract}
Biomechanically Inspired Assistive Technology to Restore Movement of the Upper Limbs After Stroke
\end{abstract}

Ariel Thomas

A stroke often damages the neural structures responsible for movement. Stroke is a heterogeneous disease, affecting each survivor differently. There are common motor features of a stroke, but even these features vary across time as an individual proceeds through different stages of recovery. The different ways in which stroke motor impairment can present itself are often overlooked, but these differences are fundamental to the understanding of the disease and its recovery. When motor assessments are capable of acquiring information necessary to parse out a detailed profile of each stroke case, this will lead to an improved neuromechanical understanding of the disease and an improved application of personalized rehabilitative techniques and assistive devices.

In my research, the key approach was to understand the stroke-related neuromechanical disruption at the level of joint torques and then selectively restore function using a wearable assistive device. In my first aim, I investigated post-stroke upper-limb motor deficits in terms of muscle torques related to movement and gravity compensation. Using biomechanical simulations of detailed kinematic data from the upper limbs, I was able to separate active muscle torques into profiles of activity that stabilize the limb against gravity from those that are used to control multi-joint movement. The results of this work found that elements of active muscle torque provide a more sensitive measure of impairment than angular kinematic measures. In my second aim, I designed a Functional Electrical Stimulation (FES) protocol using concepts of neuromechanics to improve coordination and promote neuroplasticity. I hypothesized that FES used to support the weight of the arm against gravity in upper-limb motor impaired stroke subjects will not interfere with the execution of reaching movements and will improve grasping performance. The goal of this aim was to design and test a biomechanical model-driven FES intervention to reduce shoulder abduction and flexion loads due to gravity and assist with a reaching movement. Rather than use an exoskeleton to offer support, we used profiles of gravity-compensation torque, identified in Aim 1, as the design for the FES stimulation to support the weight of the arm during unrestrained center-out and return movements. This improved movement speed, grasping performance, and distal muscle coordination while not interfering with reaching trajectory in our sample of 4 stroke survivors. 
Following this application, we investigated the effect of extended use of assistive FES on the induced movement and on the glenohumeral joint of the shoulder. Movement induced by FES decreased did not decrease over time and the stimulation-induced effect on the glenohumeral joint was still present after a 1-1.5 hour session in controls, or in a 30 minute session in stroke participants. This supports the effectiveness of this technique used for movement assistance. Although this data is preliminary, the results in our last aim also suggest that this application of FES may strengthen muscles associated with the glenohumeral joint, which in turn prevents subluxation injury. We believe this application has potential for the improvement of assistive technology after stroke. 


\section{Acknowledgements}

I would like to thank the study participants for generously giving their time; I am exceedingly grateful for the technical support expertly provided by R. Hardesty, and M. Boots; and I would like to thank A. Korol, M. Yough, S. Bahdasariants, A. Smith and M. Barr for their help with recruitment, data collection, literature evaluation, and editing. Finally, I would like to thank S. Yakovenko for his insight on these projects and for creating a multi-laboratory discussion platform and writing group where we could exchange our ideas and help one another. I would also like to thank the members of my committee, J. Allen, S. Wang, and V. Finomore for their guidance and revisions. Finally, I would like to thank my advisor and committee chair, V. Gritsenko, for her guidance and support throughout my research.

\section{Funding}

VG was supported by grants P20GM109098 and P30GM103503 from the National Institute of General Medical Sciences (https://www.nigms.nih.gov). ABT was supported by the training grant T32AG052375 from the National Institute of General Medical Sciences and National Science Foundation grant \#2014645. The funders had no role in study design, data collection and analysis, decision to publish, or preparation of the manuscript. 


\section{TABLE OF CONTENTS}

\section{CHAPTER 1. BACKGROUND}

INTRODUCTION TO THE CURRENT STATE OF STROKE REHABILITATION ........... 1

Part I: Anatomy and Neuromechanics of Stroke ................................................... 2

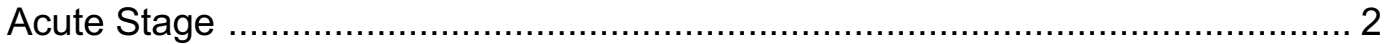

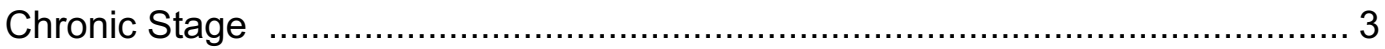

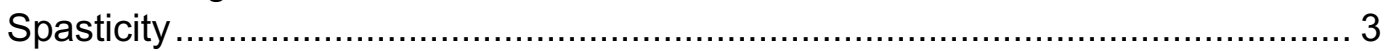

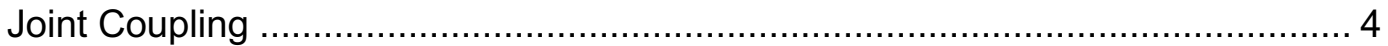

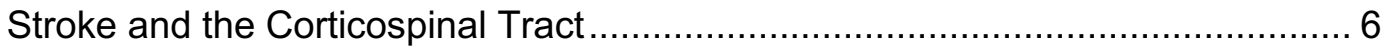

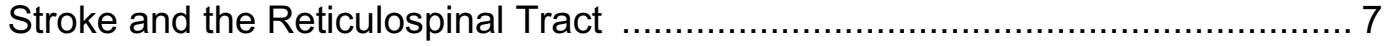

Stroke and the Vestibulospinal Tract ......................................................... 9

Stroke and the Supplementary Motor Area and Premotor Cortex ..................... 10

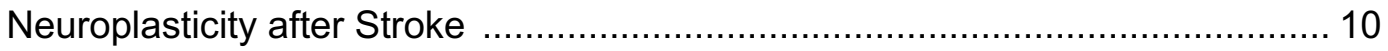

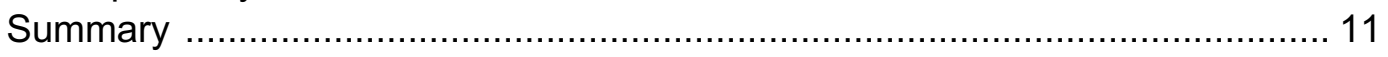

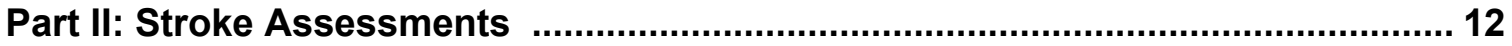

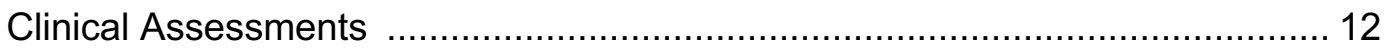

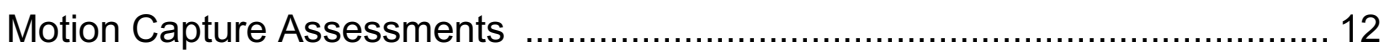

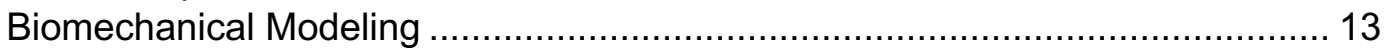

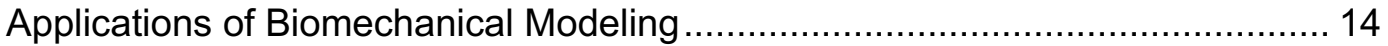

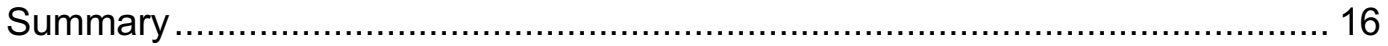

Part III: Personalized rehabilitative techniques and assistive devices ...................17

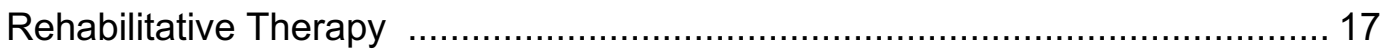

Clinical Use of Functional Electrical Stimulation ....................................... 18

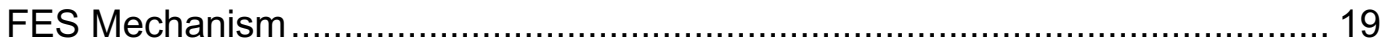

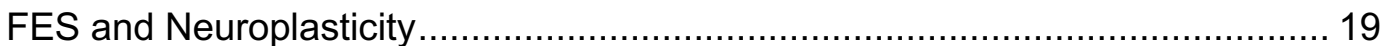

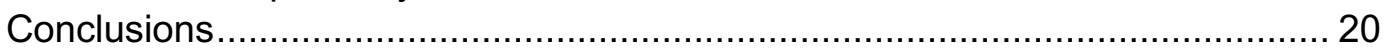

\section{CHAPTER 2. STROKE MOVEMENT ANALYSIS}

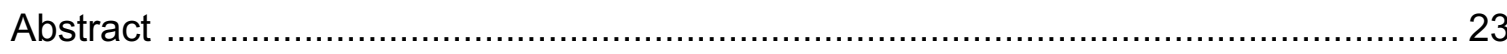

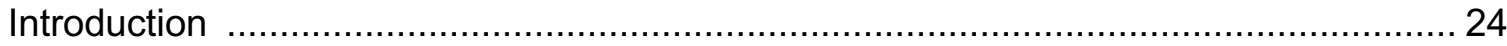

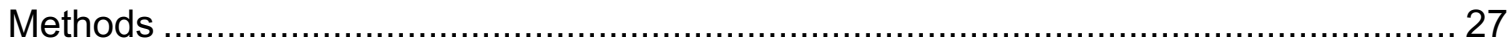

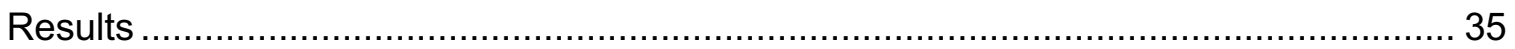

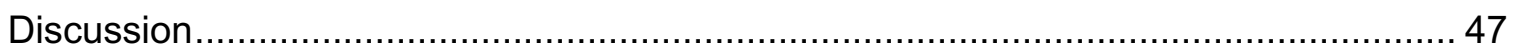

\section{CHAPTER 3. DESIGNING AN ASSISTIVE DEVICE FOR STROKE REHABILITATION}

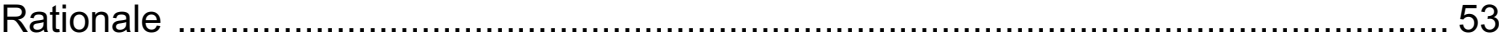

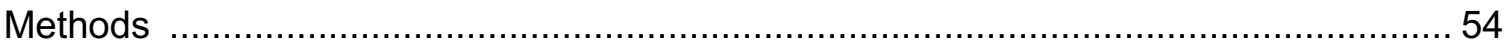

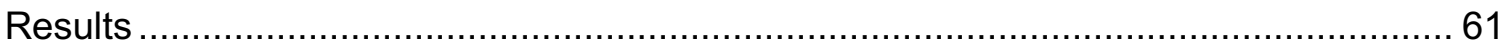

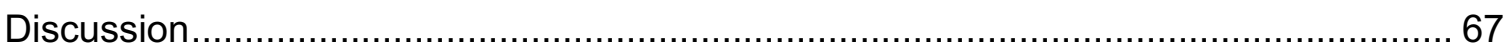


CHAPTER 4. EXAMINING FATIGUE AND THE IMPACT ON THE GLENOHUMERAL JOINT FOR SHOULDER FES

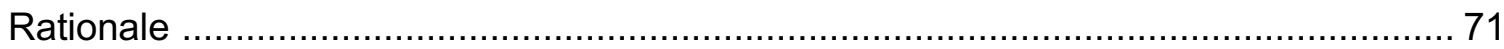

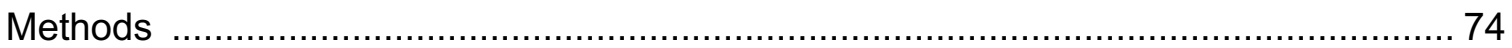

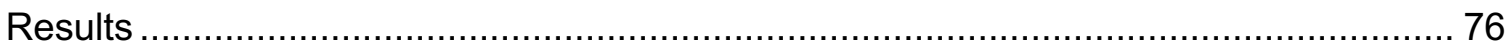

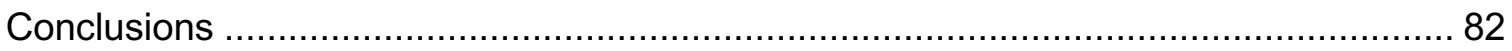

CHAPTER 5. CONCLUSIONS AND FUTURE DIRECTIONS

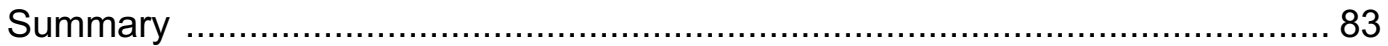

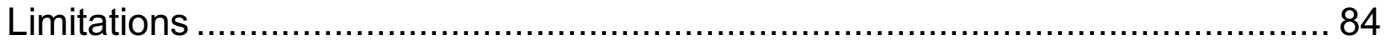

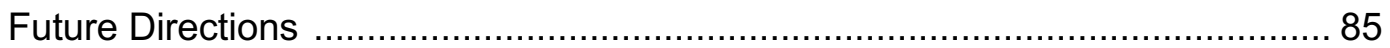

Advancements in Control Mechanisms for FES ................................ 85

Cost-Effective sensors for Wearable Technologies .............................. 86

Model-based Feedback Control ..................................................... 88

Use of co-activation in antagonistic muscles to stabilize control of upper limbs .................................................... 88

Combination of FES with Rehabilitation Robotics ............................ 89

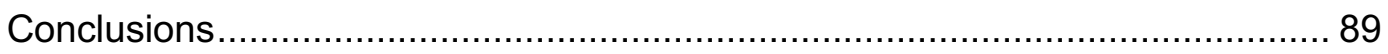

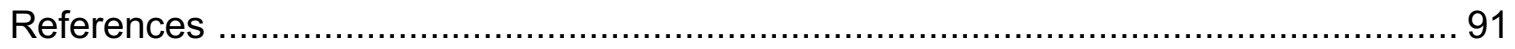




\section{List of Tables and Figures}

\section{Chapter 2}

Table 1. Characteristics of individuals with stroke.

Figure 1. Standardized reaching in virtual reality.

Figure 2. Kinematic and torque profiles of four participants.

Supplemental Figure S1. Comparison of angular acceleration and MTd profiles.

Supplemental Figure S2. Center-out reaching by participants with stroke.

Figure 3. Performance index for each movement direction per participant with stroke

Supplemental Figure S3. Performance index for each movement direction per participant with stroke including angular acceleration and MTd

Figure 4. Coefficient of determination $\left(R^{2}\right)$.

Supplemental Figure S4. Coefficient of determination (R2) including angular acceleration and MTd.

Figure 5. Cluster analysis of the coefficient of determination.

Supplemental Figure S5. Cluster analysis of the coefficient of determination based on angular acceleration and MTd.

\section{Chapter 3}

Table 1: FES Reaching Task Participant Information

Figure 1. Gravitational Muscle Torque Profiles used to design Gravity Support FES Parameters

Figure 2. Functional electrical stimulation of the shoulder during reaching

Table 2. Muscle Descriptions

Figure 3. Reach Trajectories

Figure 4. Movement Duration

Figure 5. Grasping Performance

Figure 6. Control Muscle Activity (EMG)

Figure 7. Stroke Muscle Activity (EMG)

Figure 9. Stimulation Fatigue

\section{Chapter 4}

Table 1. Participants

Figure 1. Shoulder ultrasound measurements.

Figure 2. Changes in shoulder angle for healthy controls and stroke subjects

Figure 3. FES induced change in shoulder angle per $\mathrm{mA}$ of stimulation for two stroke participants

Figure 4. Changes in glenohumeral distance and height with FES in healthy controls.

Table 2. Force measurements for stroke participants 


\section{List of Abbreviations}

Randomized Control Trials (RCTs)

Corticospinal Tract (CST)

Principle Component Analysis (PCA)

Activities of Daily Living (ADLs)

Fugyl-meyer Assessment (FMA)

$\mathrm{NIH}$ stroke scale (NIH SS)

Primary Motor Cortex (M1)

Reticulospinal Tract (RST)

Pontomedullary Reticular Formation (PMRF)

Vestibulospinal Tract (VST)

Middle Cerebral Artery (MCA)

Electromyography (EMG)

Magnetic Resonance Imaging (MRI)

Electroencephalography (EEG)

Supplementary Motor Area (SMA)

Premotor Cortex (PMC)

Spike Timing-Dependent Plasticity (STDP)

Long Term Potentiation (LTP)

Long Term Depression (LTD)

Wolf Motor Function Test (WMFT)

Action Research Arm Test (ARAT)

Constraint-induced Movement Therapy (CIMT)

Functional Electrical Stimulation (FES)

Neuromusclular Electrical Stimulation (NMES)

Cyclic Neuromuscular Electrical Stimulation (cNMES)

Degree of Freedom (DOF)

Muscle Torque (MT)

Gravitational component of Muscle Torque (MTg)

Dynamic component of Muscle Torque (MTd) 


\section{Chapter 1 - Background}

The most widely recognized long-term consequence of stroke is motor impairment. This typically affects the unilateral control of movement on the side opposite to the neuronal insult and affects about $80 \%$ of patients (Langhorne et al. 2011). Following acute stroke, or the weeks immediately following a stroke, approximately $77 \%$ and $72 \%$ of patients demonstrate motor deficits in their upper and lower limbs, respectively (Lawrence et al. 2001). Many stroke patients do not recover their full mobility even after years of physical therapy. Stroke is the leading cause of long term disability in the US, 50\% have lasting motor dysfunction (Benjamin et al. 2019). The initial hemiparesis which follows stroke will recover in many cases, whether by spontaneous or rehabilitation-induced mechanisms. However, long term motor deficits due to hemiparesis persist in many cases and have few effective strategies for treatment. A 2014 Cochrane review of Randomized Controlled Trials (RCTs) showed that there was not enough evidence to show that traditional physical therapy was having any effect on improving upper limb function after stroke (Pollock et al. 2014a). It is unclear whether this ineffective outcome was due to major problems in stroke rehabilitation, or a problem with the assessment of stroke disability. Some researchers attribute the root of this problem to the large divide between neuroscience research and clinical translation, summarized well in this statement by neurologist John Krakauer (Krakauer and Carmichael 2017a).

"The current crisis in neurorehabilitation is not because existing therapies have not been properly tested in RCTs but because existing and new experimental therapies themselves are often ill conceived from a biological and mechanistic standpoint. RCTs should not be conducted until more mechanistically informed therapies based on scientific principles have been devised."

This idea of neuromechanically informed clinical applications is not new, yet it often has difficulty being accepted and used widely by the medical community. RCT design is based on current medical approaches, which are likely based on medical traditions rather than current scientific evidence. It may be that current RCTs are designed on outdated principles, are given at the wrong time or in too low frequency of application, or are quantified using measures of improvement that are inadequate for evaluating the treatment. The purpose of my research, therefore, is to apply the most current neuromechanical understanding of stroke for both the evaluation and treatment of stroke movement disorder. 


\section{Part I: Anatomy and Neuromechanics of Stroke}

Stroke is a heterogeneous disease, affecting each survivor differently. There is a complexity to the production of movement that we currently do not fully understand. Damage to the neural structures responsible for movement production has been documented for centuries, however primarily subjectively. This investigation is improving dramatically with recent advancements in tools for objective assessment, such as motion capture, neural recordings, and imaging. What we are learning through the quantitative evaluation of stroke and stroke recovery should be used to improve rehabilitation strategies.

The restoration of motor function after stroke is thought to involve compensation through the upregulation of other descending pathways, a form of neuronal reorganization and neuroplasticity (Dimyan and Cohen 2011). Importantly, depending on the motor behaviors that are recruited during the recovery period this neural reorganization could be either beneficial or maladaptive impairing further rehabilitative progress (Kerr et al. 2011). During the acute phase, motor disorder presents in the upper limbs as a flaccid paralysis with diminished muscle activity (Lawrence et al. 2001). During recovery, the muscle activity that returns can be poorly controlled and present with painful spasticity. This section will summarize current theories on the neural mechanisms behind the transition from flaccid paralysis to spasticity and joint coupling during post-stroke recovery.

\section{Acute Stage}

The primary motor pathways responsible for dexterous control, the corticospinal tract (CST) is often the most directly impacted motor pathway following a stroke. The CST is responsible for precise, independent control of single joints, and damage severely impairs the fine motor skills that are essential for interacting with complex environments (Yoo et al. 2014). Muscle weakness and loss of dexterity are two of the primary clinical signs in stroke-related hemiparesis. Reach and grasp, two of the most significant tasks of the upper extremities, are often the most affected upper limb ability. The deficits of reach and grasp after stroke consist of premature hand closure, inadequate aperture, dysmetria, segmented movements, and slowness of movement (Pelton et al. 2012). Cognitive impairment such as problems with executing planned tasks and problems with procedural memory can also greatly affect movement. These are most prevalent in the acute stage but can persist in some people. After a stroke, it can be difficult to remember the steps of a skilled task (Brodal 1973). It is important to consider cognitive impairment when assessing motor disability, as it may influence perceived motor deficits in skilled tasks. 
Chronic Stage

After initial CST injury, there are secondary adaptive changes in the brain and spinal cord (Puig et al. 2013; Schaechter et al. 2009). While muscle weakness is the dominant motor symptom in the first month after the stroke, spasticity and joint coupling become more prominent in the chronic stage. For instance, flexor muscle group activity tends to become stronger than extensor activity in this stage (Kamper et al. 2006; Kline et al. 2007), to the point of producing flexion instead of extension when attempting extension tasks (Lan et al. 2017; Miller and Dewald 2012). While grasping is still significantly affected, the weakness may be replaced in some individuals by strength and an increasingly rigid and flexed resting posture. While grip strength is improved in these patients, the fine movement control and dexterity may not return. Individuals with severe motor impairment in the chronic stage often demonstrate involuntary increases in their grasping strength when attempting to open their hand. Importantly, this is not reflective of the recovery course of all patients, but it is consistent enough in stroke survivors to affect millions of people worldwide. This reinforces the idea that care must be personalized, and that rehabilitative trials must have enough precision in their measurements and analyses to distinguish cases presenting with spasticity and examine which rehabilitation approaches are most effective in these cases.

\section{Spasticity}

Spasticity is a motor disorder characterized by a velocity-dependent increase in muscle tone with hyperactive reflexes (Burke et al. 2013). Spasticity is considered to be a common feature of stroke recovery, although it only develops in about a third of patients with stroke hemiparesis. The causal mechanisms for spasticity continue to be debated. The most widely accepted theory is that there are immediate and delayed changes in spinal cord activity due to the loss of descending input onto components of the stretch reflex arc. Several theories have been proposed, including the loss of presynaptic inhibition of la spindle afferents and increased group II spindle afferent excitation (Nardone and Schieppati 2005), and increased tonic input onto a range of proprioceptive neurons from descending extrapyramidal tracts, most notably the medial reticulospinal tract (Chen et al. 2019; Li et al. 2019; Sangari and Perez 2019).

In the past, poor coordination after stroke was attributed to spasticity. Therapies were designed to target spasticity to improve movement. However, results from clinical trials have shown that treating spasticity is useful for the alleviation of muscle pain but does not improve voluntary 
control in the upper limbs (Sheean 2002). Current research suggests that the increased gain of stretch reflexes is not the cause of spasticity, but instead the two symptoms are independent. Performance measures in fast multi-joint reaching movements in patients with hemiparesis are not related to the presence of spasticity (Beer et al. 2000; Ellis et al. 2017). While there is evidence to support the role of increased descending reticulospinal activity as the cause of increased muscle tone and hyperexcitable tendon jerks (the hallmarks of spasticity), these two features may not always occur together (Fellows et al. 1994a, 1994b).

Joint Coupling

Early post-stroke recovery also shows the emergence of joint coupling, which can persist through years after stroke (Bourbonnais et al. 1989; Ellis et al. 2011; Owen et al. 2017; Twitchell 1951). In post-stroke joint coupling, the ability to provide specific control of movement at each joint is replaced by highly stereotyped patterns of multi-joint movements caused by involuntary muscle activation (Brunnstrom 1966). One common joint coupling after stroke, the 'flexion synergy', occurs in the arm contralesional to stroke, where lifting the arm at the shoulder (i.e., shoulder abductor activation) drives simultaneous and involuntary activation of the elbow, wrist and finger flexors (Bowden et al. 2010; Dewald et al. 1995; Ellis et al. 2005, 2017; Levin 1996; Miller and Dewald 2012; Sukal et al. 2007; Twitchell 1951). This synergy reduces the ability to reach with the arm and impairs hand opening (Dewald et al. 2001; Roh et al. 2013).

A major hallmark of the flexion synergy is that it only occurs during self-initiated movement, and does not occur when the arm is passively raised by a therapist or assistive device (Ellis et al. 2017). Muscle activity involved in the flexion synergy is not due to the activity of the stretch reflex circuit, as previously thought (Bhadane et al. 2015). Elbow and more distal muscle activation for the hand and wrist occurs simultaneously with activation of the shoulder and before the onset of movement, which also discounts spasticity as the cause (Ellis et al. 2017; Sangani et al. 2007). The waveforms of the simultaneous shoulder and elbow activations are also similar, further providing evidence that this activity is driven by the same neural mechanisms, rather than a biomechanically driven reaction to shoulder movement (Sangani et al. 2007). Flexor synergies are task-dependent, in that they are increasingly expressed as voluntary shoulder muscle activity increases. This is often tested by increasing the load on the shoulder (Beer et al. 1999, 2004; Ellis et al. 2008, 2009; Sukal et al. 2007). The harder a subject must work to abduct the arm, the greater the activation of the other muscles coupled through the synergy. Many studies have shown that the flexion synergy severely limits movement. For 
example, it limits the elbow extension and hand opening necessary for object acquisition (Lan et al. 2017; Miller and Dewald 2012; Sukal et al. 2007). It becomes harder to break free from this synergy when the abduction load on the shoulder increases. Gravity support of the arm using robots has been shown to help a stroke subject regain individual joint control, and abduction load training has been tested as a rehabilitative strategy, with varying results (Ellis et al. 2009, 2018; Lan et al. 2017; McPherson et al. 2018; Miller and Dewald 2012).

An interesting question is whether the goal of rehabilitation should be restitution, i.e., returning towards the pre-stroke level of motor control and strength, or compensation, i.e., maximizing the use of residual motion to accomplish a task (Levin et al. 2009; Rothi and Horner 1983). After a stroke, restitution occurs as the ipsilesional motor cortex regains cortical control of motor function (Kim et al. 2006; Schaechter et al. 2009), while driving movement of the arm via contralesional pathways (originating from secondary motor areas or using reticulospinal or vestibulospinal activity) can be considered compensation (Jang et al. 2013; Levin et al. 2016). While a person may not be regaining what is considered 'normal' motor behavior, the question to ask is whether their compensatory behavior good enough for their activities of daily living (ADLs). As a compensatory mechanism, abnormal synergies associated with spasticity could be taken advantage of and trained in order to improve voluntary control, such as by increasing grip strength. On the other hand, is relying on the compensatory behavior impeding their ability to recover fully? Compensation may allow individuals to generate greater activity at the more proximal portions of the arm, although at the detriment of individual joint control and distal hand function, especially hand opening (Lan et al. 2017), as well as elbow extension and shoulder flexion (Sukal et al. 2007). The Fugl-Meyer Assessment (FMA) was developed specifically to be able to document the ability to independently control joints outside of the flexion and extension synergies. This is an observable, quantifiable phenomenon that has the potential to be directly treated. However, the FMA is rarely used in clinical settings and has been overall replaced by more streamlined measurements such as the NIH Stroke Scale (NIH SS), which provide very little biomechanical information. The transition away from the FMA to the NIH SS is in part due to the atmosphere of the clinical sphere, in which compensation and restitution are not approached separately. Any form of functional recovery may be helpful and should not be limited to what is believed to be the 'correct' pattern of limb coordination. 
Stroke and the Corticospinal Tract

The corticospinal tract (CST) is the primary motor tract controlling the hand in healthy individuals (Lawrence and Kuypers 1968). The CST originates in the cortex and projects to the spinal cord. It is lateralized, so that the activity of one side of the body is controlled by CST projections from the contralateral heimsphere. Damage to the primary motor cortex, or a lesion to the descending tract from the cortex to the spinal cord (the CST) can cause a loss of voluntary control of hand movements (Lawrence and Kuypers 1968). The movements are weakened, slow, and individual fingers and joints lose the ability to be controlled independently of others. It is a normal for fingers $2-5$ to move together, so that attempting to move one finger causes the movement or force to be produced at other fingers with the exception for the thumb (Bourbonnais et al. 1989). This force enslaving is due to both biomechanical and neural linkages between these fingers. In order to move a finger independently, additional muscles are activated to prevent coupled fingers from moving, as seen in typing. However, when stroke involves the CST, the coupling between all fingers increases. Individuals become unable to move individual fingers without moving the others and can only do general opening and closing of all fingers with difficulty. Damage to CST also tends to result in the coupling of finger motion to the motion of the wrist, elbow, or even at the shoulder, as described above. This shows that the output of the motor cortex works to both activate muscles that are voluntarily activated, and to suppress unintended motion from other body parts that move either passively (biomechanic coupling through ligaments, or interaction torques across joints) or actively (muscles activated together).

Although CST damage accounts for muscle weakness, it does not directly cause joint coupling (Schulz et al. 2012). Therefore, other neuromechanical mechanisms are the likely cause of joint coupling. In the chronic stage of stroke, increased activity is seen in the contralesional cortex after stroke when a subject attempts to move their paretic limb. Because of this, the contralesional CST was once proposed to be the cause of the flexion synergy. However, there are significant problems with this theory. First, increased contralesional activity is often attributed to the post-stroke interhemispheric competition model. It is believed that in healthy individuals, the motor cortex of each hemisphere will directly inhibit the other to prevent mirror movement (Di Pino et al. 2014). In the post-stroke interhemispheric competition model, reduced motor cortical excitability in the damaged hemisphere reduces its inhibition of the contralesional cortex (Calautti et al. 2007; Di Pino et al. 2014). According to this model, it is difficult to distinguish whether the increased activity of the contralesional cortex is an adaptive change that 
accompanies regained function, or maladaptive plasticity, or simply a consequence of a loss of normal inhibition that does not result in changes in motor function. Second, the increased activity in the contralesional cortex could also be a combination of a greater reliance on the nonparetic limb associated with a high prevalence of learned non-use (Kerr et al. 2011). The idea that the contralesional CST causes the flexion synergy was largely put to rest when it was seen that input from the contralesional CST onto hand muscles of the affected side of the body is very weak (Baker et al. 2015) and does not favor flexor activity over extensor activity (Zaaimi et al. 2012).

Stroke and the Reticulospinal tract

The reticulospinal tract (RST) supports synergistic control of multiple motor actions through collaterals triggering the coordinated recruitment of multiple muscles (Davidson and Buford 2006). The RST is a brainstem pathway that innervates axial muscles and the proximal muscles of the extremities and is best known for its role in locomotion and postural control (Drew et al. 2004). In a lesser-known capacity, it projects bilaterally and also innervates distal muscles of the arm, including elbow, wrist, and hand muscles (Baker 2011; Davidson and Buford 2006). The RST originates from the pontomedullary reticular formation (PMRF) and projects bilaterally both onto interneurons and directly onto motor neurons (Kuypers 1964; Matsuyama et al. 1997; Sakai et al. 2009). Cortical input to the PMRF originates bilaterally from primary, premotor, cingulate, and supplementary motor cortices (Keizer and Kuypers 1989). Projections from the PMRF to the spinal cord are mainly contralateral, but with some ipsilateral projections onto the most proximal muscles. PMRF activity facilitates the activation of ipsilateral flexors and contralateral extensors, as well as simultaneous suppression of ipsilateral extensors and contralateral flexors (Davidson et al. 2007; Davidson and Buford 2004; Sakai et al. 2009).

There are two descending reticulospinal tracts with different origins and different functions. These provide balanced excitatory and inhibitory inputs to the spinal motoneurons and interneurons. The dorsal RST originates from the dorsolateral reticular formation in the medulla and receives facilitation from the motor cortex via corticoreticular fibers. The lateral CST and corticoreticulospinal tract descend adjacent to each other in the dorsolateral funiculus at the spinal level. The dorsal RST provides dominant inhibitory effects to spinal reflex circuits. In the context of stroke with cortical and internal capsular lesions, damage often occurs to both CST and corticoreticular tracts due to their anatomical proximity. The medial RST originates primarily from the pontine tegmentum with connections to PMRF. The medial RST descends along with 
the vestibulospinal tract (VST) in the ventromedial cord. Medial RST and VST provide excitatory inputs to spinal reflex circuits. The medial RST and VST tend to de damaged by stroke less often Hyperexcitability occurs when the dorsal RST is damaged (Bhadane et al. 2015; Brown 1994; Sheean 2002). Therefore, anatomical evidence suggests that the hyperactive stretch reflexes preferentially in flexor muscles that are the hallmark of stroke spasticity can be attributed to either a loss of inhibition from the dorsal RST or an increase in activity from the medial RST. However, physiological tests of this idea often have conflicting results (Li 2017; Li et al. 2017; Li and Francisco 2015).

In 2012, Stuart Baker's lab found substantial evidence attributing the source of flexion synergy in chronic stroke to the activity of the reticulospinal tract (Zaaimi et al. 2012). Following the primate work of Davidson \& Buford that showed that stimulation of the RST for the ipsilateral arm preferentially elicited activity in flexor muscles (Davidson and Buford 2006; Herbert et al. 2010), they sought to test the role of the RST in the post-stroke flexor synergy. They lesioned the corticospinal tract in monkeys to model a middle cerebral artery (MCA) stroke and observed the recovery from the acute stage to the chronic stage. They observed the classic switch from flaccid paralysis to regained function with joint coupling and a flexed posture due to increased elbow flexion and shoulder abduction torques, reflective of what occurs in humans during stroke motor recovery. Furthermore, they identified the ipsilateral RST as the generator of these hyperactive flexor torques on the affected side. This confirmed previous research that showed a greater proportion of flexor muscles compared to extensors and stimulation of these pathways preferentially elicits EMG activity in ipsilateral flexor muscles (Riddle et al. 2009). However, non-human primate models are not perfectly reflective of what happens in humans. After a complete CST lesion in monkeys, they still maintain the ability to functionally use their hand. They have persistent extensor weakness, but they can still support their weight clinging to a bar in what is called a 'power grip.' Humans cannot do this after stroke. This conservation of function in non-human primates is possibly the result of an evolutionarily more extensive innervation of the hand motoneurons by the reticulospinal or rubrospinal tracts (Lawrence and Kuypers 1968; Zaaimi et al. 2012). In adult humans, the rubrospinal tract has weaker, less extensive innervation of hand muscles.

Thanks to innovative new imaging methods, we are now able to examine these pathways in humans non-invasively. In humans, reticulospinal hyperexcitability is often associated with abnormal motor synergy and disordered motor control in chronic stroke survivors. In a diffusion 
tensor imaging study, RST reorganization and strengthening were significantly correlated with both motor impairments and the flexion synergy (Owen et al. 2017). Studies by the Dewald lab have repeatedly demonstrated the relationship between the appearance of the flexor synergy and the ipsilateral reticulospinal tract in humans (Lan et al. 2017; McPherson et al. 2018). Recently, they have used magnetic resonance imaging (MRI) and electroencephalography (EEG) to reveal a relationship between the increased activity in the ipsilateral corticoreticulospinal tract and the increased flexion synergy during grasp (Wilkins et al. 2020). Other research also supports the core idea that the joint coupling and spasticity is attributable to a combination of residual CST capacity and an upregulated RST. Taken together, there is substantial behavioral evidence in both animal models and humans to support the role of RST in the appearance of flexion synergy after stroke.

The presence of joint coupling also can be explained by the anatomy of the RST. Innervations from the tract onto alpha motor neurons of the spinal cord are highly branching, creating a 'low resolution' signal in which has fewer discrete ways to activate muscles independently (Davidson and Buford 2006). Movement is produced, but it is constrained to a stereotypical multi-joint pattern due to the activity in muscle spanning multiple joints. Another theory is that increased RST activity post-stroke increases the descending 'neural drive' to the paretic limb as spared ipsilesional motor resources become exhausted ( $\mathrm{Li}$ et al. 2019). This theory suggests that the RST does not participate directly in control, but rather increases the sensitivity of motoneurons to the residual CST inputs.

\section{Stroke and the Vestibulospinal Tract}

The vestibulospinal system also receives input from the somatosensory cortex and premotor cortex (Akbarian et al. 1994; Wilson et al. 1999), but is traditionally thought to play a role in activating antigravity muscles, and therefore not a good candidate to assign the cause of the flexion synergy. However, there are reasons to reconsider the importance of this pathway. Flexion synergies become extension synergies in monkeys when they are upside down, showing that vestibular input does affect their behavior (Denny-Brown et al. 1964). A thorough review by $\mathrm{McC}$ all et al. describes how reticulospinal neurons receive extensive inputs from the vestibular tract, and that these interactions are reflected in the changes to both reflexes and muscle tone (McCall et al. 2017). We have an incomplete understanding of the relationship between these tracts in humans, as the research that underlies our current understanding has been almost exclusively limited to animal studies. 
Stroke and the Supplementary Motor Area and Premotor Cortex

It was noted earlier in this review that increased activity of the contralateral motor cortex occurs during paretic arm movement after stroke, but that the contralesional CST does not have strong uncrossed projections to the paretic hand. However, increased activity also occurs in the contralesional Premotor Cortex (PMC) and Supplementary Motor Area (SMA). Furthermore, uncrossed indirect motor pathways originating from sensorimotor cortices have been shown to generate ipsilateral motor output (Baker et al. 2015) through corticobulbar projections. As stated earlier, direct cortical input into the reticular formation from the cortex comes ipsilaterally not only from the primary motor cortex, but also PMC and SMA (Chen et al. 2019). Using MRI and high-density EEG, Wilkins et al., (2020) found that long-term structural changes and increased activity in the contralesional (ipsilateral) PMC are associated with the flexion synergy. Investigating long-term structural changes in the ipsilateral cortex using MRI showed increased grey matter density in the SMC and PMC ipsilateral to the paretic arm of individuals with stroke, but without an increase in grey matter density in the primary ipsilateral motor cortex. In stroke individuals with the flexion synergy, they found increased activity in the ipsilateral PMC during hand opening concurrent with voluntary shoulder abduction, compared with hand opening in isolation. This was not seen in stroke subjects that displayed no flexion synergy. Their major claim from these findings is that the addition of shoulder abduction during attempted hand opening increased reliance on ipsilateral secondary motor areas, such as the SMA and PMC. There is further neuroanatomical support for the recovery of hand function correlating with increased structural connectivity of the SMA and PMC projections to the reticular formation in the brainstem and regulating activity in the RST (Herbert et al. 2010, 2015). They believe that this is a compensatory pathway to restore some residual movement, and that relying on the pathway is what causes long-term changes in these regions. There is a relationship between functional activity and both synaptogenesis and dendritic growth commonly seen in animal training models (Murphy and Corbett 2009).

Neuroplasticity after Stroke

Neuroplasticity is the modification of synapses and connections, which modifies its function. This occurs during maturation and with learning, although the mechanism of learning has many different theories and mechanisms. The school of thought that is referenced most often is Hebbian learning (Hebb 1949), in which a straightforward premise that is simplified today as the phrase "neurons that fire together, wire together". This proposes that neuronal routes that are 
used repeatedly will become strengthened through their use, and subsequent activation of some of these neurons will result in the firing of others that have been associated with it, resulting in cognitive associations that can be conscious or subconscious. However, neuroplasticity involves not only strengthening connections, but also weakening them. Mechanisms of neuroplasticity include neurogenesis, synaptogenesis, and glial support. In addition to the formation of new synapses, existing synapses can be strengthened or weakened. Long Term Potentiation, or LTP, is the increased effectiveness, or "strength" or the synapse, which might be the result of a presynaptic modification, postsynaptic modification, or (likely) both. One mechanism of LTP is that the amount of neurotransmitter that is released in a synapse is increased, increasing the number of NMDA receptor activations on the post-synaptic cell, which in turn promotes synthesis of AMPA-R for the post-synaptic cell's dendrites and makes that cell more sensitive to neurotransmitters. Long Term Depression, or LTD, is the opposite effect, making the postsynaptic cell less apt to respond to a presynaptic action potential, and is caused by a decrease in calcium.

On a cellular level, astrocytes, oligodendrocytes, and microglia interact with neurons to play a role in neurogenesis and neuroplasticity (Amaral et al. 2013; Morice et al. 2011). Microglia and astrocytes release and re-uptake factors that have direct and indirect effects on neurons: ATP promotes heterosynaptic depression, Glutamate promotes synaptic degeneration and neurotoxicity, D-serene promotes excitotoxicity and LTP, Nitric oxide promotes LTP and longterm pre-synaptic facilitation, TNF-alpha responds to neuronal injury, and others.

Oligodendrocytes are directly involved in signal conduction, prevent axon cross-talk, and offer support and stability, which improves signal transduction and enhances the opportunity for LTP mechanisms. Astrocytes and oligodendrocytes have also been recently shown to play a role in energy metabolism through glucose uptake from blood and conversion to lactate and other products that the neurons can use, which important in both synaptogenesis and synaptic plasticity (Achour and Pascual 2010).

\section{Summary}

When motor activity returns during stroke recovery, it often has stereotypical coupled movement across multiple joints. One example of this joint coupling, the upper limb flexion synergy, directly impairs movement through the involuntary activation of flexor muscles for the elbow, wrist, and hand when abducting the shoulder. Many recent studies have supported an increased influence of corticobulbar, reticulospinal, and vestibulospinal activity in joint coupling after stroke. The 
appearance of joint coupling is thought to be due to the high level of branching in these pathways, leading to less specific activation of muscle groups than the corticospinal tract. How these pathways are affected by CST damage is unknown. Increased influence of these pathways could be due to increased dependency neuroplastic changes due to increased dependency on them during stroke recovery, but it could also simply be a consequence of a loss of inhibition from damaged cortical areas. Overall, understanding the mechanism and function of these pathways in stroke recovery may allow for the development of targeted neuronal treatment to improve stroke outcomes. Specifically, in Aim 2 I focused on the principle of joint coupling, and applied gravitational support (developed based on findings in Aim 1) to the shoulder in an attempt to improve hand performance after stroke.

\section{Part II: Stroke Assessments}

Clinical Assessments

The clinical assessment of movement deficits is a primary source of actionable information used to design a personal rehabilitation plan (Sullivan et al. 2013). Therefore, the quality and type of information obtained from the assessment dramatically influences the effectiveness of therapeutic intervention. Clinical tests are usually developed and validated to obtain a specific type of information about the quality of movement. For example, the Fugl-Meyer assessment (FMA) is designed to measure the level of impairment by quantifying a patient's ability to control independently individual joints (Fugl-Meyer et al. 1975). Other tests, such as Wolf Motor Function Test (WMFT) and the Action Research Arm Test (ARAT), focus on measuring the ability to perform functional tasks (Wolf et al. 2001a; Yozbatiran et al. 2008). These subjective tests have variable responsiveness and predictive validity for detecting effects of therapy for stroke and ceiling effects in patients with mild motor deficits (van der Lee et al. 2001a). More importantly, recent randomized clinical trials show variable effectiveness of treatments developed based on the evidence provided by these clinical assessments (Duncan et al. 2003a; Saposnik et al. 2016b; Wolf et al. 2010a). It is widely believed that higher quality evidence for informing the design of future interventions is needed (Krakauer and Carmichael 2017a; Pollock et al. 2014a).

\section{Motion Capture Assessments}

Motion capture assessments offer an objective way to assess movement deficits without requiring the presence of a clinician. Motion capture analysis has been shown to be more sensitive at distinguishing stroke impairment than clinical tests (Schwarz et al. 2019). It allows 
the discernment of compensatory movement strategies in contrast to the recovery of original movement coordination (restitution), which clinical tests cannot clearly decipher. Motion capture systems suitable for home use have already been used to quantify movement deficits after stroke and shown to be directly relatable to existing clinical assessments (Chang et al. 2011; Clark et al. 2013; Mousavi Hondori and Khademi 2014; Olesh et al. 2014). These measuring kinematic variables, such as movement extent, range of motion, accuracy, and speed. These are often given in game format and can be used as both a rehabilitation training tool and to assess movement improvement over time (Cannell et al. 2018). Although motion capture analysis after stroke continues to be incredibly diverse and requires standardization (Schwarz et al. 2019), these are incredible tools for both research and patient care.

The 2019 Stroke Recovery and Rehabilitation Roundtable recommended post-stroke movement analysis to include motion capture of planar 2-D reaching tasks with gravitational support and an unsupported 3-D functional task (Kwakkel et al. 2019). Kinematics should be taken from the trunk, arm, and endpoint (hand), with 2-D reaching endpoint and angular kinematics characterized along with endpoint accuracy and peak velocity. Within each movement and averaged across movements, a 'difference score' can be calculated to compare each subject to a non-disabled reference. The recommended 3-D functional task, drinking a glass of water (Alt Murphy et al. 2018) allows for natural, unrestrained movement of multiple joints to be analyzed in terms of joint kinematics. 3-D pointing tasks can be used to evaluate these same properties, however they must be planned to elicit movement across multiple joints simultaneously in order to accurately evaluate inter-joint coordination (Schwarz et al. 2019). Joint kinematics are analyzed either using dimensionality reduction to compare a reduced overall trajectory between subjects, or at the level of each joint degree-of-freedom (Shoulder flexion/extension, abduction/adduction, for example). By evaluating joint degrees of freedom independently, researchers can identify compensatory strategies. For example, this method found that in this task, increased trunk movement and shoulder abduction helped to compensate for reduced elbow mobility (Alt Murphy et al. 2018). These compensatory strategies may be contributing to a lack of fluidity in the movement. Using this type of analysis, researchers see that stroke patients have problems with movements that require inter-joint coordination and can begin to target rehabilitation methods to specific joints and design a more personalized treatment plan.

\section{Biomechanical Modeling}

Most of the studies that quantify motor deficits and rehabilitation use either kinematic measures of stability, range of motion, speed, and error, or strength-based measures of individual muscle 
groups (Gowland et al. 1993). Biomechanical analysis offers an alternative strategy to investigate impairment and neural mechanisms that result from stroke. In this, movement is defined through the identification of a joint location and an axis of movement of the joint, which compose a degree of freedom (DOF). The overall movement of the arm is caused by rotational forces (also called moments or torques) acting around each DOF. These net torques are a combination of passive and active torques. An example of passive torques are gravitational torques (the force gravity exerts on each limb segment) and interaction torques (movement interactions between body segments), An example of active torques are muscle torques (provided by muscle contraction). Kinematic analysis alone is incapable of distinguishing the differences between these sources of torque. While there exist techniques for obtaining dynamic measurements of individual muscle strengths without biomechanical modelling, most torque measurement techniques physically constrain the movement of the limb to reduce the number of degrees of freedom in their measurements. This includes using a robotic exoskeleton, a moveable platform, or a joystick. This limits the ability to capture natural movement during functional tasks, most notably reach and grasp.

In a biomechanical simulation, the active muscle torque that is necessary for a limb to go through movements requires it to 1) overcome inertia 2) stabilize the limb against simulated gravity, 3) stabilize against perturbation from interaction torques due to movement along the linkage of the arm. This is similar to that in which our body performs, however, is easier to compute. It is important to remember that while all of our natural movement is controlled directly by the activity of our muscles, there is no direct linear mapping from muscle contraction to movement. Rather, the system is incredibly complicated with many muscles acting on one or multiple joints. This is called an over-actuation problem. In addition, the contraction affects the joint torque in a way that is dependent on the distance of the body of that muscle from the center of the joint, a moment arm that changes throughout movement (Eq. 1). The effect muscle activity has on movement becomes more complicated as moment arms are dynamic and change as limb positions change.

Applications of Biomechanical Modelling

Although biomechanical assessments are found throughout the research literature, they have rarely been applied in clinical settings. Biomechanical assessments might produce measurements more directly related to neural control, and their integration may be key to 
understanding individual differences in stroke disability. Investigating movement in terms of biomechanics allows the unique advantage of being able to separate components of musclegenerated forces into those dependent and independent of gravity. Modelling allows us to identify profiles of active muscle torque that correspond to supporting the weight of the limb against gravity throughout a movement (Olesh et al. 2017). As described in Olesh et al. 2017, active muscle torque can be separated into two components, termed postural and dynamic components. The postural torque component captures the portion of muscle torque that supports the limb segments against the force of gravity; it can also be thought of as the postural component of forces produced by muscles. We refer to it as postural forces. The dynamic torque component represents the residual muscle torque related to motion production. We refer to it as dynamic forces. This component can also be through of as the muscle force that would produce the same motion without gravity, e.g. in a microgravity environment. To obtain the dynamic torque components, inverse dynamics simulations are first run with gravity of the physics engine set to zero. To obtain the postural component, the dynamic torque component was subtracted from the overall active muscle torque obtained from simulations with standard gravity for each DOF.

$$
\begin{aligned}
\tau_{M}=\sum_{i=1}^{N} \vec{R}_{i} \times \vec{A}_{i} & =\sum_{i=1}^{N} \vec{R}_{i} \times \vec{D}_{i}+\sum_{i=1}^{N} \vec{R}_{i} \times \vec{G}_{i} \\
\tau_{M} & =\tau_{M D}+\tau_{M G}
\end{aligned}
$$

Eq 1. Dynamic and Gravitational Muscle Torques. Muscles produce a tendon force (Ai) that, when multiplied by the distance that the muscle spans from the joint itself (moment arm, Ri), provides the actual torque on joint. Therefore, the active muscle torque (Tm) is equal to the sum of all of the activations of the muscles at that joint multiplied by their respective moment arms. This is theoretically equal to active muscle torques obtained with inverse simulations, which can be further subdivided into dynamic (TMD) and gravitational (TMG) support torque components. Substituting into the original formula, it could be suggested that the muscle activation required used for dynamic movements (Di) and the activations for postural support (Gi) are separate systems that sum to produce the overall torque.

Quantifying the gravity-related component of muscle torque may provide valuable insights into the post-stroke disruption of postural mechanisms of movement control. As we know, gravity plays an important role in movement control contributing to both posture and movement 
(Lackner and DiZio 2000; Pozzo et al. 1998). These gravity-compensating components of muscle activity may also be driven by the reticulospinal pathway involved in anticipatory postural adjustments (Schepens et al. 2008; Schepens and Drew 2004, 2006). The reticulospinal pathway has been implicated in post-stroke unmasking of flexor synergies involving the antigravity muscles of the arm that are a major contributor to motor deficits (Ellis et al. 2007, 2012). Second, this technique may offer the key feature necessary for direct comparisons between unassisted, free movement and movement performed while physically removing the necessary forces needed to lift the arm against gravity. As discussed previously, it is recommended that a gravity support device be used to standardize planar reaching tasks. The structure of these assessments makes them highly restricted and useful to make comparisons between different studies. However, the reliance on a gravity-assistive device for radial reaching tasks makes it difficult to directly apply this analysis to movements captured using at-home motion capture systems. The computational ability to remove the forces generated to resist gravity may be an ideal strategy to allow for assisted radial reaches to be compared.

Additionally, the prediction of the necessary muscular forces for gravity resistance may aid in future development of assistive technology. Unloading the arm against gravity in stroke survivors with hemiparesis has been shown to improve reach distance and grasping performance (Ellis et al. 2009; Sanchez et al. 2006; Sukal et al. 2007), while additional loading at the shoulder worsens performance with hemiparetic hand (Lan et al. 2017). Profiles of gravity-resisting torque could be given in the form of assistive Functional Electrical Stimulation (FES), which has low-cost and wearable applications. This application will be tested in Chapter 3.

\section{Summary}

High-quality, individualized descriptions of post-stroke motor deficits can be obtained using motion capture and inverse dynamics of stereotypical reaching movements. It is also possible that muscle torques, estimated through equations of motion, can provide a more accurate representation of neural control compared to angular kinematics. Furthermore, analysis separates muscle torques into components related to gravity compensation and motion production may provide a deeper understanding of the neural mechanisms involved in motor control. The ability of metrics to classify stroke impairment can be compared against angular kinematic measures will be discussed in chapter 2 . We believe that isolating gravity-dependent and gravity-independent components of muscle torque can provide unique neurophysiological 
information about post-stroke motor deficits, assist with assessment, and improve rehabilitation strategies.

\section{Part III: Personalized rehabilitative techniques and assistive devices}

Rehabilitative Therapy

Cortical damage from a stroke often disrupts descending motor and sensory signals and impairs sensorimotor integration. Unilateral upper limb motor deficit is the most common motor symptom after stroke, with function directly affected by motor and sensory impairments. This produces errors in accuracy and reliability of movement and poor grasping function, which also contribute to affected arm disuse after stroke. Lack of use of the limb exacerbates motor decline, but there are many patients with severe motor impairment that do not have enough control of the limb to use it reliably outside of the clinic, which limits the progress made with physical therapy. Rehabilitation programs for these chronic motor-impaired patients would benefit from the ability to be performed at home with minimal human intervention. Constraintinduced movement therapy (CIMT), is in the spotlight currently as an excellent at-home option with significant impact made in the chronic stage. In this technique, an individual is physically prevented from using their more mobile limb to force them to use their impaired one, using a mitt or a sling. However, severe motor impairments can exclude patients from this treatment option because of the risk of falling, the psychological effects of limb restriction in patients with cognitive depression or helplessness, or the patient simply not having enough movement in the impaired arm to perform daily tasks. Inclusion criteria for CIMT includes the ability to voluntary extend their fingers and wrist at least 10 degrees and have enough mobility to keep their functional hand restrained for hours, which prevents many stroke patients from benefiting from the technique. It would be valuable to pursue alternative therapeutic approaches for these patients that can facilitate the use of the paretic upper extremity to facilitate a functional recovery.

An alternative to CIMT is FES, which can be used to recover functionality in paralyzed or dysfunctional limbs or as a corrective neuroprostheses. Various wearable neuroprosthetics for grasp restoration using FES have demonstrated rehabilitative success in acute and chronic stroke patients (Micera et al. 2010). FES generates action potentials in the muscle fibers and motor units that innervate muscle fibers, resulting in contraction of the muscle (Koutsou et al. 2016). Most applications target the motor neurons entering the muscle, and not the muscle fibers themselves, to produce a functional contraction. This is because excitation thresholds are 
significantly higher in muscle fibers (>1003) compared with motor neurons and can drive muscle activity using less electrical charge. FES can be delivered through different interfaces such as implanted electrodes (epineural or intramuscular electrodes) or transcutaneous electrodes. Transcutaneous FES is noninvasive and uses surface electrodes on the skin, often in a wearable device. Transcutaneous FES is delivered by a burst of short electrical charge pulses applied between pairs of electrodes positioned on the skin. However, they provide selective activation only for muscles close to the surface. Despite this limitation, transcutaneous FES of superficial muscles is seen to be sufficient for inducing reaching and grasping (Reviewed in Micera et al., 2010). Evidence suggests that the therapeutic effect of the treatment occurs through a similar mechanism as that of repeated task practice. In cases of patients that cannot perform the task that they need to practice, wearable transcutaneous FES may provide the solution. For the rest of this thesis, all FES referred to will be transcutaneous FES.

\section{Clinical use of Functional Electrical Stimulation}

FES in laboratory settings have shown excellent performance; however, FES devices are being used significantly less often by clinicians than cyclic neuromusclular electrical stimulation (NMES). Cyclic NMES is a similar technique that electrically activates muscles rhythmically but is not used for functional control of an intentional movement in the upper limbs as these behaviors are not cyclic in nature. Cyclic NMES has also been seen to promote distal motor recovery in chronic impaired stroke survivors for the lower limbs (You et al. 2014). FES is shown to have similar rehabilitative effects as cyclic NMES in upper limbs in research settings (Knutson et al., 2016) and has the ability to restore function immediately for daily tasks but is a less attractive option for clinicians because of three major factors: 1) High cost of instruments, 2) Lack of user-friendliness, and 3) Poor performance in the clinic due to misalignment of instruments.

The results of clinical trials for the long-term improvement of reach and grasp through FES often has inconclusive results (Langhorne et al., 2009, Pelton et al., 2012, Vafadar et al., 2015). A 2009 meta-analysis of 13 clinical trials (331 recruited, 277 analyzed) by Langhorne et al. found that there is insufficient data on the effectiveness of transcutaneous FES for hand function rehabilitation (Langhorne et al., 2009). In a 2012 literature review by Pelton et al., it was again stated that there was not enough sufficient research to show the efficacy of FES in reaching and grasping rehabilitation after stroke. The limitations of FES outlined by Pelton included 1) insufficient selective activation of muscles, and 2) muscle fatigue as a reaction to muscle 
stimulation. These limitations have been directly targeted through improvements on control strategies. Improved, user friendly control strategies for FES application is imperative for the clinical translation of FES.

\section{FES Mechanism}

Whether using an assistive robotic or stimulation device long-term is as effective in restoring non-assisted movement as therapeutic treatments is a matter of debate in the field. However, as long as the assistive device encourages efficient muscle coordination for movement, it follows the same fundamental mechanism as direct human manipulation of the limb or a targeted training regimen, or electrical stimulation of weak muscles, which have all been shown effective in clinical use (Pollock et al. 2014a). One difference is that there are differences in the way that muscles are recruited in volitional movement compared to FES-elicited movement. During volitional movement, motor neurons asynchronously activate the muscle fibers. This asynchronous activation is temporally organized in a way that allows fine control of contraction (and muscle force) from low to tetanic level. However, the mechanism of FES-generated contraction does not follow this activation pattern intrinsically. In FES, the generated electric field activates the fibers synchronously, which does not allow the same level of fine control of contraction and increases the rate of fatigue (Bigland-Ritchie et al. 1979). The negative effect of muscle fatigue induced by FES is temporary but should be improved to maintain the safety and practicality of the device. One goal in FES advancement is to design systems that provide asynchronous activation and mimic the natural behavior of CNS descending signals.

Techniques for the alleviation of muscle fatigue during electrical stimulation include improving electrical stimulator control parameters, often through closed-loop systems, and improving upon the design and placement of FES electrodes. These techniques have been shown to demonstrate the delay of muscle fatigue, and the combination of these improvements may greatly improve the efficacy.

\section{FES and Neuroplasticity}

FES can induce synaptic plasticity based on the principles of spike timing-dependent plasticity (STDP) (Foysal et al., 2016, Baker 2021). Stimuli exciting a presynaptic neuron within the motor control pathway, presented quickly in time immediately preceding the excitement of a postsynaptic neuron, with strengthen the connection between the two cells, an effect known as longterm potentiation (LTP) (Markram et al., 1997). This can be performed on a cell with multiple 
inputs by selectively stimulating two of the inputs onto the postsynaptic cell with a time delay and can be performed non-invasively by stimulating upsteam sensory pathways (Stefan et al., 2000; Ridding and Uy, 2003). FES directly activates lower motor neurons and associated sensory systems. Activity in these systems are known to induce cortical synaptic plasticity, causing changes in cortical activity (Quandt and Hummel 2014). Baker lab 2016 developed a wearable device to induce synaptic plasticity using biceps activation using a paired auditory stimulus, which lead to plastic changes in the stretch reflex, this device improved upper limb function after stroke in a small set of stroke subjects (Choudhury et al. 2020). This effect was attributed to a strengthening of the connections within the reticulospinal tract using non-invasive probe techniques of TMS, CMET and examining the StartReact effect, a protocol developed to examine the contribution of reticulospinal activity to movement by comparing the reaction timing of responding to a quiet auditory cue versus a loud, startling sound with a preplanned movement. This technique works as the RST is thought to have a greater contribution to movement elicited by the startle reflex than voluntary movement in non-startling conditions. While auditory stimulation of cells in the RST promotes synaptic plasticity in these experiments, fitting the stimulation parameters to the specific intention of the user's movement can also induce beneficial synaptic plasticity. This is a fundamental concept in the application of rehabilitative FES.

\section{Conclusions}

Functional Electrical Stimulation (FES) is currently in use as a therapeutic treatment for improving reaching and grasping, but poor clinical results have suggested the need to improve the translation of the device from the lab into the clinic. This will primarily include improving FES control strategies, increasing the user-friendliness of the device for patients and clinicians, and keeping the price of treatment reasonable. FES can be used assistively, to grant the user the return of lost function and help them gain their independence. It may also induce neuroplastic changes that further improve motor ability. With recent technology and the push towards personalized care, there is a lot of potential for FES. Using an assistive robotic device to passively reduce the load of gravity on the shoulder during reaching and grasping has also been shown to improve muscle coordination for grasp, but functional electrical stimulation (FES) for gravity support of shoulder muscles has not yet been tested. This approach is more affordable than robotic systems, and may have additional rehabilitative benefits, such as improved muscle tone. Assistive devices, including FES, can boost activity to increase mobility and prevent non- 
use, and based on principles of neuroplasticity, an increase in use may lead to long term recovery. 


\section{Chapter 2) Stroke Movement Analysis}

This chapter has been adapted from Thomas et al (2021).

Title: Muscle torques and joint accelerations provide more sensitive measures of post-stroke movement deficits than joint angles.

Authors: Ariel B. Thomas ${ }^{1,2}$, Erienne V. Olesh ${ }^{1,2}$, Amelia Adcock $^{3,4}$, and Valeriya Gritsenko ${ }^{1,2}$

Affiliations:

${ }^{1}$ Department of Human Performance, Division of Physical Therapy, School of Medicine West Virginia University, Morgantown, WV, 26506

${ }^{2}$ Rockefeller Neuroscience Institute, Department of Neuroscience, West Virginia University, Morgantown, WV, 26506

${ }^{3}$ WVU Center for Teleneurology and Telestroke, Morgantown, WV, 26506

${ }^{4}$ Department of Neurology, School of Medicine, West Virginia University, Morgantown, WV, 26506

Corresponding author: Valeriya Gritsenko, PO Box 9226, One Medical Center Drive, Morgantown, WV, 26505-9226. Phone: (304) 293-7719

Running Head: dynamic assessment of post-stroke reaching

Supplemental Figures are available at https://zenodo.org/record/5044555\#.YQQEpS1h3q0 


\section{Abstract}

The whole repertoire of complex human motion is enabled by forces applied by our muscles and controlled by the nervous system. The impact of stroke on the complex multi-joint motor control is difficult to quantify in a meaningful way that informs about the underlying deficit in the active motor control and intersegmental coordination. We tested whether post-stroke deficit can be quantified with high sensitivity using motion capture and inverse modeling of a broad range of reaching movements. Our hypothesis is that muscle moments estimated based on active joint torques provide a more sensitive measure of post-stroke motor deficits than joint angles. The motion of twenty-two participants was captured while performing reaching movements in a center-out task, presented in virtual reality. We used inverse dynamics analysis to derive active joint torques that were the result of muscle contractions, termed muscle torques, that caused the recorded multi-joint motion. We then applied a novel analysis to separate the component of muscle torque related to gravity compensation from that related to intersegmental dynamics. Our results show that muscle torques characterize individual reaching movements with higher information content than joint angles do. Moreover, muscle torques enable distinguishing the individual motor deficits caused by aging or stroke from the typical differences in reaching between healthy individuals. Similar results were obtained using metrics derived from joint accelerations. This novel quantitative assessment method may be used in conjunction with home-based gaming motion-capture technology for remote monitoring of motor deficits and inform the development of evidence-based robotic therapy interventions.

New and Noteworthy

Functional deficits seen in task performance have biomechanical underpinnings, seen only through the analysis of forces. Our study has shown that estimating muscle moments can quantify with high sensitivity post-stroke deficits in intersegmental coordination. An assessment developed based on this method could help quantify less observable deficits in mildly affected stroke patients. It may also bridge the gap between evidence from studies of constrained or robotically manipulated movements and research with functional and unconstrained movements.

Keywords: 3D Arm Movements, Stroke Paresis, Nonlinear Dynamics, Motor Control, Motor Assessment 


\section{Introduction}

Movement is a complex interplay between forces generated by our muscles under the control of the central nervous system and the environment. Neurological diseases such as stroke damage the neuromuscular mechanisms of movement production. The resulting movement deficits are a major contributing factor to stroke being the leading cause of long-term disability in the United States (Benjamin et al. 2019). The clinical assessment of movement deficits is based on the observation of movements by experts. For example, sophisticated clinical tests such as the Fugl-Meyer Test of impairment (Fugl-Meyer et al. 1974) or the Wolf Motor Function Test and the Action Research Arm tests of functional ability (van der Lee et al. $2001 \mathrm{~b}$; Wolf et al. 2001b) rely on expert's timing and rating the quality of several observed movements, some of which are focused on moving single joints while others on complex functional tasks. However, these tests cannot account for the redundancy of the arm anatomy that underlies inter-subject variability, i.e. the typical differences in how movements are produced by individuals. For example, the same endpoint movement can be performed with different combinations of peak shoulder flexion and abduction angles, i.e., with more or less elbow elevation, which in turn affects the elbow angle. The challenge for motor assessment is in distinguishing the typical variability due to this redundancy from the abnormal changes in movement caused by stroke. Furthermore, the prevalence of stroke increases with age (Virani Salim S. et al. 2021), so that sarcopenia and age-related neurological changes further contribute to the individual differences in how movements are produced (Dutta and Hadley 1995; Edström et al. 2007; Evans 1995; Evans and Campbell 1993; Seidler et al. 2010; Shaffer and Harrison 2007). To compensate for this individual variability in how movements are generated, clinical tests typically employ a low-resolution scoring system and extensive training of raters to maximize inter-rater validity. However, this leads to reduced responsiveness and predictive validity of clinical tests and ceiling effects in patients with mild motor deficits (Hsieh et al. 2009; van der Lee et al. 2001b). Importantly, randomized clinical trials show variable effectiveness of treatments developed based on the evidence provided by these clinical assessments (Duncan et al. 2003b; Saposnik et al. 2016a; Wolf et al. 2010b). It is widely believed that higher quality assessment measures for informing the design of future interventions are needed (Krakauer and Carmichael 2017b; Pollock et al. 2014b).

Motion capture offers an objective way to assess movement deficits using algorithms (Schwarz et al. 2019). However, multijoint motion is complex and multiple variables derived from motion capture can describe it. Here we focus on angular variables, such as joint angles, angular velocity, and joint torques, instead of linear variables, such as linear endpoint or joint 
kinematics, linear accelerations, or linear forces. This is because the former is in the intrinsic coordinate system of the neuromuscular system, while the latter is in the extrinsic world coordinate system. Measures derived from motion capture, such as the temporal profiles of joint angle changes during movements, are directly relatable to existing clinical assessments (Chang et al. 2011; Clark et al. 2013; Mousavi Hondori and Khademi 2014; Olesh et al. 2014). Metrics that capture inter-subject and inter-trial variability are preferred as they help to standardize motor assessment based on motion capture (Schwarz et al. 2019). In our study, we were inspired by these recommendations and used the performance index, a measure that was originally developed by Fitts to quantify the information-transmission capacity of the motor system (Fitts 1954). The performance index quantifies the information content of movement trajectories based on amplitude, accuracy, and timing of movement, combining the different variables that contribute to inter-subject and inter-trial variability. Because these parameters are interrelated, a relationship often referred to as speed-accuracy tradeoff. Analyzing them independently does not provide a holistic picture of the changes in performance that were caused by the changes in each of these parameters. Here we have used the performance index to control for the speed-accuracy tradeoff of individual participants reaching with different velocities and measure the changes in the information-transmission capacity that are independent of that tradeoff.

The 2019 Stroke Recovery and Rehabilitation Roundtable recommended post-stroke movement analysis to include motion capture of planar (2D) reaching tasks with gravitational support and unsupported three-dimensional (3D) functional tasks (Kwakkel et al. 2019). The functional tasks were recommended specifically to enable quantifying intersegmental or intersegmental coordination using the analysis of natural, unrestrained, and simultaneous movement of multiple joints (Schwarz et al. 2019). Moreover, the analysis of joint angles as independent degrees of freedom (DOFs) enables the identification of compensatory strategies. For example, increased shoulder abduction helps to compensate for reduced elbow mobility (Murphy et al. 2011). In our study, we applied similar analyses to study intersegmental coordination in several people with chronic stroke and compare it to the intersegmental coordination in young and aged controls. We have directly compared the temporal profiles of kinematic and dynamic variables between limbs using the coefficient of determination. This metric derived from joint angles is closely related to the qualitative scores of Fugl-Meyer Assessment (Olesh et al. 2014). However, measuring kinematic variables, such as joint angles, can provide only indirect information about how the movement is generated by neuromuscular action. It is not possible to infer from kinematic measures what forces were applied by the 
muscles to make the arm move, even for a seemingly simple but clinically important determination of whether the motion is active or passive. The forces muscles produce can be estimated by inverse modeling using the equations of motion. The temporal profiles of joint angles obtained from motion capture are differentiated, combined with the estimates of segment inertia, and plugged into the equations of motion to compute net, passive, and active or applied joint torques (Ellis et al. 2009; Russo et al. 2014; Sainburg et al. 1995; Shabbott and Sainburg 2008). This inverse modeling approach takes into account passive torques caused by complex limb inertia and extrinsic forces, such as gravity or objects held in the hand. The active joint torques, termed muscle torques, are the summed result of all the moments generated by muscle contractions representing only the applied forces necessary to produce the observed motion in the presence of all the internal and external passive forces (Dounskaia and Wang 2014; Gentili et al. 2007; Le Seac'h and McIntyre 2007; Papaxanthis et al. 2005; Wang and Dounskaia 2016). Muscle torques can then be used to quantify the post-stroke changes in the production of active movement while controlling for the role of passive forces. Moreover, muscle torques can be related directly to the forces produced by muscle contractions through moment arms. Therefore, the analysis of muscle torques enables us to approximate the typical wholelimb patterns of neural motor commands during reaching and to quantify changes in those patterns after stroke in terms that are relevant to the neural mechanisms of motor control. Here we used both the performance index and the coefficient of determination derived from joint angle and muscle torque trajectories to compare their relative information content and gain insight into the potential of these metrics to assess age-related and post-stroke motor deficits.

The component of muscle torque that is responsible for intersegmental coordination can be extracted computationally. The inverse dynamic simulations used to estimate muscle torque can be run without gravity, simulating the active torques necessary to produce the observed motion without gravity (Olesh et al. 2017). This dynamic component of muscle torque also reflects the forces that are produced during planar reaches with gravitational support. Such 2D movements are used for quantifying post-stroke motor deficits and robot-assisted rehabilitation (Coderre et al. 2010; Keeling et al. 2021; Kwakkel et al. 2019; Scott and Norman 2003). Studies of multisegmented arm motion with and without gravity have shown that this dynamic component of muscle torque reflects intersegmental coordination (Debicki and Gribble 2005; Gribble and Ostry 1999; Ketcham et al. 2004; Pigeon et al. 2003). Deficits in intersegmental coordination have been reported in people with cerebellar strokes (Bastian et al. 1996). The dynamic component of muscle torque is thought to be related to the phasic component of muscle activity, which is the remainder after subtraction of the tonic posture-related component 
of muscle activity (Olesh et al. 2017). Moreover, it has been shown that cerebellar damage preferentially impairs phasic EMG (Manto and Bosse 2003). Therefore, quantifying the dynamic component of muscle torque may provide a method to assess post-stroke disruption in intersegmental coordination.

Our study aim was to quantify objectively post-stroke motor deficits using motion capture and inverse dynamics of stereotypical reaching movements. Here we define a motor deficit narrowly as a statistical difference in individual metric derived from kinematic or dynamic variable of movement from the mean metric for young controls. Our hypothesis is that muscle torques contain more information about post-stroke motor deficits than angular kinematics. Because of the small sample size and the heterogeneity of our sample, we designed our experiment to quantify individual motor deficits in participants with stroke relative to aged individuals and the group mean of young controls. We then applied a novel analysis that separated muscle torques into components related to gravity compensation and intersegmental coordination and used these and kinematic measures to quantify motor deficits.

Methods

Participants

Twenty-two human participants were recruited to perform reaching movements to virtual targets with both arms. The participants were divided into three groups, Control, Stroke, and Aged. The Control group ( $23 \pm 1.2$ years) included 9 participants without any known neurological or musculoskeletal disorders. The kinematic and torque data from the Control group were reported in Olesh et al. (35). The Stroke group included 8 participants (58 \pm 6.9 years), who have suffered single unilateral ischemic stroke at least three months prior to the experiment (Table 1). Diagnosis was performed by neurologists during hospital admission or following neurological evaluations at Ruby Memorial Hospital. Individuals were excluded if they could not produce visible movement with their shoulder and elbow, or if they were unable to provide written consent to participate. The Aged group (58 \pm 2.4 years) included 5 participants without any known neurological or musculoskeletal disorders, whose age was within the mean \pm SD of participants in the Stroke group. All control participants were right-hand dominant and reported no unrelated movement disorders or significant injuries to their upper extremities. The study and the consent procedure were approved by the Institutional Review Board of West Virginia University (Protocol \# 1311129283). All participants provided written consent before participation. 
Table 1. Characteristics of individuals with stroke.

\begin{tabular}{|c|c|c|c|c|c|c|c|}
\hline ID & Gender & $\begin{array}{l}\text { Infarct } \\
\text { hemi- } \\
\text { sphere }\end{array}$ & $\begin{array}{l}\text { Infarct description and } \\
\text { location }\end{array}$ & $\begin{array}{l}\text { Age } \\
\text { (years) }\end{array}$ & $\begin{array}{l}\text { Period } \\
\text { post- } \\
\text { stroke } \\
\text { (years) }\end{array}$ & $\begin{array}{l}\text { Contra- } \\
\text { lesional } \\
\text { reach } \\
\text { duration (s) }\end{array}$ & $\begin{array}{l}\text { Contra- } \\
\text { lesional } \\
\text { endpoint } \\
\text { accuracy }(\mathrm{cm})\end{array}$ \\
\hline $\mathrm{S} 1$ & $\mathrm{~F}$ & Left & Left MCA & 51 & 3 & $0.7 \pm 0.16^{*}$ & $4.5 \pm 0.87$ \\
\hline $\mathrm{S} 2$ & M & Left & $\begin{array}{l}\text { Left caudate lenticular } \\
\text { nucleus and external } \\
\text { horn of the left } \\
\text { ventricle }\end{array}$ & 58 & 5 & $0.8 \pm 0.11^{*}$ & $4.1 \pm 1.22$ \\
\hline S3 & M & Right & $\begin{array}{l}\text { Right dorsal pontine- } \\
\text { medullary lacunar } \\
\text { infarction }\end{array}$ & 67 & 0.5 & $0.7 \pm 0.17^{*}$ & $5.6 \pm 1.37$ \\
\hline S4 & M & Right & $\begin{array}{l}\text { Lacunar infarct in } \\
\text { posterior right } \\
\text { putamen and border of } \\
\text { right internal capsule }\end{array}$ & 68 & 0.25 & $0.5 \pm 0.08$ & $4.5 \pm 1.04$ \\
\hline S5 & M & Left & Left MCA & 53 & 0.5 & $1.3 \pm 0.52 *$ & $8.2 \pm 2.80$ \\
\hline S6 & M & Right & Right MCA & 60 & 6 & $0.5 \pm 0.10$ & $4.3 \pm 1.37$ \\
\hline S7 & M & Right & $\begin{array}{l}\text { Right lateral } \\
\text { medullary infarction } \\
\text { with occluded right } \\
\text { vertebral artery }\end{array}$ & 51 & 8 & $1.4 \pm 0.49 *$ & $8.6 \pm 2.63$ \\
\hline S8 & M & Right & $\begin{array}{l}\text { Right MCA, extending } \\
\text { posteriorly }\end{array}$ & 53 & 7 & $0.4 \pm 0.12$ & $7.2 \pm 3.30$ \\
\hline
\end{tabular}

MCA stands for middle cerebral artery. Standard deviations are across movement directions. * indicates significant difference from Control group with $p<0.0031$ for all; Significant alpha with Bonferroni correction for repeated measures $=0.0031$. Note, that the amplitude of movement in S8 participant was decreased due to his inability to reach the same distance as controls.

Experimental Task

During the experiment, participants reached to virtual targets in a center-out task, as described in detail in Olesh et al. (35) (Fig. 1). The arm was not supported, all movements were unconstrained. Movements were instructed using a virtual reality (VR) software (Vizard by Worldviz) and headset (Oculus Rift), which randomly displayed one of 14 targets, $10 \mathrm{~cm}$ in diameter, arranged equidistantly from a center target. A center target was placed in the VR space so that the initial posture of the upper arm was aligned with the trunk (all shoulder angles 
at $0 \circ$ ) and the forearm parallel to the floor palm down (elbow angle at $90 \circ$ and wrist at $0 \circ$; Fig. $1 \mathrm{~A})$. This minimized both the intertrial variability and inter-subject variability in motion trajectories. For each participant, the distance from the center target to the peripheral targets was scaled to $30 \%$ of arm length (anterior acromial point to the distal tip of the index finger). This distance between the central and peripheral targets was on average $20 \mathrm{~cm}$. One most impaired participant (S8, Table 1) was unable to reach targets reliably with his contralesional arm. Therefore, the reaching distance was decreased to $10 \mathrm{~cm}$ only for the contralesional arm.

Participants were seated and instructed to reach to targets as quickly and as accurately as possible without moving their torso. We did not incorporate speed constraints on this task for any group, rather allowing them to move at their preferred speed. We believed that constraining young controls to reach slower to match stroke participants would change their normative motor control pattern and may cause larger inter-subject variability due to the different strategies that individuals may adopt to slow down their movements. The torso motion was restrained with Velcro straps attached to the vertical backrest of a chair. The participant's arm was visualized in VR as a "stick figure" connecting the positions of motion capture markers (Fig. 1B) and it moved concurrently with the participant at the temporal resolution of the VR helmet refresh rate ( 90 $\mathrm{Hz}$ ). Index fingertip was shown as a yellow ball $5 \mathrm{~cm}$ in diameter, and participants were instructed to move it into the center of each target. The intent of a large target size was to decrease the difficulty of the task, so that moderately impaired stroke participants would be able to perform it. Individual joint motion of the digits was not tracked; therefore, all participants were instructed to keep palm flat with all fingers extended and wrist pronated (palm down). The arm was not supported during reaching. Participants with stroke wore a finger splint made of polystyrene foam weighing < 10 gram to keep the digits 2-5 extended including at the metacarpophalangeal joints (palm down and open).

Each movement began with the participant's hand in the center target. A movement was cued by the center target changing color from green to red and the appearance of one peripheral green target (Fig. 1B). Upon detection of the index fingertip inside the peripheral target radius, its color changed to red, cueing the participant to return to the central target (Fig. $1 \mathrm{~A}$, end of center-out movement). After the participant reached the center target (Fig. $1 \mathrm{~A}$, end of return movement), the task reset, the peripheral target disappeared, and a new one appeared after a half-second delay. Movements to each peripheral target location were performed in a randomized order and repeated 15 times with rest breaks after bouts of 70 trials or upon 
request. Each participant repeated this experiment with both arms in separate sessions on different days.

\section{Data Collection and Processing}

All analyses and statistics were performed in MATLAB (Mathworks Inc.). Motion capture was recorded during the experiment using an active-marker motion capture system (Impulse by PhaseSpace). The light emitting diodes (active markers, LEDs) were placed according to best practice guidelines on anatomical bony landmarks of the arm and trunk (Robertson et al. 2013). Motion capture was collected at a rate of 480 frames per second, low pass filtered at $10 \mathrm{~Hz}$ and interpolated with a cubic spline (maximum interpolated gap: $0.2 \mathrm{~s}$ ). Data synchronization methods are described in detail in Talkington et_al. (4느). To improve the quality of kinematic data, spike artifacts detected at greater than 3 standard deviations at the elbow LED were removed automatically by blanking within .05 seconds of the spike before interpolation. Joint angles were calculated from motion capture using local coordinate systems defined as follows. Six LEDs on the clavicle, sternum, spine and the shoulder of the analyzed arm were used to define the trunk coordinate system. All joint angles were calculated relative to the trunk coordinate system, thus controlling for any change in trunk posture. The orientation of the trunk coordinate system was the same for both arms, therefore the directions of motion about the shoulder flexion/extension and internal/external rotation DOFs were opposite between limbs. They were flipped in the correlative analysis described below. Three LEDs, 2 on the shoulder and 1 on the elbow, were used to define upper arm coordinate system. Three LEDs, 1 on the elbow and 2 on the wrist, were used to define forearm coordinate system. Three LEDs, 2 on the wrist and 1 on the fingertip, were used to define hand coordinate system. The axes of the local coordinate systems were oriented in the same direction for both arms as shown in Fig. 1C. Joint angles were defined as Euler angles that corresponded to five joint degrees of freedom (DOFs) including 3 shoulder DOFs flexion/extension, abduction/adduction, internal/external rotation, 1 elbow DOF flexion/extension, and 1 wrist DOF flexion/extension. In some participants, the medial wrist LED was not reliably tracked due to being obscured frequently from camera view by moving body segments. Therefore, wrist pronation/supination DOF was not reliably detected and, thus, excluded from analysis. Wrist abduction/adduction was found to be minimal during these tasks and was likewise not included in the analysis.

The onset and offset of each center-out and return movements were identified from the differentiated trajectory of hand marker (velocity) crossing the threshold of $5 \%$ of maximal 
velocity at the beginning and the end of a given movement. Center-out movements toward one of the peripheral targets were separated from the return movements toward the central target by these events and analyzed as separate movements. The events were verified through visual inspection of the plotted trajectories to correct for unintended or corrective motion around the virtual target. The onset and offset events were used for temporal normalization of kinematic and torque profiles prior to averaging. Profiles starting $100 \mathrm{~ms}$ prior to the onset events were included in the analysis of each movement to capture the full profile of phase-advanced torques. All values included in text are means \pm standard deviation across participants, unless otherwise indicated.

\section{Inverse Dynamics}

The arm model was implemented in the Multibody toolbox of MATLAB. The model with 5 DOFs described in Olesh et al. (35) was used to calculate forces at the shoulder, elbow, and wrist joints. Joint angles for the shoulder, elbow, and wrist, obtained as described above were used to drive the model and simulate the center-out and return movements. The trunk was assumed to be stationary and in line with the world coordinate system (Fig. 1C). The inertia of major limb segments (humerus, radius/ulna, and hand) were modeled as ellipsoids with the long axes and masses scaled to the lengths of individual participants (Winter 2009). The segment diameters were kept constant at $10 \mathrm{~cm}, 6 \mathrm{~cm}$, and $6 \mathrm{~cm}$ for upper-arm, forearm, and hand, respectively. The short axes of the ellipsoids remained constant across participants. Inverse dynamics simulations using the individually scaled model shown in Fig. 1C were ran in Simulink. The output of these simulations was applied torques, not to be confused with net torques commonly referred to in Newton's laws, i.e., $t=I^{*} a$, where $t$ is net toque, $I$ is inertia, and $a$ is angular acceleration. The applied torques $\left(t_{a}\right)$ represent the active torques that need to be applied externally, in our case by muscles, to produce the desired motion of the limb in the presence of passive torques $\left(t_{p}\right)$ generated by the inertia and external forces, such as gravity. Torques summate, thus $\mathrm{t}=\mathrm{t}_{a}+\mathrm{t}_{p}$ or $\mathrm{t}_{a}=\mathrm{t}-\mathrm{t}_{p}$. We termed the applied torques muscle torques (MT). MT were further subdivided into two additive components, termed gravitational and dynamic components. The gravitational component (MTg) captured the portion of applied torque that supports the limb segments against the force of gravity. The dynamic component (MTd) captured the residual applied torque related to intersegmental coordination (Gottlieb et al. 1997; Russo et al. 2014). To obtain MTd, we ran the inverse dynamics simulations with the gravity of the physics engine set to zero (Olesh et al. 2017). As before, we took advantage of the additive nature of torques, deriving MTg by subtracting applied torques calculated in simulations without 
gravity from those calculated in simulations with gravity, i.e. $\mathrm{MTg}=\mathrm{MT}-\mathrm{MTd}$, for each DOF movement and subject.
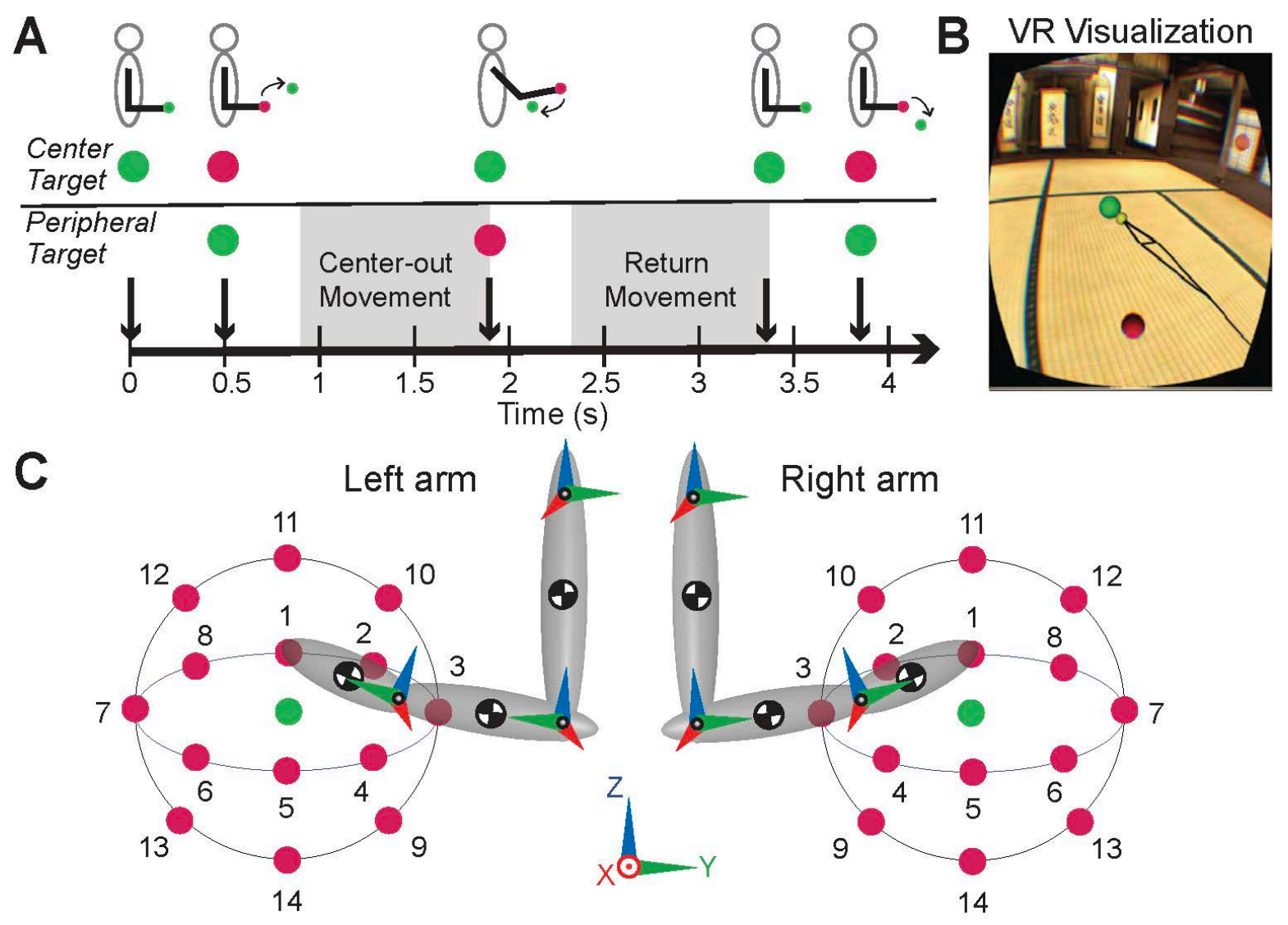

Figure 1. Standardized reaching in virtual reality. A. Trial timeline for each center-out and return reaching movement toward one of 14 virtual targets. B. Participant's VR view of the targets with visual feedback of their limb position (black "stick figure" indicating hand, forearm, and upper arm), the yellow sphere indicated the position of the tip of the index finger. $\boldsymbol{C}$. Side view of the scaled dynamic models of the right and left arms and relative target locations. The checkered circles represent the centers of mass of the segments. Arrows represent the orientations of local and world coordinate systems used for defining joint angles and the degrees of freedom for the right and left arms. Shoulder, elbow, and wrist flexion/extension degrees of freedom were calculated around $X(r e d)$ axes. Shoulder abduction/adduction degree of freedom was calculated around $Y$ (green) axis. Shoulder internal/external rotation degree of freedom was calculated around Z (blue) axis.

The quality of these simulations was checked by running forward simulations and calculating the root-mean-squared error (RMSE) between the endpoint trajectories obtained 
from motion capture and those from forward simulations. The RMSE mean \pm standard deviation across participants for the Control group was $1.4 \mathrm{e}-3 \pm 2.0 \mathrm{e}-3$ and $1.4 \mathrm{e}-4 \pm .5 \mathrm{e}-4 \mathrm{~mm}$ for the right and left arm respectively, for the Aged group it was 2.7e-4 $\pm 4.6 \mathrm{e}-4$ and $4.5 \mathrm{e}-05 \pm 4.7 \mathrm{e}-05$ $\mathrm{mm}$ for the right and left arm respectively, and for the Stroke group it was $4.9 \mathrm{e}-3 \pm 7.2 \mathrm{e}-3 \mathrm{~mm}$ and $0.3 \pm 0.8 \mathrm{~mm}$ for the ipsilesional and contralesional arm respectively. The RMSE values in the Stroke group were larger due to lower quality of motion capture data, where the markers disappeared from the view of cameras more often than during experiments with participants in other groups.

The movement time and endpoint accuracy were calculated for each participant. Endpoint accuracy was calculated as fingertip distance to the center of the outer targets at the end of the center-out reach phase, described above. Movement time was calculated as the elapsed time between the onset and offset events of each forward and return movement.

\section{Metrics}

Angular kinematic and torque profiles were normalized in time using onsets and offsets of each movement and resampled (1000 samples). Intertrial variability was calculated as the standard deviation of repetitions of individual movements toward the same target $(n=15)$ across the normalized trajectory, and then averaged over time. The inter-trial variability for wrist joint angle in young controls was $5.3 \pm 0.11$ and $5.7 \pm 0.16$ degrees for the right and left arm respectively. This was larger than what was observed in the DOFs of the shoulder (F/E R: $3.2 \pm$ 0.3, L: $3.4 \pm 0.36$; Ad/Ab R: $3.4 \pm 0.43$, L: $2.9 \pm 0.33$; InR/ExR R: $3.7 \pm 0.45$, L: $3.9 \pm 0.45$ degrees) and elbow ( $L: 3.8 \pm 0.5, R: 4.3 \pm 0.11$ degrees). Therefore, wrist DOF was removed from the rest of analysis. The individual normalized trajectories for shoulder and elbow were averaged to create a mean profile for each center-out and return movement toward each target for each participant. Muscle torque and muscle torque components were normalized by the subject-specific weight of each arm segment used during the inverse dynamic simulations, allowing for comparisons between subjects. From these, two types of metrics were derived, performance index and coefficient of determination

Performance index $(I)$ was calculated from the time-normalized trajectories of joint angles, angular velocities, muscle torques (MT) and their components (MTd and MTg) using the following formula adapted from (Fitts 1954) for each center-out or return movement to or from a given target per DOF of right or left arm for each subject:

$$
I=-\frac{1}{t} \log _{2} \frac{s}{2 A}
$$


where $t$ is the mean time to perform a given movement; $A$ is the peak-to-peak amplitude of a mean time-normalized trajectory for a given DOF, movement between central and a given outlying target (1 - 14 in Fig. 1), and subject, $s$ is the standard deviation of $A$ across repetitions of center-out or return movement. This means that for each subject's limb, 112 / values were calculated across 4 DOFs of shoulder and elbow and across 14 outward and 14 return movements. The units of the performance index are bits/s.

The coefficient of determination $\left(R^{2}\right)$ was calculated between the time-normalized trajectories of joint angles, angular velocities, muscle torques (MT) and their components (MTd and $\mathrm{MTg}$ ) for corresponding signals from the right and left limbs of the same participant. In this analysis, the participants served as their own controls, which reduced inter-subject variability. Mirror movements produced by left and right arms were matched as numbered in Fig. 1C because they were produced with the same joint angles and required contractions of the same muscles to perform them. $R^{2}$ ranges between 0 , indicating low symmetry between trajectories of right and left limb, and 1 indicating highly symmetrical trajectories. This means that for each subject, $112 \mathrm{R}^{2}$ values were calculated across 4 DOFs of shoulder and elbow and across 14 outward and 14 return movements.

\section{Statistical Analysis}

Data in Results are reported as means \pm standard deviation either across participants within each group or across movement directions for each individual, unless otherwise described. Paired t-tests were applied to all metrics comparing mean values across controls per movement direction and the corresponding data from the matching side in individuals in the Aged and/or Stroke groups. For these tests, all 112 I or $\mathrm{R}^{2}$ values were concatenated into a single array for each participant in the Aged and Stroke groups. To obtain control values, the values were averaged across participants in the Control group to generate 112 mean values for each DOF and movement direction. In Results, $p$ - values and $t$ - statistics are reported. Familywise error was corrected for multiple testing using Bonferroni method, i.e. a / N, where $\mathrm{N}$ is the number of subjects in either Aged or Stroke group. The significant a is reported in Results with every statistic.

To compare the sensitivity of distinguishing post-stroke motor deficits based on joint angles and MTd, we used k-means clustering to classify participants into one of 2 groups, those with motor deficits and those without motor deficits. Therefore, some aged and stroke participants could end up in the same cluster as young controls if their motor deficits are 
minimal. Clustering was applied to either angular or MTd $\mathrm{R}^{2}$ for each DOF of the shoulder and elbow per center-out and return movements per subject. To identify clusters, we used squared Euclidean distance measure with a heuristic approach for cluster centroid initialization (Arthur and Vassilvitskii 2007). The centroid coordinates in a 112-dimensional space were derived through an optimization process which minimized the distance from the centroid to each of the observations within the cluster. The reproducibility of cluster assignment was tested by running the algorithm 100 times and reporting the chances of aged individuals being classified into the same cluster as young controls and the chances of individuals with stroke being classified into a separate cluster from that with young controls.

Supplementary materials and original data are included in the GitHub repository https://github.com/NeuroRehabLab/Stroke Dynamic Scoring

\section{Results}

All participants in the Control group performed the center-out and return reaching movements with low inter-subject and inter-limb variability in endpoint trajectories (Fig. 2A). The reaches were performed with preferred speed, which differed somewhat between individuals. The endpoint accuracy defined as the distance from the center of the virtual target to the end of endpoint trajectory was $3.69 \pm 0.9 \mathrm{~cm}$ for right and $3.72 \pm 0.5 \mathrm{~cm}$ for left arm, well within the required tolerance. Joint angle trajectories mirrored the sigmoidal shape of the endpoint trajectories with classical bell-shaped velocity profiles but contained more detailed information about the motion of individual joints. Because movement amplitude, accuracy, and variability are inter-related, we quantified the performance of individuals using information theory as described in methods and earlier publications (Fitts 1954; Young et al. 2009). The performance index based on joint angles was $4.7 \pm 1.5$ bits/s (mean \pm standard deviation across individuals) for the right arm and $4.4 \pm 1.2$ bits/s for the left arm reaches. These values are much lower than values close to $10 \mathrm{bits} / \mathrm{s}$ reported for ballistic movements (Fitts 1954), suggesting that the reaching movements in our study had a low biomechanical complexity. A similar amount of information was reflected in the MTg trajectories (3.6 \pm 1.2 and $3.6 \pm 1.0 \mathrm{bits} / \mathrm{s}$ for right and left arm respectively), likely because this component is related to the cosine of the orientation of the segments to gravity, i.e., it is a dependent variable of joint angle. In contrast, higher information content was reflected in the trajectories of other variables. The performance index based on angular velocity was $5.8 \pm 1.6$ and $5.3 \pm 1.2$ bits/s for right and left arms respectively, and the performance index based MTd was $6.3 \pm 2.0$ and $5.7 \pm 1.4 \mathrm{bits} / \mathrm{s}$ for right and left arms respectively. As defined in Methods, muscle torque is the sum of MTg and MTd. Therefore, the 
MT performance index was intermediate between that derived from the two components at $4.0 \pm$ 1.6 and $4.1 \pm 1.3 \mathrm{bits} / \mathrm{s}$ for right and left arms respectively. The difference between the MTd and angular performance indices was $1.7 \pm 0.9$ for the right arm and $1.3 \pm 0.3$ for the left arm. This shows that the performance of reaching movements by the participants in the Control group can be characterized with higher information content using MTd trajectories compared to that using trajectories of joint angles.
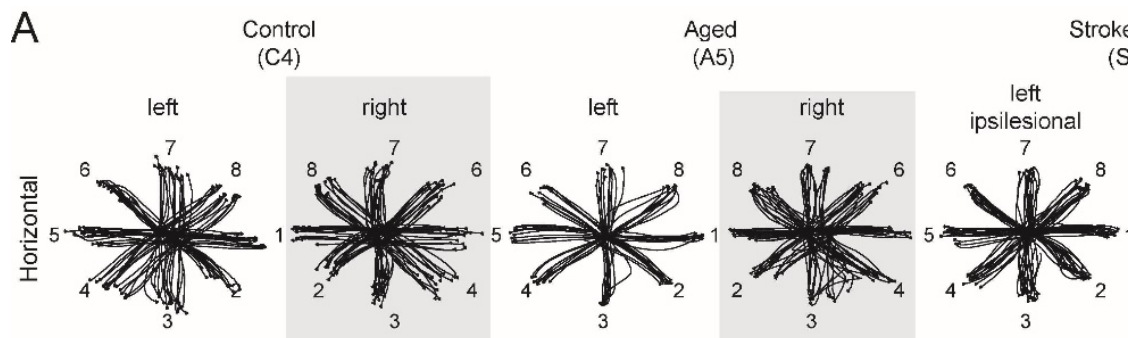

(S2)
(roke Mild
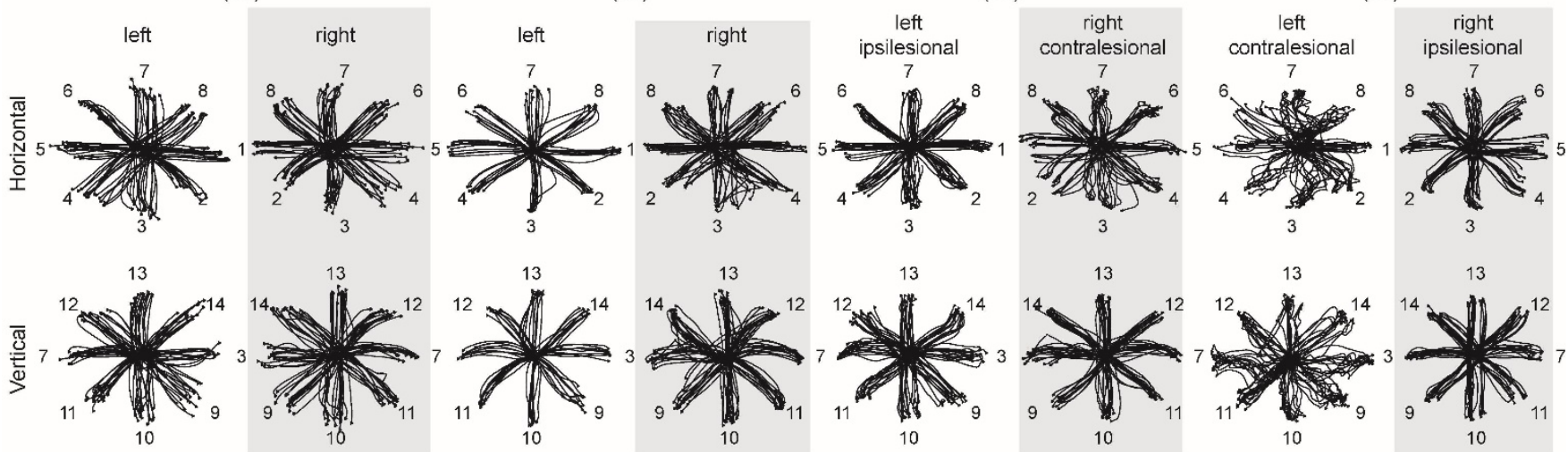

B
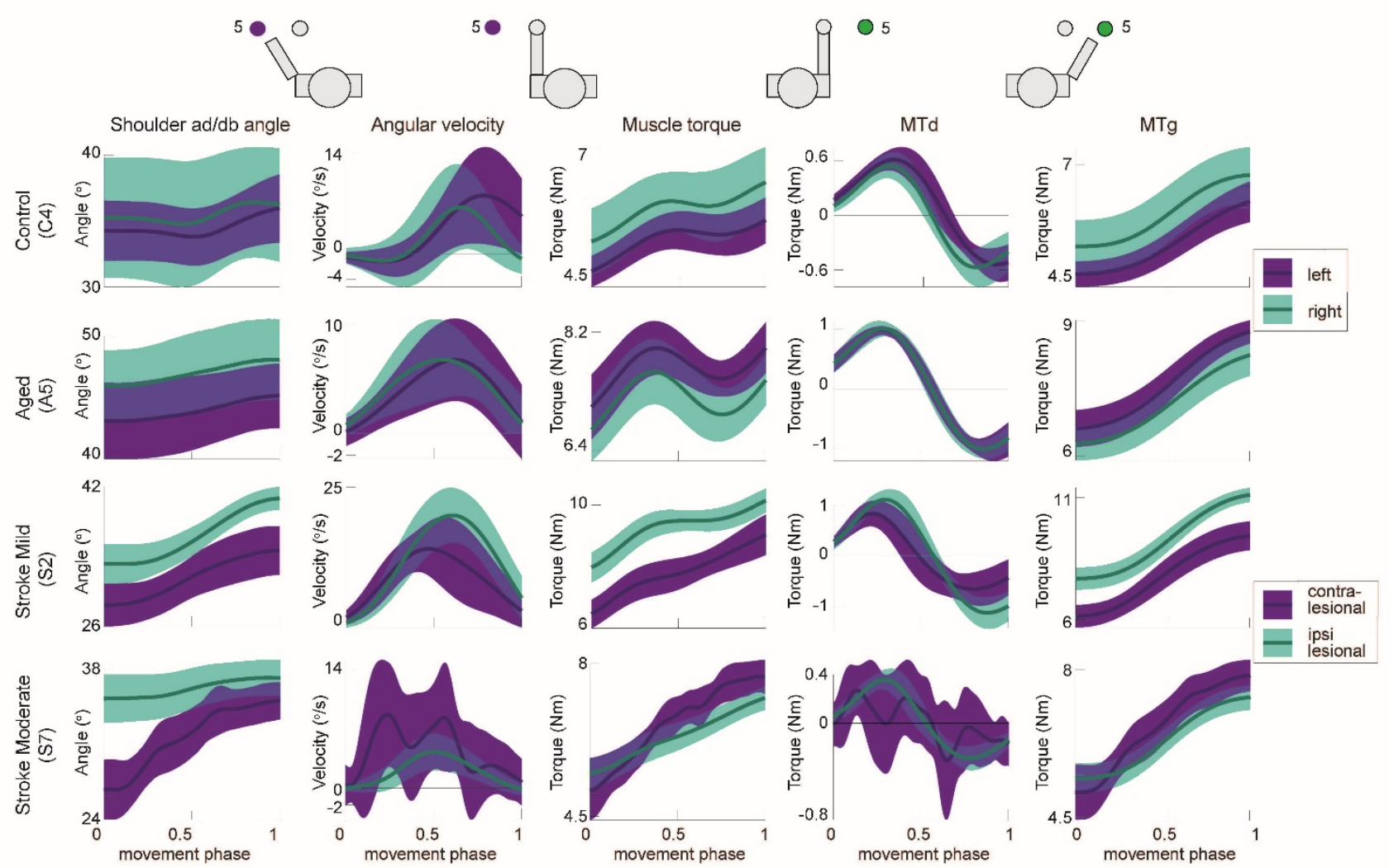
Figure 2. Kinematic and torque profiles of four participants. A. Endpoint trajectories for individual centerout reaching movement toward each of 14 peripheral targets. B. Example mean kinematic and dynamic trajectories from one movement, toward target 5 for left arm and toward target 7 for right arm. Mean trajectories (lines) and standard deviations (shaded areas) are shown for shoulder abduction/adduction DOF across 15 repetitions to each target.

The MTd trajectories are similar in shape to angular acceleration trajectories (Supplemental Fig. S1A). Therefore, the following analysis compares metrics derived from joint angle trajectories to not only metrics derived from MTd trajectories but also those derived from angular acceleration trajectories for corresponding joints. It is noteworthy, that the intertrial variability of acceleration trajectories was larger than that of MTd trajectories (Supplemental Fig. $\mathrm{S} 1 \mathrm{~B})$. This suggests that the metrics derived from angular acceleration may be less reliable than those derived from MTd.

As expected, all five participants in the Aged group were able to perform the center-out and return movements with matched reach amplitudes and target positions (Fig. 2A). The reaches were slower in 3 out of 5 aged participants compared to that for the corresponding arm of the Control group participants (A1 right arm $(\mathrm{R})$ : $t$-statistic from paired t-test $=4.1, p$-value $=$ 0.0004; A3 R: $t=9, p<0.0001$; A4 R: $t=13, p<0.0001$; and A1 left arm reach (L): $t=4, p=$ 0.0007 , significant $a=0.0042$ ). The endpoint accuracy of most aged participants was the same as in Controls, with the exception of $\mathrm{A} 2$, whose accuracy was worse than in controls $(\mathrm{R}: t=-6, p$ $<0.0001$ and $\mathrm{L}: t=-6, p<0.0038$ for all). This suggests that most participants in the Aged group reached slower, possibly to maintain the accuracy specified by the virtual targets, demonstrating the classical speed-accuracy tradeoff (Fitts 1954; Young et al. 2009). The angular performance index in aged participants was on average similar to young controls at 4.1 $\pm 1.3 \mathrm{bits} / \mathrm{s}$ for the right arm and $4.0 \pm 0.9 \mathrm{bits} / \mathrm{s}$ for the left arm reaches. As in the Control group, the MTd performance index was the largest among all indices at $5.1 \pm 1.6 \mathrm{bits} / \mathrm{s}$ for the right arm and $5.9 \pm 0.8 \mathrm{bits} / \mathrm{s}$ for the left arm reaches. The difference between the MTd and angular performance index was $1.0 \pm 0.4 \mathrm{bits} / \mathrm{s}$ for the right arm and $1.9 \pm 0.4 \mathrm{bits} / \mathrm{s}$ for the left arm.

Importantly, the right or left performance indices for reaching movements of all aged participants were different from those for movements with the corresponding arm of young controls, suggesting a propensity for asymmetric movement in the elderly. The angular performance index of all aged participants was different from that of Control group for reaching 
with one arm and sometimes both (A1 R: $t=10$, L: $t=6$; A2 L: $t=6$; A3 R: $t=6, \mathrm{~L}: t=-4 ; \mathrm{A} 4 \mathrm{R}$ : $t=7$ and A5 R: $t=-5$ ) with all $p$ were less than $\mathrm{a}=0.0042$ with Bonferroni correction for familywise error. The MTd performance index of aged participants was also higher that the angular performance index, just as was observed for the Control group. This increase was meaningful, as it showed larger changes in information content of the same aged participants (A1 R: $t=16, \mathrm{~L}: \mathrm{t}=6$; A2 R: $t=7, \mathrm{~L}: t=8$; A3 R: $t=15, \mathrm{~L}: t=-7$; A4 R: $t=15$ and A5 R: $t=-5$, $\mathrm{L}: t=-6, p<0.0042)$ as those identified by the angular performance index. This shows that the algorithm based on MTd trajectories is the most sensitive to individual differences in reaching.

All eight participants with stroke were able to perform the center-out and return movements with matched reach amplitudes and targets positions using their ipsilesional arm. The reaches were slower in 6 out of 8 stroke participants compared to that for the corresponding arm of controls (S1: $t=4$, S3: $t=13$, S5: $t=16$, S6: $t=6$, S7: $t=21$ and S8: $t=$ $12, p$ was less than the significant $a=0.0031$ for all). The endpoint accuracy of reaching with the ipsilesional arm was similar to the accuracy of reaching with the corresponding arm in Controls possibly due to the speed-accuracy tradeoff (Fitts 1954; Young et al. 2009). The angular performance index for the ipsilesional arm was on average lower than that for the corresponding arm of aged and young controls at $3.3 \pm 1.2 \mathrm{bits} / \mathrm{s}$. As in the controls, the MTd performance index was the largest among all indices at $4.1 \pm 1.4 \mathrm{bits} / \mathrm{s}$ for the ipsilesional arm with a difference between them at $0.9 \pm 0.4 \mathrm{bits} / \mathrm{s}$. The angular performance index for the ipsilesional arm was significantly different form aged controls in 4 participants with stroke (S4 $t=$ -5 , S5 $t=4$, S7 $t=11$, and S8 $t=8, p<0.0031$ for all). These differences in S7 and S8 were larger when compared to the corresponding mean performance index of the Control group (data not shown). The differences in MTd performance index for the ipsilateral arm were significant for 5 participants with stroke (S3 $t=5, \mathrm{~S} 4 t=-5, \mathrm{~S} 5 t=8, \mathrm{~S} 7 t=19$ and S8 $t=13, p<0.0031$ for all), only some of them moved slower. The same was true for the comparison with the Control group (data not shown). Once again, the sensitivity of the algorithm based on MTd trajectories was most sensitive to the individual differences in reaching compared to that based on trajectories of angles. Overall, this shows that the changes in reaching with the ipsilesional arm in 5 (S3, S4, S5, S7 \& S8) out of 8 participants with stroke were different from the changes in reaching associated with age and different from reaching by young controls. In the rest of participants with stroke (S1, S2 and S6), reaching with the ipsilesional arm was similar to that of aged or young controls. 
All but two participants in the Stroke group were able to perform the center-out and return movements with matched reach amplitudes and targets positions using their contralesional arm (Fig. 2A; Supplemental Fig. S2). The movement speed of reaching with the contralesional 'impaired' arm was lower than that of the Control group (Table 1). The contralesional reach durations were longer in all but three participants with stroke compared to that for the matching arm in Control group (Table 1; S1 $t=7$, S2 $t=9$, S3 $t=10$, S5 $t=10$, and S7 $t=17, p<0.0031$ for all). However, the endpoint accuracy of reaching with contralesional arm was similar to that of the Control group in all participants with stroke (Table 1) possibly due to the speed-accuracy tradeoff. Of note is that the timing and accuracy measures are confounded by the shorter reach distances of one participant (S8). The performance index controls for these confounding factors. The angular performance index for the contralesional arm was on average lower than that for the corresponding arm of aged and young controls at $3.2 \pm 1.2 \mathrm{bits} / \mathrm{s}$. As in the controls, the MTd performance index was the largest among all indices at $4.7 \pm 1.7 \mathrm{bits} / \mathrm{s}$ for the contralesional arm with a difference between them at $1.5 \pm 1.4 \mathrm{bits} / \mathrm{s}$. Angular performance index for the contralesional arm was lower in all but 2 participants with stroke compared to that for the corresponding arm of participants in the Aged group (S2 $t=6$, S3 $t=6$, S $4 t=-4$, S5 $t=10$, S $7 t=12$ and S $t=4, p<0.0031$ for all). Comparison to the Control group revealed similar differences (data not shown). Moreover, MTd performance index revealed larger positive differences in $\mathrm{S} 1$ and in most participants with significant differences in their angular performance index (S1 $t=6, \mathrm{~S} 2 t=24, \mathrm{~S} 3 t=18, \mathrm{~S} 4 t=-3, \mathrm{~S} 5 t=34$ and S7 $t=$ $41, p<0.0031$ for all) with the exception of $S 8$. Comparison with the Control group revealed similar differences (Fig. 3). In conclusion, altogether these metrics have shown that reaching with the contralesional arm in most participants was different from that in aged and young controls with the exception of S6. 


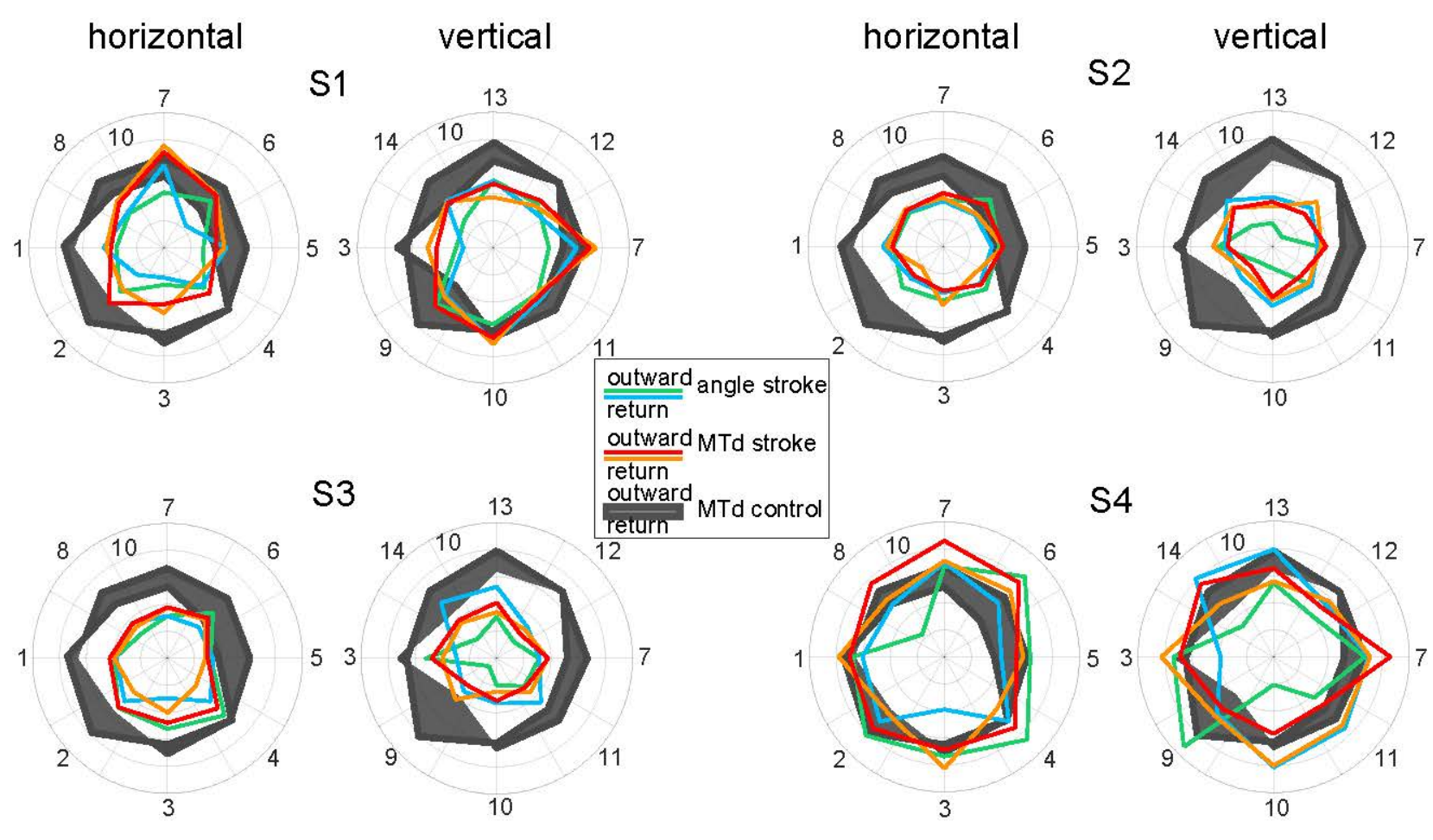

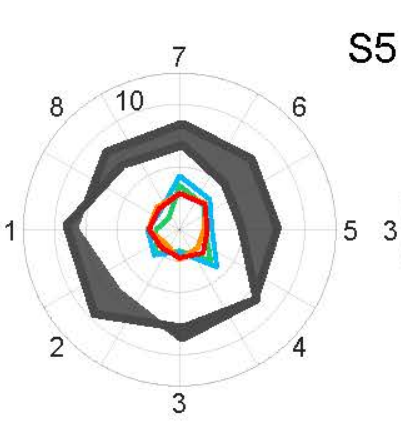

S5

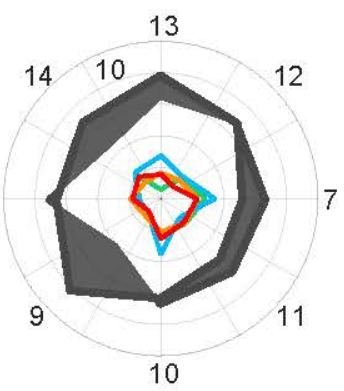

S7
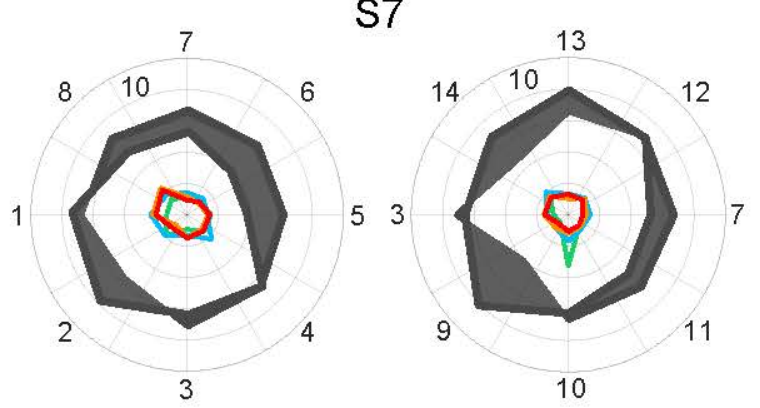

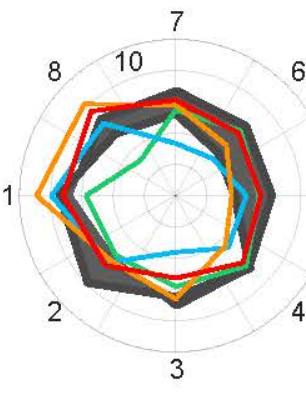

S6

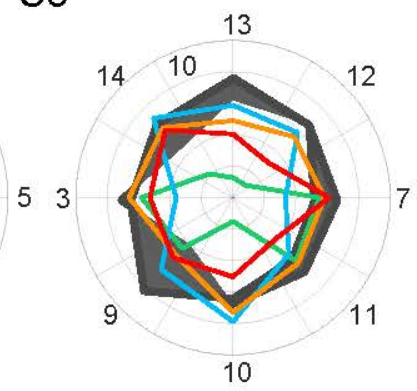

S8

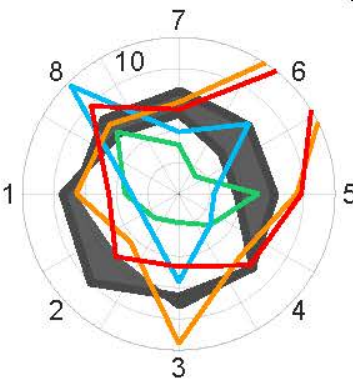

Figure 3. Performance index for each movement direction per participant with stroke. Each plot shows angular performance index (blue and green lines for outward and return movements respectively) and MTd performance index (red and orange lines for outward and return movements respectively) for reaching with the contralesional arm. Black lines with shaded area denote the mean MTd performance 
indices for outward and return reaches with the left hand by young controls. The outer radius in all plots is equal to $10 \mathrm{bits} / \mathrm{s}$.

The MTd performance index was similar across movement directions for both outward and return movements in young controls (Fig. 3, grey shading). In contrast, the angular performance index was not uniformly distributed across movement directions, being close to zero for center-out movements toward targets 4 (toward the body and to the right), 9 (toward the body and down), and 10 (toward the body and up, data not shown). However, in the participants with stroke the performance index for their contralesional arm was uniformly lower than in controls for some (S2, S3, S5 and S7) and non-uniformly distributed across movements for others (S1, S4, S6 and S8; Fig. 3). This illustrates the heterogeneity of individual stroke pathologies. The amplitudes of the angular and MTd performance indices also varied independently across different movement directions, indicating that the changes in the angular excursions for movement in different directions are not always accompanied by pronounced changes in the amplitude of joint torques. The converse is also true, so that in some movements during which some joint angles do not change do required different amplitudes of joint torques. Thus, angular and torque trajectories contain fundamentally different information about deficits when moving in different directions. The acceleration-based performance index was generally lower than the MTd performance index in stroke participants (Supplemental Fig. S3). This is likely due to the higher intertrial variability of angular acceleration profiles compared to that of MTd profiles. However, the acceleration-based performance index contains similar information about deficits when moving in different directions.

According to Eq. (1), the reduction in the performance index can be driven by the increased time to make the movements, increased trajectory amplitude, and decreased intertrial variability. The latter can be excluded from reducing the performance index as inter-trial variability was increased rather than decreased in participants with stroke (data not shown). Also, the amplitudes of joint angle trajectories were constrained to be the same by our VR task across all participants except S8, who did not show uniform reduction of his performance indices (Fig. 3, bottom right corner). This constraint also helps to exclude the amplitude of movement from causing the reduction of the performance index. Therefore, for S3, S5, and S7 the decrease in the performance index for the contralesional arm was driven by slower reaching movements. For the rest of the participants with stroke (S1, S2, S4, and S8), the changes in the 
performance index were driven by the changes in the ratio between inter-trial variability and the trajectory amplitude. Note, that $\mathbf{S 6}$ showed no changes in performance index for either arm.
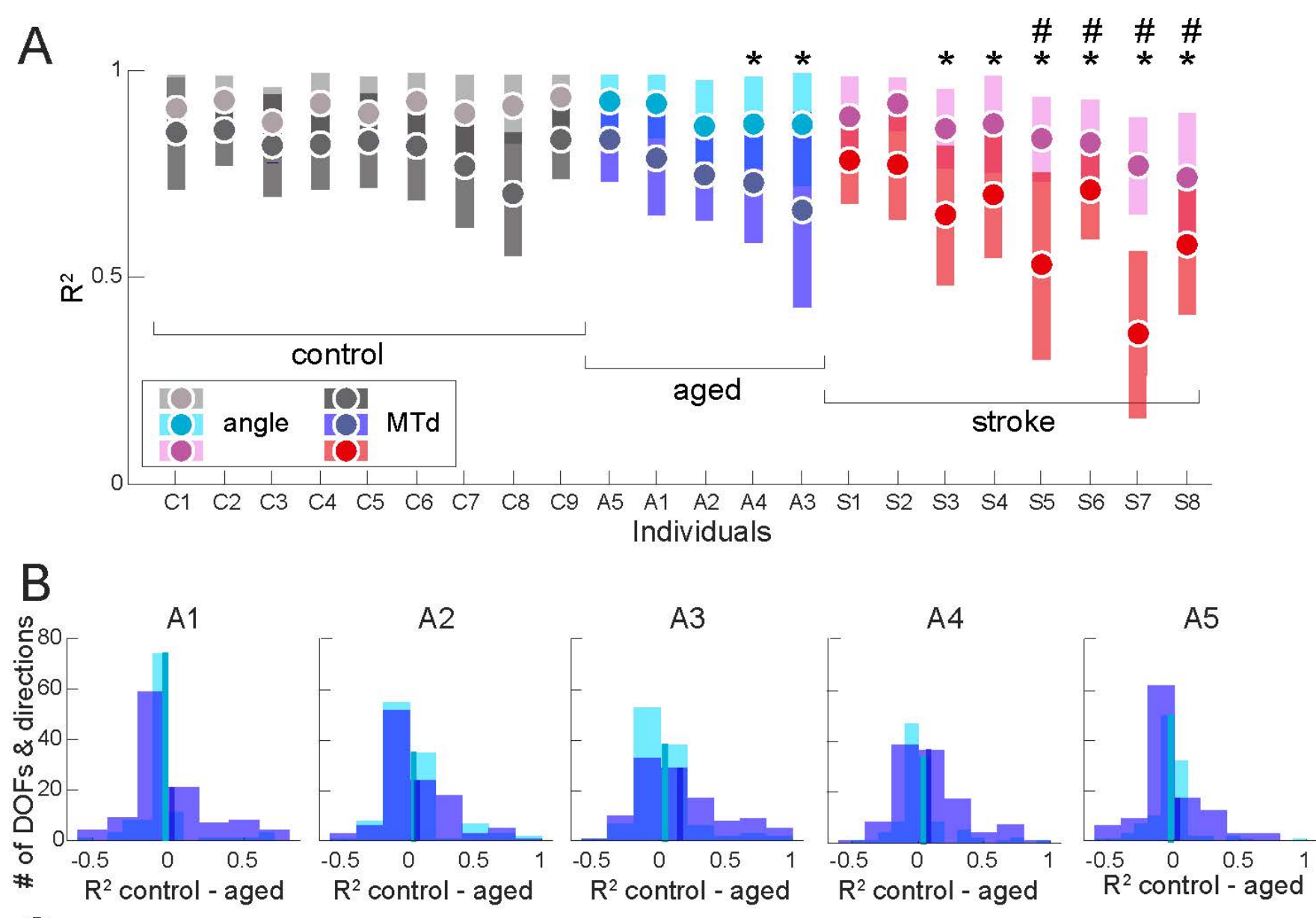

C
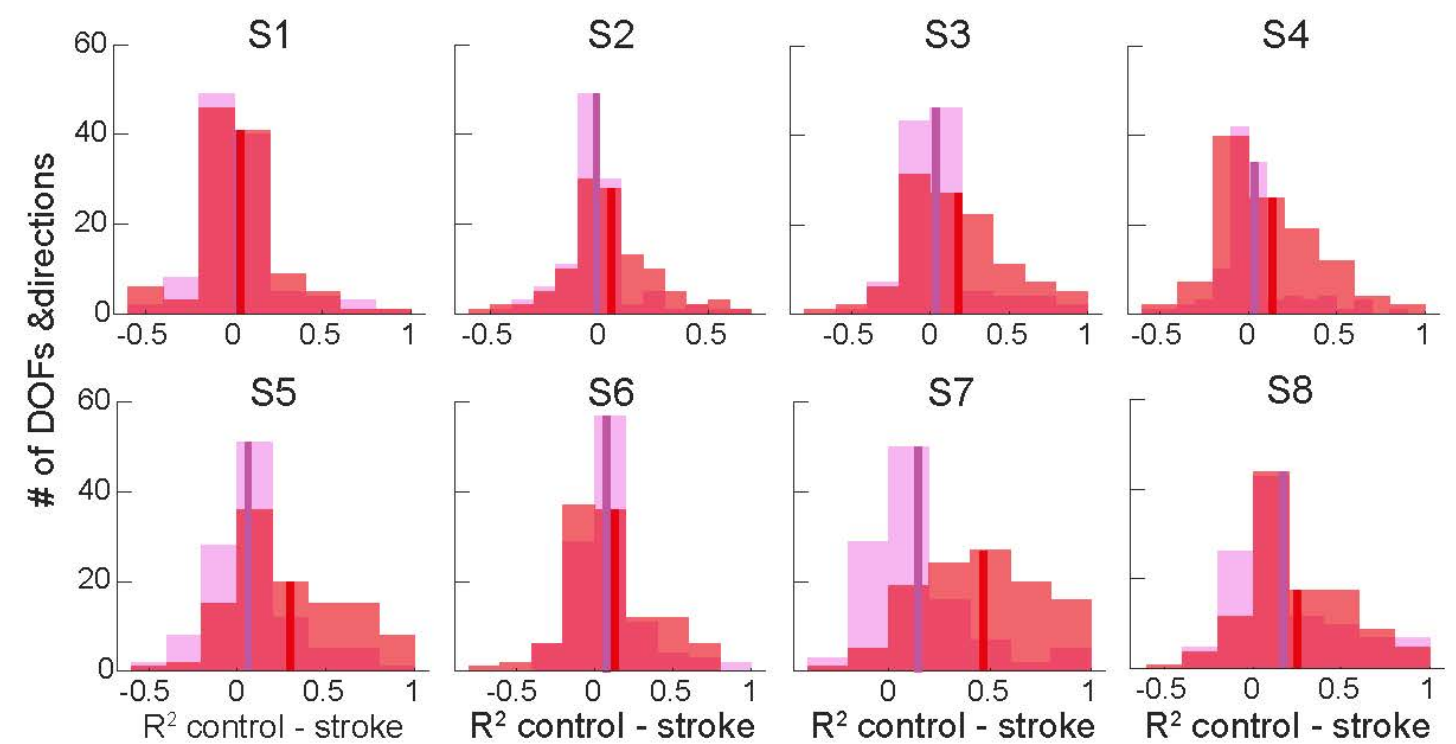
Figure 4. Coefficient of determination $\left(R^{2}\right)$. A. Individual $R^{2}$ based on joint angle and MTd. The central tendencies of data per participant are shown as means (dark circles) with standard deviation across movement directions (light bars). DOFs were averaged to show variation across each movement direction. Paired T-tests were used to compare individuals to controls, without averaging across DOF (28 movement directions for $4 D O F, n=112)$. Hashtags show significant differences between angle $R^{2}$ in individuals with stroke compared to angle $R^{2}$ averaged across young controls per movement direction and DOF. Stars show significant differences between $M T d R^{2}$ in aged individuals and those with stroke compared to $M T d R^{2}$ averaged across young controls per movement direction and DOF. Significant $a=$ 0.0031 with correction for multiple tests. $B$. Differences between $R^{2}$ in aged and young controls per movement direction per DOF. Colors are as in A. C. Differences between $R^{2}$ in individuals with stroke and young controls per movement direction per DOF. Colors are as in A, with vertical lines indicating means.

It is difficult to distinguish the mild motor deficits caused by stroke or aging from the normal variability in how individuals make movements. Therefore, we next quantified how symmetrical the reaching trajectories were between the right and left limb using the coefficient of determination $\left(R^{2}\right)$ that controls for inter-subject variability. The profiles of joint angles were highly symmetrical between limbs in young and some aged controls and less symmetrical in most participants with stroke (Fig. 4A). The angle $R^{2}$ values tended to saturate at 1 , likely due to the lower information content of angular trajectories compared to the MTd trajectories. In contrast, the profiles of MTd were less symmetrical in young controls so that the coefficient of determination was more variable between individuals and less likely to saturate (Fig. 4A). This asymmetricity in MTd profiles between right and left arms in young controls is likely due to dominance effects. The MTd $\mathrm{R}^{2}$ values were similar to those based on angular acceleration for most participants (Supplemental Fig. S4). Moreover, the differences in MTd $\mathrm{R}^{2}$ values between aged and young participants were larger in more movements than the corresponding differences in angle $R^{2}$ (Fig. 4B). Specifically, for aged participants A3 and A4 the profiles of MTd were significantly less symmetrical than in young controls (paired t-tests between mean $\mathrm{R}^{2}$ per movement direction in young controls and A3 $t=5$ and A4 $t=3, p<$ significant a of 0.0031 for both). This supports the propensity for asymmetric movement in these aged participants identified with the unilateral changes in performance index. However, the bilateral changes in performance index in aged participants $\mathrm{A} 1, \mathrm{~A} 2$, and $\mathrm{A} 5$ were not accompanied by the asymmetric MTd profiles, indicating that in these aged participants the bilateral age-related 
changes in reaching movements were similar in both limbs. Overall, similar to the conclusions from performance analysis, the differences between young controls and aged individuals were greatly amplified when quantified with the coefficient of determination based on MTd or angular acceleration.

For most participants with stroke, the profiles of both angle and MTd were less symmetrical than the mean $\mathrm{R}^{2}$ in young controls (Fig. 4B). For four participants with stroke the angle $\mathrm{R}^{2}$ was less symmetrical than the corresponding values in young controls (S5 $t=3$, S6 $t=$ 4, S7 $t=6$, and S8 $t=6, p<$ significant a of 0.0031 for all). Furthermore, for six participants with stroke the MTd $R^{2}$ was less symmetrical than the corresponding mean $R^{2}$ in young controls ( 33 $t=5$, S4 $t=4$, S5 $t=10$, S6 $t=4, \mathrm{~S} 7 t=17$, and S8 $t=9, p<$ significant a of 0.0031 for all). This shows again that the differences between young controls and most individuals with stroke were amplified when quantified with the coefficient of determination based on MTd with the exception of S6. Note, that the performance index of S6 was not different from aged nor young controls, therefore the interlimb asymmetricity of his reaching likely indicates mild hemiparesis. Interestingly, the performance index for S1 and S2 indicated significant differences in reaching with the contralesional arm but not ipsilesional arm. However, the $\mathrm{R}^{2}$ values for these participants were not significantly different from young controls, indicating that the shapes of angular and MTd trajectory waveforms were on average symmetrical between limbs. The differences in performance index in these participants were driven by higher inter-trial variability. However, the high symmetricity of their reaching trajectories indicates that they do not have pronounced deficits in intersegmental coordination. In contrast, the rest of the participants had both significant performance index changes for their contralesional arm driven by both slower movements and increased variability, and they also had lower interlimb symmetry in their MTd profiles, indicating larger deficits in intersegmental coordination of the contralesional arm. 

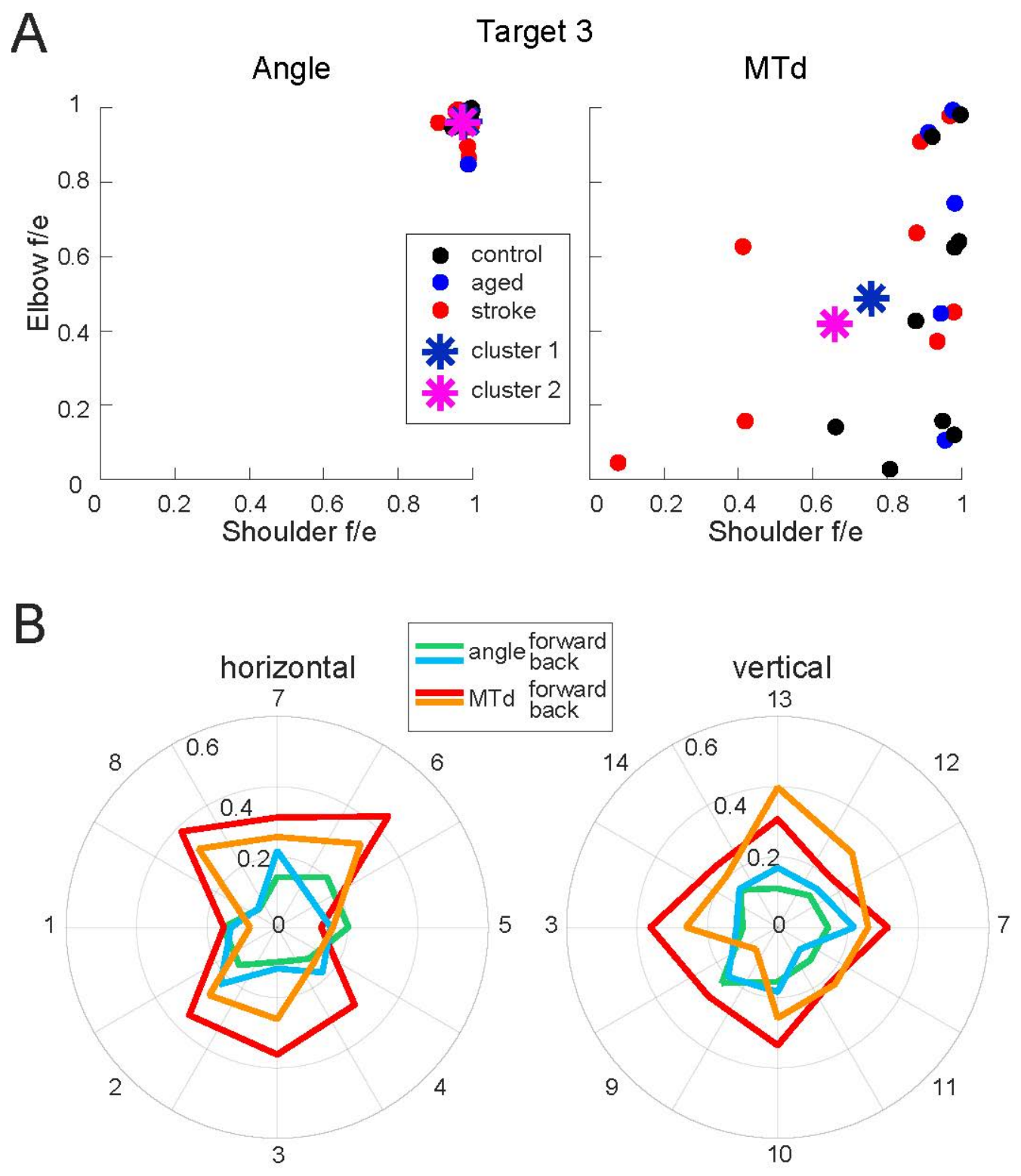

Figure 5. Cluster analysis of the coefficient of determination. A. A projection of cluster centroid locations (stars) and individual $R^{2}$ values (filled circles) onto only 2 dimensions (shoulder and elbow flexion/extension DOFs) is plotted for a single movement toward target 3 . The colors of circles indicate participant group allocation, not cluster allocation. This plot is a partial view of the clustering analysis reported in Results. Left plot shows results based on angle $R^{2}$ values, while right plot shows results based on MTd $R^{2}$ values B. Distances between the two clusters per movement direction averaged across DOFs. This is a more high-level overview of the clustering analysis than $\boldsymbol{A}$. Blue and green lines show distances between clusters of angular $R^{2}$ values for center-out and return movements respectively. Red and orange lines show distances between clusters of $M T d R^{2}$ values for center-out and return movements respectively. 
To further distinguish between motor deficits caused by stroke from changes in movements due to inter-subject variability, the $M T d$ and angle $R^{2}$ values were used to classify individual participants into two groups. K-means clustering analysis relies on an optimization algorithm, which can converge on different solutions. Therefore, the clustering was repeated 100 times. The distance between the two clusters into which all participants were grouped was larger when the analysis was based on $\mathrm{MTd} \mathrm{R}^{2}$ at $2.94 \pm 0.64$ across multiple optimizations compared to the analysis based on angle $\mathrm{R}^{2}$ at $1.61 \pm 0.38$ (one-tailed t-test $t=16, p=2 \cdot 10^{-37}$ ). The distances between the two clusters based on MTd $\mathrm{R}^{2}$ were consistently larger than those based on angle $\mathrm{R}^{2}$ across most movement directions (Fig. 5). The small distance between clusters based on angle $\mathrm{R}^{2}$ values is likely because of these values saturating. Consequently, young controls were grouped together $53-63 \%$ when clustering was done on angle $\mathrm{R}^{2}$ in contrast to $90-98 \%$ when clustering was done on $M T d R^{2}$. Similar increases in classification performance were evident for aged participants being grouped together with young controls (angle $\mathrm{R}^{2}: 43$ - $64 \%$, MTd $\mathrm{R}^{2}: 66$ - $95 \%$ ). Differences in performance indices in participants A2, A3 and A4 and in interlimb asymmetricities in A3 and A4 resulted in these aged participants being grouped less often with young controls based on $\mathrm{MTd}^{2}(\mathrm{~A} 2: 82 \%, \mathrm{~A} 3: 66 \%$ and $\mathrm{A} 4$ : $86 \%$ compared to $\mathrm{A} 1: 93 \%$ and $\mathrm{A} 5: 95 \%)$. This was not as apparent from clustering based on angle $R^{2}(A 2: 55 \%, A 3: 57 \%$ and $A 4: 43 \%$ compared to $A 1: 64 \%$ and $A 5: 56 \%)$. The classification based on MTd $\mathrm{R}^{2}$ was also robust for individuals with stroke. The participants with stroke whose motor deficits were minor were rarely separated from controls (S1: $4 \%, \mathrm{~S} 2: 1 \%$, and S6: 17\%), while the participants with stroke whose motor deficits were larger were separated from controls more often (S3: $42 \%$, S4: $27 \%$, S5: $86 \%$, S7: $82 \%$, and S8: $66 \%$ ). Clustering of acceleration $R^{2}$ values gave largely similar results as that for $M T d R^{2}$ values (Supplemental Fig. 5). However, the optimization algorithm converged on more variable range of solutions when clustering acceleration $R^{2}$ values compared to that for $M T d R^{2}$ values. This evidence further supports the conclusion that algorithms based on MTd are the most sensitive to subtle differences in reaching movements and able to separate pathological motor deficits from typical differences in how movements are produced by individuals. 


\section{Discussion}

Here we have objectively quantified the individual differences in reaching that could underlie motor deficits caused by aging and stroke. We have shown that joint torques comprise a more sensitive measure of individual differences in reaching compared to joint angles. This confirms our main hypothesis that muscle torques contain more information about post-stroke motor deficits than angular kinematics. The profiles of the dynamic component of muscle torque contain more information than joint angles, as evidenced by the larger performance index based on the former compared to the latter in all participants. Furthermore, the profiles of the dynamic component of muscle torque were the most sensitive to the individual differences in reaching symmetricity, as evidenced by the lowest coefficient of determination values. Furthermore, the clustering analysis has shown that the coefficient of determination based on the dynamic component of muscle torque could be used to distinguish the individual differences in reaching due to aging or stroke from those caused by typical inter-subject variability. Lastly, we have shown that the metrics derived from angular acceleration contain similar information to the metrics derived from the dynamic component of muscle torque, but the former capture subtle differences in reaching between individuals with less sensitivity than the latter.

The assessment of motor deficits using torques or angular accelerations may be especially useful for patients who have less observable deficits, such as those classified as asymptomatic via traditional motion-based assessments, but who may still report difficulty moving, increased fatigue, and/or inactivity. Low-cost commercial motion capture devices in combination with powerful computing devices that are capable of running sophisticated algorithms are becoming widely available for home use, technology driven rapidly by the gaming industry. Our study has shown that estimating angular acceleration or muscle forces that drive motion can enable a new type of automated assessment of age-related changes in movements and post-stroke motor deficits. This approach can be potentially useful as part of telemedicine and mobile health initiatives, where the patient's health needs to be monitored remotely.

Interestingly, although the temporal profiles of the dynamic component of muscle torque are similar to the profiles of angular acceleration for corresponding DOFs, the latter has higher intertrial variability than the former. This suggests that the active torques resulting from muscle contractions are less variable than the net torques, which are proportional to angular acceleration as stated in the Newton's 2nd Law of Motion. Therefore, our analysis has shown that although the temporal profiles of joint angular acceleration do contain a similar amount of information as the dynamic component of muscle torque, the increased variability reduces the 
sensitivity of metrics derived from acceleration compared to those derived from the dynamic component of muscle torque. However, this limitation may be compensated for the simplified analysis based on angular acceleration that does not require a dynamical model or a physics engine. Overall, both an acceleration-based and a force-based assessment developed based on our metrics could improve the sensitivity of the objective measurement of individual motor impairment.

Most aged participants reached more slowly than young controls despite instructions of reaching as fast as possible. This may be due to an attempt to maintain the accuracy specified by the virtual targets, possibly due to the classical speed-accuracy tradeoff (Fitts 1954; Young et al. 2009). Alternatively, the slower movement of the elderly may be due to the addition of multiple corrective sub-movements within each reach (Ketcham et al. 2002). In all but one aged participant there were lower performance indices in at least one and sometimes both arms. In the one aged participant (A5 59-year-old male) the performance index was higher than the mean of young controls in corresponding movements for both arms, and the movements were as symmetrical as those of young controls, which suggests that this participant did not have motor deficits but rather performed the movements using a different strategy. Two other aged participants ( $\mathrm{A} 3$ and $\mathrm{A} 4$, both 58-year-old females) performed reaching movements less symmetrically than young controls with lower performance indices for their dominant right arms. The rest of the aged participants (male A1 and female A2, both 58-year-old) had symmetrical deficits so that their performance indices were lower in both arms. Studies with planar goaldirected movements that control for the effect of gravity have indicated that the neural compensation for intersegmental dynamics for the non-dominant limb is worse than that for the dominant limb_(Bagesteiro and Sainburg 2002; Sainburg 2002; Sainburg and Kalakanis 2000). Our results show differences between limbs in young and aged control participants that are most pronounced in MTd $\mathrm{R}^{2}$. This supports the published observations because the dynamic component of muscle torque reflects active compensation for intersegmental dynamics (Debicki and Gribble 2005; Gribble and Ostry 1999; Ketcham et al. 2004; Pigeon et al. 2003). However, these differences in our small sample of aged participants were not specific to one limb. In A1 the bilateral reduction in performance index was due to the reduced speed of movement, while in A2 this was due to the increased variance of movement. The clustering analysis grouped participants $A 1$ and $A 5$ primarily with young controls, indicating that their sometimes slower (A1) and quirky (A5) movements were part of typical differences in how movements are produced by individuals rather than an indication of age-related motor deficits. The differences between aged and young participants were larger when measured from MTd compared to joint angle, 
indicating that the force-based measures are more sensitive to age-related motor deficits than motion-based measures are. Altogether, this shows that force-based metrics can be used to characterize with high sensitivity the individual differences in reaching movements associated with aging and distinguish them from typical inter-subject variability. However, more research is needed to establish the relationship between the age-related motor deficits and hemispheric dominance.

Here we show that force-based measurements of post-stroke motor deficits are more sensitive to individual pathology compared to motion-based measurements. In four participants with stroke (S3 and S7 with a stroke in medulla and S5 and S8 with a stroke in left and right MCA respectively, Table 1) the hemiparesis affected movements of both limbs as shown by reduced performance index for both limbs and reduced symmetricity of movements between limbs. In S4 with a lacunar infarct in posterior right putamen, the performance index of the ipsilesional arm was higher than that for aged participants and similar to that for young controls, indicating a different strategy for reaching movements, possibly similar to $A 5$, rather than the presence of motor deficits in the ipsilesional arm. In S6 with a right MCA stroke the performance index for the ipsilesional arm was not different from that for aged nor for young participants, also indicating the absence of detectable motor deficits in the ipsilesional arm. However, the MTd $\mathrm{R}^{2}$ in both these participants was lower than in young controls, indicating that their movements were asymmetrical. In the last two participants with stroke (S1 with a left MCA stroke and S2 with a left caudate lenticular nucleus stroke), the effect of stroke on their reaching with either arm was very mild. Their reaching was slower, which reduced the performance indices for their contralesional arms. However, their movements were symmetrical with $M T d R^{2}$ indistinguishable from young participants. The clustering analysis grouped participants $S 1, \mathbf{S 2}$, and S6 primarily with young controls, indicating that their sometimes slower (S1 and S2) and asymmetrical (S6) movements were part of typical differences in how reaching movements are produced by older individuals rather than an indication of motor deficits caused by stroke. The differences between participants with stroke and young controls were larger when measured from MTd compared to joint angle. This indicates that the force-based measures of individual motor deficits due to stroke are much more sensitive than motion-based measures in quantifying the subtle multi-joint differences in complex 3D reaching movements. This also suggests a method for distinguishing the individual motor deficits caused by stroke from the typical differences in how movements are produced by individuals and from age-related changes in movement production. 
A limitation of this study is that the movement differences between individuals captured here are not easily generalizable to the levels of impairment or functional ability typically quantified with clinical scales. A prior study has quantified a linear relationship between movement differences measured with the coefficient of determination used in our study and upper limb impairment quantified with the Fugl-Meyer Test (Olesh et al. 2014). Using this relationship from Fig. 3B of Olesh et al. (Olesh et al. 2014), we can estimate that the average score for the upper-limb tasks of FMA for our participants ranged from 2.4 to 3.0, putting all our participants with stroke in the low to moderate impairment category. Therefore, more research is needed to generalize the results of our study to the people with moderate to high impairment levels caused by chronic hemiparesis. A related limitation is that only a simple reaching task was included in our study. More variety of functional motor tasks are needed that include real object interactions to test the generalization of our conclusions.

The dynamic component of active muscle torque is indicative of intersegmental coordination, something that cannot be easily inferred from movement observation. Our results have shown that most Stroke group participants show a deficit in all metrics derived from MTd. This is consistent with published observations showing deficits in intersegmental coordination and the phasic muscle activity patterns after a stroke during the performance of planar reaching tasks and some unconstrained reaching tasks (Bastian et al. 1996; Beer et al. 2000; Cirstea et al. 2003; Levin 1996; Manto and Bosse 2003). Furthermore, the deficits measured with MTd were generally present for all movement directions (Fig. 3, 4C, and 5B), indicating that the disruption in intersegmental coordination caused by stroke is general and can be observed in most reaching movements regardless of the starting position, speed, and direction of reaching. This shows for the first time that the deficits in intersegmental coordination caused by stroke can be quantified in unconstrained 3D reaching movements while controlling for posture-dependent forces and other sources of inter-subject variability. This method for quantifying intersegmental coordination may help increase the efficacy of robotic or robot-assisted post-stroke rehabilitation (Patton et al. 2006; Sanchez et al. 2006; Volpe et al. 1999, 2000, 2001). New approaches for designing the pattern of robot assistance or resistance to appropriately titrate the difficulty of therapy while avoiding moving the arm passively, are needed (Kahn et al. 2014; Lum et al. 2002). Quantifying deficits in the dynamic component of active muscle torque and setting robotic devices to assist or resist only this component may be a way to personalize and standardize intervention and improve the recovery of intersegmental coordination. 
Furthermore, quantifying the dynamic component of muscle torque while controlling for the gravity-compensating component enables direct comparison between unassisted and unconstrained 3D movement and movements performed with support against gravity or other external assistance or resistance. In particular, supported planar 2D reaching tasks are now used more widely for robot assisted therapy in combination with quantitative metrics derived from the same planar tasks (Coderre et al. 2010; Kwakkel et al. 2019; Scott and Norman 2003). However, these movements are constrained and not overtly functional, which makes it difficult to quantify the carry over effect from therapy based on planar movements into real-world effects on 3D functional movements in a meaningful way that helps inform future interventions. The method used here to derive quantitative assessment metrics from the individual dynamic component of muscle torque can help bridge the gap between evidence from studies of constrained or robotically manipulated movements and research with functional and unconstrained movements.

\section{Acknowledgments}

We thank the study participants for generously giving their time, and M. Powers for her assistance with participant recruitment and the general support of the study; we acknowledge the technical support expertly provided by B. Pollard; we are thankful for insightful and critical comments of S. Yakovenko.

\section{Funding}

VG was supported by grants P20GM109098 and P30GM103503 from the National Institute of General Medical Sciences (https://www.nigms.nih.gov). ABT was supported by the training grant T32AG052375 from the National Institute of General Medical Sciences. EVO was supported by a training grant T32GM081741 from the National Institute of General Medical Sciences. AA was supported by grant U54GM104942 from the National Institute of General Medical Sciences. The funders had no role in study design, data collection and analysis, decision to publish, or preparation of the manuscript.

\section{Disclosures}

None 


\section{Chapter 3: Designing an Assistive Device for stroke rehabilitation.}

Title: Gravity support at the shoulder using Functional Electrical Stimulation during reaching facilitates improved muscle coordination after stroke.

Authors: Ariel B. Thomas ${ }^{1,2}$, Amelia Adcock ${ }^{3,4}$, and Valeriya Gritsenko ${ }^{1,2}$

\section{Affiliations:}

${ }^{1}$ Department of Human Performance, Division of Physical Therapy, School of Medicine West Virginia University, Morgantown, WV, 26506

${ }^{2}$ Rockefeller Neuroscience Institute, Department of Neuroscience, West Virginia University, Morgantown, WV, 26506

${ }^{3}$ WVU Center for Teleneurology and Telestroke, Morgantown, WV, 26506

${ }^{4}$ Department of Neurology, School of Medicine, West Virginia University, Morgantown, WV, 26506

Corresponding author: Valeriya Gritsenko, PO Box 9226, One Medical Center Drive, Morgantown, WV, 26505-9226. Phone: (304) 293-7719

Running Head: dynamic assessment of post-stroke reaching

Supplementary Figures are available at https://github.com/arielthomas/Thesis-Chapter-4Supplementary-Figures/releases/tag/Fig1 


\section{Rationale}

The goal of an assistive device in a stroke survivor is to restore a base level of movement which allows them to confidently perform everyday tasks. Functional electrical stimulation (FES) can activate shoulder muscles to create movement without descending neural commands, or can be used assistively during movement to increase muscle force. Various wearable neuroprosthetics for grasp restoration using Functional Electrical Stimulation (FES) have demonstrated rehabilitative success in acute and chronic stroke patients(Micera et al. 2010; Niu et al. 2019; Sun et al. 2018). Positive results have also been seen for elbow extension (Hughes et al. 2010; Thrasher et al. 2008), shoulder motion (Hara et al. 2008), and stabilization of wrist joints (Malešević et al. 2012). However, in clinical studies that have examined the therapeutic effects of shoulder stimulation on movement, a disproportionately large number have focused on cyclic stimulation, or cyclic neuromuscular electrical stimulation (NMES), with patterns that are not coordinated with ongoing movement. For example, numerous papers (Church et al. 2006; Kobayashi et al. 1999; Linn et al. 1999; Mangold et al. 2009; Wang et al. 2002) have stimulated the posterior deltoid while ignoring the medial and anterior deltoid, which would strengthen muscles to increase external rotation and abduction of the shoulder, but is unnatural for shoulder extension. Not surprisingly from a synergistic or biomechanical perspective, these studies have not shown significant long-term improvement for reach tasks which rely primarily on shoulder flexion. While these and similar papers used to be commonly referenced to disprove the effectiveness of FES to restore movement performance after stroke in the past (Knutson et al. 2018; Vafadar et al. 2015), more recent studies have been sure to incorporate neuroscience and biomechanically-informed strategies into FES application (Amano et al. 2020; Chou et al. 2020; Niu et al. 2019; Smith et al. 2019; Sousa et al. 2021). It is an imperative to the field to create a stimulation protocol that makes sense in the context of functional tasks, as these are likely to be the most effective at inducing beneficial neuroplastic changes. It has also been seen that user-driven, biomechanically inspired FES approaches are not too complicated to be incorporated into clinical practice, as therapists from a wide range of backgrounds have been able to use versions of these systems without on-site technical support (Smith et al. 2019). Perhaps most importantly, stroke survivors have responded to these approaches very favorably (Smith et al. 2019).

Our FES approach combines gravity unloading with biomimetic principles of muscle recruitment, which both have been seen to be effective independently in lab settings to improve reaching performance. Using an assistive robotic device to passively reduce the load of gravity on the 
shoulder during reaching and grasping has already been shown to improve muscle coordination for grasp, potentially by decoupling the neural activity at the shoulder from the rest of the arm (Lan et al. 2017). While several studies have used a robotic support system, uncoupling neural activity at the shoulder from the hand using antigravity functional electrical stimulation (FES) support of shoulder muscles has not been tested. Here, we are using shoulder FES to reduce the effort needed to lift the arm against gravity with the goal of improving reach and grasp performance.

\section{Methods}

\section{Subjects}

For the calculation of gravity dependent muscle torque, we used data collected from the control participants in Olesh et al., 2017 and Thomas et al., 2021 (Described in Chapter 2). For the FES Task, we recruited 4 stroke survivors (aged $50 \pm 6$ ) with right hemisphere strokes and a range of hemiparetic symptoms: (1) Moderate impairment (NIH Stroke Scale $=3$ ) with flaccid hemiparesis, (2) Moderate impairment with overactive flexor synergies, (3) Severe impairment (NIH Stroke Scale $=6$ ) with flaccid hemiparesis, (4) Severe impairment with overactive flexor synergies $(\mathrm{NIH}$ Stroke Scale $=6)($ Table 1$)$. Exclusion criteria included the inability to produce visible movement with their shoulder and elbow or the inability to provide written consent to participate. We also recruited 20 control participants (aged $24 \pm 3$ years, 14 Female, 8 Male) with no neuropathological motor conditions to provide a baseline of non-impaired movement for the FES task (Supplemental Table 1) Control subjects were excluded if they had any neurological conditions that could affect their motor control. Of the 20 controls, all but one participant was right hand dominant (C11), who had their dominant left hand tested. Subject C6 was tested on their right, non-dominant hand. One control (C1) and one stroke subject (S2) participated in an additional data collection on a separate day to assess cyclic FES applied during the task to replace triggered FES. However, this additional cyclic data was not analyzed in this study. The study and the consent procedure were approved by the Institutional Review Board of West Virginia University (Protocol \# 19065977769). Subjects provided written consent before participation.

Table 1: FES Reaching Task Stroke Participant Information

\begin{tabular}{|c|c|c|c|c|c|c|c|c|}
\hline Subject & $\begin{array}{c}\text { Age } \\
\text { (year) }\end{array}$ & Sex & $\begin{array}{c}\text { Dominant } \\
\text { Hand }\end{array}$ & $\begin{array}{l}\text { Hand } \\
\text { Tested }\end{array}$ & $\begin{array}{l}\text { Stimulation } \\
\text { Amplitude }\end{array}$ & $\begin{array}{c}\text { Stimulation } \\
\text { Type }\end{array}$ & Protocol & Stroke Description \\
\hline
\end{tabular}




\begin{tabular}{|c|c|c|c|c|c|c|c|c|}
\hline & & & & & $(\mathrm{m})$ & & & \\
\hline S1 & 43 & $M$ & $\mathrm{R}$ & $\mathrm{L}$ & $62 / 63$ & Triggered & A & $\begin{array}{l}\text { Right MCA Stroke, } 7 \text { months post-stroke, NIH SS } \\
=3\end{array}$ \\
\hline S2 & 57 & $M$ & $\mathrm{R}$ & $\mathrm{L}$ & $\begin{array}{l}22 / 22 \\
30 / 35\end{array}$ & $\begin{array}{l}\text { Cyclic and } \\
\text { Triggered }\end{array}$ & A & $\begin{array}{l}\text { Right Lacunar Stroke, } 12 \text { Years Post-Stroke, NIH } \\
\mathrm{SS}=3\end{array}$ \\
\hline S3 & 47 & $M$ & $\mathrm{R}$ & $\mathrm{L}$ & $37 / 45$ & Cyclic & A & $\begin{array}{l}\text { Right MCA Stroke, } 8 \text { Years Post-Stroke, NIH SS = } \\
6\end{array}$ \\
\hline S4 & 51 & $M$ & $R$ & $\mathrm{~L}$ & $30 / 35$ & Cyclic & A & $\begin{array}{l}\text { Right MCA Stroke, } 5 \text { Years Post-Stroke, NIH SS = } \\
6\end{array}$ \\
\hline
\end{tabular}

\section{Calculating Gravitational Component of Muscle Torque}

The slowly changing, or static component of muscle activity is likely to support the arm against gravity. To test this idea, we used inverse dynamic simulations to calculate the gravity-related component of active joint torques during reaching movements (Olesh et al., 2017). The 1st principal component of gravity-related muscle torque evolves similarly to the 1st principal component of EMG during reaching movements, and it captures a lot of variance across multiple muscles. This is especially true for the anterior deltoid (AD), in which $80 \%$ of variance was explained by the gravitational component of torque across 28 different movement directions (Olesh et al., 2017). We used this profile (Fig. 1) to design a pattern of shoulder muscle stimulation that would offset the gravity load during a reaching movement, termed gravity-assist FES. For the forward reach, we applied the gravitational dependent profile of active muscle torque for the flexion/extension degree-of-freedom as stimulation to the AD. For the side (lateral) reach, we applied the gravitational dependent profile of active muscle torque for the abduction/adduction degree-of-freedom as stimulation to the MD. These muscles play a major role in movement in these directions (Table 2). 


\section{Gravitational Muscle Torque}

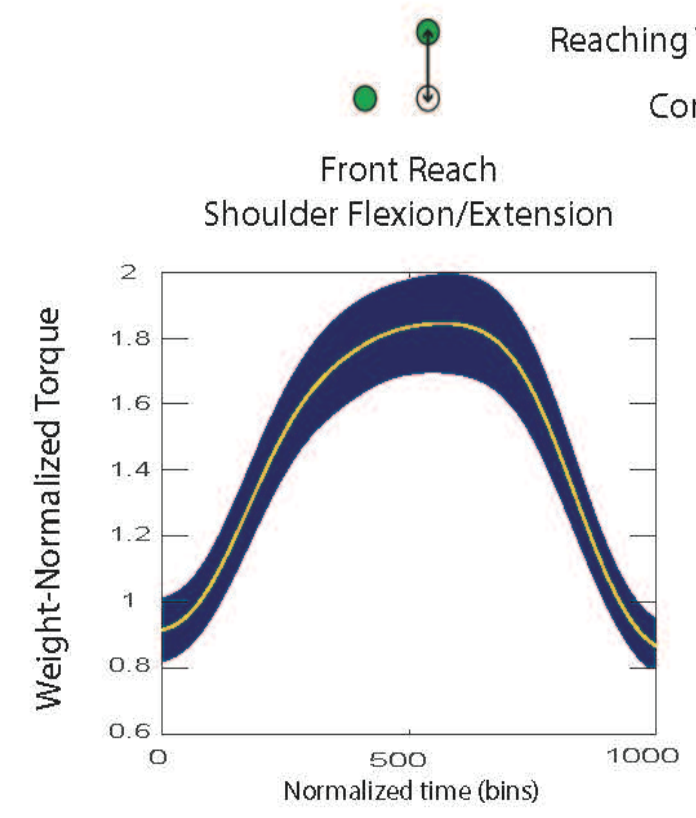

Controls $(n=9)$

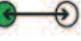

Front Reach

houlder Flexion/Extension

Side Reach

Shoulder Abduction/Adduction

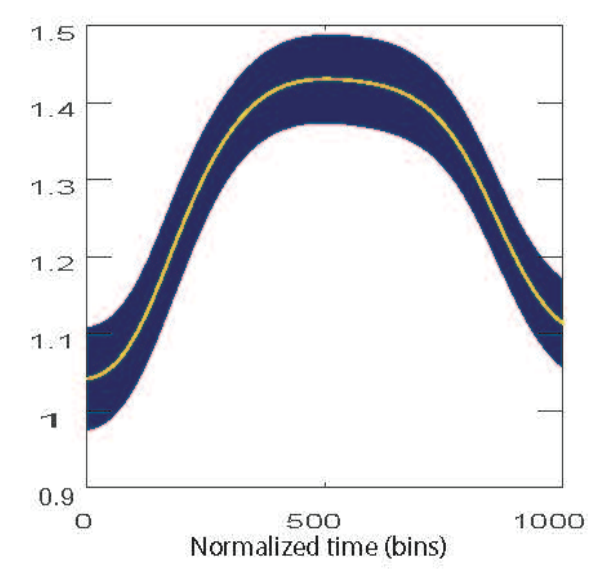

Figure 1. Gravitational Muscle Torque Profiles used to design Gravity Support FES Parameters. Profile of gravity-dependent muscle torque from 9 young control subjects, calculated in chapter 2. Displayed are the profiles, averaged across 15 center-out and return movements, normalized by arm weight, and averaged across subjects. Mean is shown in gold with standard error in blue. Target location to the front or side targets (targets 5 and 7 in chapter 2), are the same location as those given in the FES task. However, no grasping on the target was required during this 'pointing' reach task. Additionally, no feedback on speed was provided during this pointing task.

\section{Using FES for Anti-Gravity Support During Reach and Grasping Task}

Subjects performed a reaching and grasping task in a virtual reality (VR) environment (Unreal Engine 4) with detailed visual feedback of hand position using a camera-based motion capture system (LEAP Motion) mounted onto the VR headset (VIVE). Using the data captured by LEAP Motion, both the appearance of targets and onset of stimulation was triggered by the subject's interaction with virtual targets (2cm radius ball). Within the Unreal Game, we used a proximity sensor that triggers stimulation based on hand location relative to target location, with a secondary statement that required distal finger and thumb markers to be in close proximity when at the location of the target. This did not require the hand to reopen in order for the automated system to continue. This system was designed because of the difficulty that stroke participants have with hand opening. All subjects were observed to make sure that they are performing grasps on 
targets, and not moving with a closed hand. Hand aperture profiles show that in all control subjects, the hand was opening and closing at regular intervals.

Subjects are provided an initial starting target at 90 degrees elbow flexion and 0 degrees shoulder flexion. Distal targets appeared at $25 \%$ of the subject's arm length from the initial target. These targets were chosen because they are commonly recruited for functional reaching tasks and have unique patterns of shoulder movement. The forward target ( $0^{\circ}$ horizontal plane) primarily required shoulder extension, while the lateral target $\left(90^{\circ}\right.$ horizontal plane) required shoulder abduction and/or external rotation. Both targets required the subject to keep their arm elevated against gravity.

Pre-stimulation control trials and stimulation amplitude fitting were conducted prior to stimulation trials. We assigned a subject-specific fixed stimulation amplitude for each target movement, based on minimum frequency ramps $(2 \mathrm{~s}, 0-30 \mathrm{~Hz})$ at that amplitude required to cause voluntary movement of 20 degrees shoulder flexion (anterior deltoid) or shoulder abduction (medial deltoid) when the arm was relaxed. These stimulations and their effect on shoulder movement during rest were repeated 10 times per stimulation type to examine variability in stimulated muscle activity. Stimulation amplitudes per subject are reported in table 1 , and the induced movement is reported in Figure 4E. During stimulation trials, FES was applied to the shoulder in frequency ramp sequences $(0-15 \mathrm{~Hz})$ in which the fixed amplitude to recreate the flexion gravitational muscle torque profiles for of forward and return front reaching movements and and abduction gravitational muscle torque profiles for forward and return side reaching movements. Stimulation reached $30 \mathrm{~Hz}$ after 2 seconds and remained at $30 \mathrm{~Hz}$ for a maximum of 3 seconds or until the subject correctly reached to and grasped the next target. Target interaction and grasping events were recognized automatically using LEAP motion tracking of the hand. Once the correct reach and grasp occurred, the center target would reappear, and the ramp sequence would decrease back to $0 \mathrm{~Hz}$ over 2 seconds for the return movement. At the end of the experiment, we repeated the resting stimulation pulses and collected post-stimulation control trials to test for fatigue and changes in movement.

Kinematics of the index finger, thumb, wrist, arm, and torso were captured using an active LED motion capture system (Phasespace Impulse). Additional kinematics of the hand, including all 5 digits and target interaction events, were recorded using LEAP Motion. Over the course of the experiment, some repetitions were excluded due to occluded markers. Stroke participants 3 and 4 were unable to extend their fingers out of a closed fist, and therefore LEAP could not detect their hand. For these participants, we instructed the task without virtual reality. Targets (tip of a 
pen or stick) were positioned in the room at the distance and position equivalent to those in the virtual reality task, and the participants were instructed to reach and attempt to grasp them. FES was provided through cyclic stimulation through a portable TENS unit $(35 \mathrm{~Hz}, 200 \mu$ s pulsewidth, 1s ramp up, 2s on, $1 \mathrm{~s}$ ramp down, off $2 \mathrm{~s}$ ).

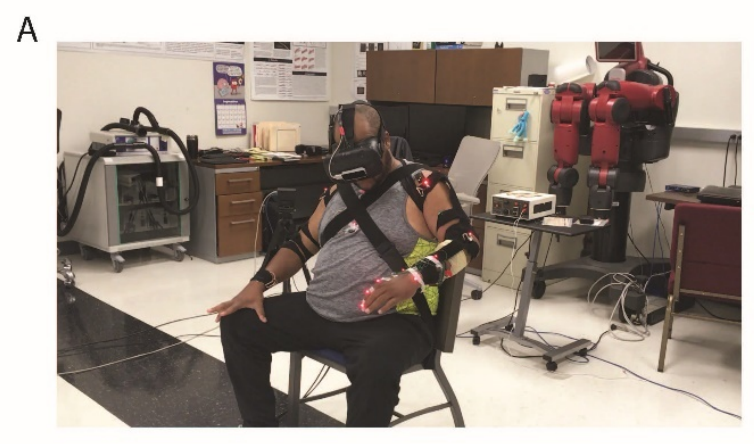

B

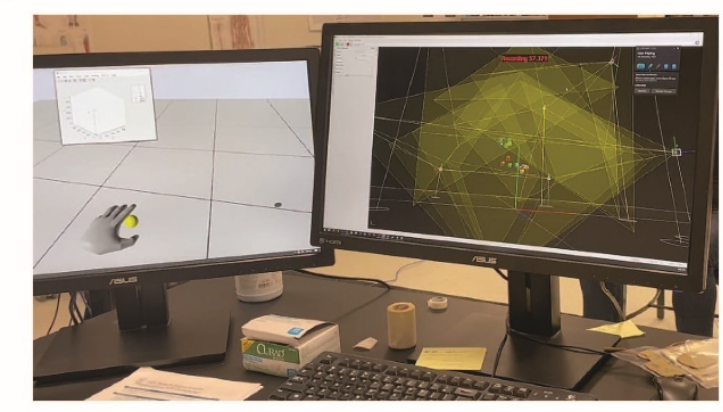

C
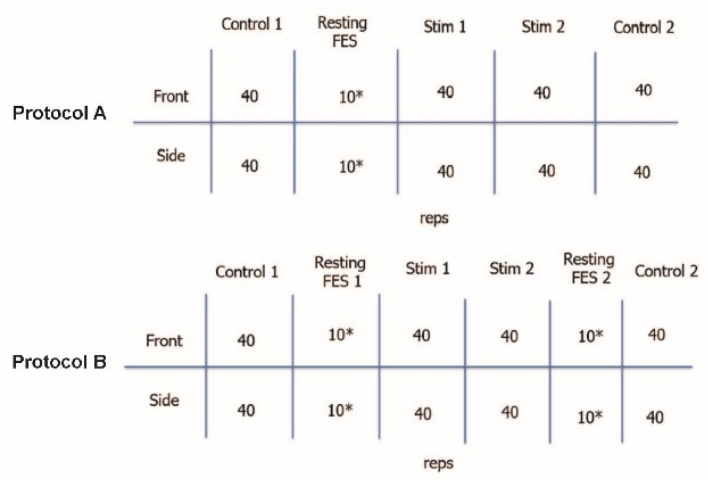

$*=$ No reaching task
D

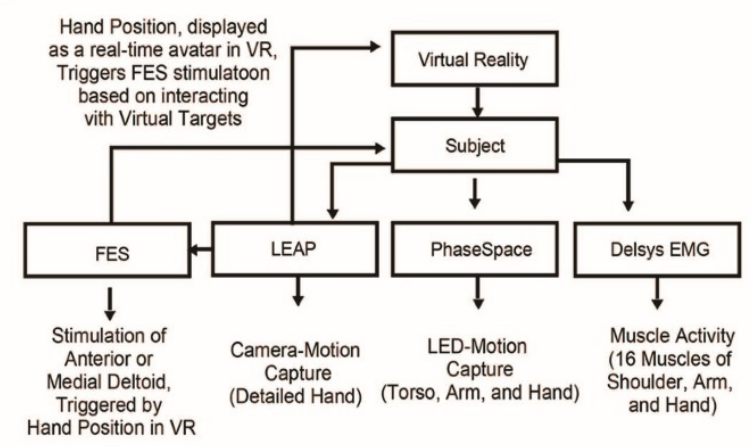

$E$

Passive Stimulation (no task)

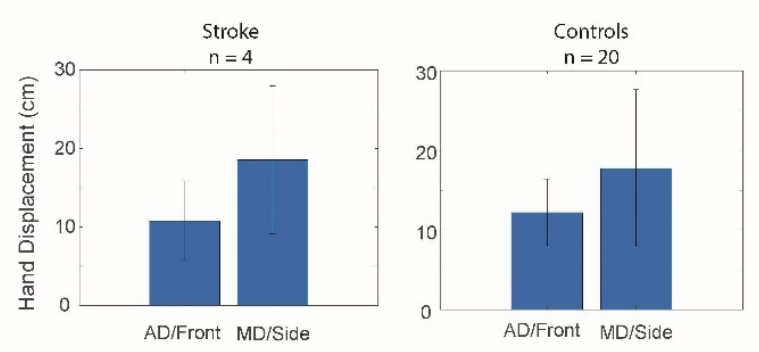

$\mathrm{F}$

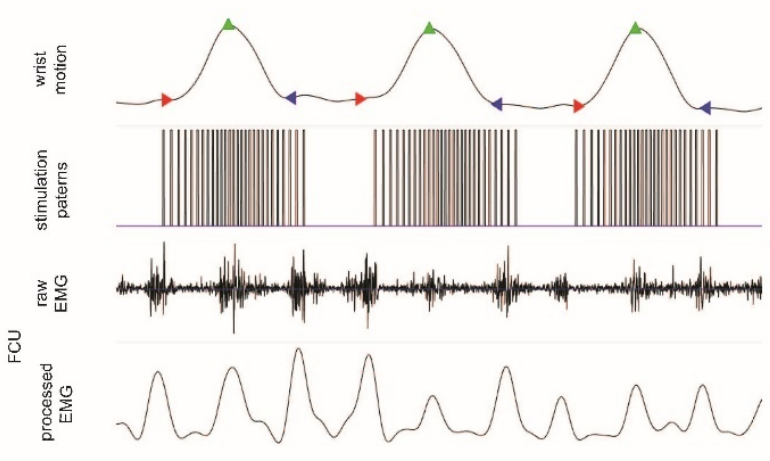

time (s)

Figure 2. Functional electrical stimulation of the shoulder during reaching. A. Subjects were seated and asked to perform reach and grasp tasks in an immersive virtual reality environment. $\boldsymbol{B}$. Virtual Environment (left) shows targets and a virtual representation of real-time subject hand position (LEAP Motion). Phasespace motion capture (right) was collected for the torso, arm, and hand. C. Subjects were given either 
protocol A or protocol B. Protocol B was 10 minutes longer and included a second passive stimulation session ('Resting FES 2') after stimulation trials but before the second set of control trials. $\boldsymbol{D}$. Schematic illustrating how subject data is collected and used to inform task. E. Movement induced by FES parameters during rest. FES was applied to the medial or anterior heads of deltoid muscles with ramp frequency sequences to recreate gravitational muscle torque profiles of the corresponding movements. Amplitude of stimulation was customized to the subject and set for the remainder of the experiment. F. Example profiles of kinematics (wrist motion), stimulation pulses (recorded by National Instruments), unfiltered FCU EMG, and processed FCU EMG.

Electromyography (EMG) of 16 muscles of the arm and hand (Table 2) were captured using a wireless surface EMG system (DELSYS). For one control subject different thumb muscles were recorded (APL and EPB), and therefore this subject's thumb EMG was excluded from analysis. During stim trials, EMG electrodes on the $A D, M D$, and PD were removed, but replaced after stimulation trials were over. Replacement was accurate due to the alignment of the electrode with small indentations in the skin caused by the pressure of the device. Collected EMG was proccessed in Matlab with custom scripts. EMG was filtered to remove stimulation artifacts by detecting stimulation artifacts peaks above the $54 \%-62 \%$ standard deviation of the data in either biceps or triceps, and removing emg in a $200 \mu$ s window centered on the peak, and replacing it with signal mean. This was applied in all muscles where stimulation artifacts were detected (confirmed with waveform detection and visual inspection). Stimulation artifacts were visible in distal muscles for two stroke subjects, due to high stimulation amplitide, and stimulation pulses were removed. EMG was high pass filtered with a $20 \mathrm{hz} 2^{\text {nd }}$ order butterworth filter, rectified, demeaned, and low pass filtered with a $5 \mathrm{hz} 4^{\text {th }}$ order butterworth filter to create an EMG envelope. To identify 'ideal' EMG patterns for each muscle to compare to stroke, EMG for the control nonstim trials was averaged across repetition within each condition, normalized by the maximum mean EMG across all conditions, and then averaged across subjects. Signal variability for EMG was small across repetitions but larger across subjects. For each stroke participant, EMG for 6 muscles of the hand was normalized by the maximum mean EMG across all conditions, and compared using RMSE to the ideal control signal profile for each reach. This included one flexor and one extensor muscle for the wrist, fingers, and thumb

\section{Table 2. Muscle Descriptions}

\begin{tabular}{|l|l|l|}
\hline Abbreviation & Full Muscle Name & Muscle Action \\
\hline Pec & Pectoralis major & shoulder flexion, adduction and internal rotation
\end{tabular}




\begin{tabular}{|c|c|c|}
\hline$A D$ & Anterior Deltoid & $\begin{array}{l}\text { shoulder flexion, abduction, and internal } \\
\text { rotation }\end{array}$ \\
\hline MD & Medial Deltoid & shoulder flexion and abduction \\
\hline PD & Posterior Deltoid & $\begin{array}{l}\text { shoulder extension, abduction, and external } \\
\text { rotation }\end{array}$ \\
\hline InfraSpin & Infraspinatus & houlder external rotation and stability \\
\hline SupraSpin & Supraspinatus & shoulder abduction and stability \\
\hline BicL & Biceps Brachii (long head) & shoulder flexion, elbow flexion \\
\hline TriLo & Triceps Brachii (long head) & shoulder extension, elbow extension \\
\hline $\mathrm{Br}$ & Brachioradialis & elbow flexion \\
\hline PTER & Pronator Teres & forearm/wrist pronation \\
\hline FCU & Flexor Carpi Ulnaris & wrist flexion \\
\hline ECU & Extensor Carpi Ulnaris & wrist extension \\
\hline FDS2 & $\begin{array}{l}\text { Flexor Digitorum } \\
\text { Superficialis }\left(2^{\text {nd }}\right. \\
\text { Compartment) }\end{array}$ & finger flexion \\
\hline ED2 & $\begin{array}{l}\text { Extensor Digitorum } \quad\left(2^{\text {nd }}\right. \\
\text { Compartment })\end{array}$ & finger extension \\
\hline FPL & Flexor Pollicis Longus & thumb flexion \\
\hline EPL & Extensor Pollicis Longus & thumb extension \\
\hline
\end{tabular}

Hand aperture, measured as the distance between the tip of the index finger and tip of thumb, was examined for each trial. Hand kinematics were obtained using both an LED-based syatem and a camera based system (LEAP) for all controls and the two mild stroke subjects. However, LEAP was not detected reliably for the two moderately affected stroke subjects due to their difficulty with hand opening and was was therefore not recorded. Measures were consistent between the two systems; however due to two stroke subjects not having LEAP motion capture's measurements, we used phasespace for hand arperture measures in 18/20 control subjects and all 4 stroke subjects. In 2 of the controls, phasespace leds of the fingertip or thumb were occluded from detection during grasping, and therefore LEAP motion capture was used. Variability in hand aperture was small both across repetitions and across subjects in control subjects (Fig. 5). To get a value to compare with stroke subjects, peak to peak arperture of control subjects was averaged across repetitions within each condition and then averaged across subjects. For each stroke 
participant who was able to grasp, the hand aperture was compared to controls between stimulation and non-stimulation trials. A second set of experiments were added to this study. Protocol B was added to this experiment for 9 control subjects in order to examine changes in the movement amplitude induced by FES stimulation after using this with a task for an extended period of time.

\section{$\underline{\text { Results }}$}

FES was capable of assisting with shoulder abduction and flexion and did not interfere with the execution of reaching movements (Fig. 3). The FES assistance was maintained throughout the multiple repetitions of reaching movements over a 40-minute period, which suggests that muscle fatigue can be managed with FES. Task completion was also faster during stimulation trials in both stroke subjects with self-triggered FES (Fig.4). Of note, stroke subjects 3 and 4 had cyclic stimulation, and therefore speed was partially modulated by the stimulation parameters. Grasping performance was improved in the stroke subject during stimulation trials. Only the two least impaired stroke participants were able to reliably open and close their hands without stimulation, and in these two subjects grasping coordination was improved with FES (Fig. 5). Improved coordination was assessed as its similarity to the average profile of controls. Control subjects individually had small variability in these profiles across repetitions (Fig. 6A), however there was larger variability across subjects (Fig 6B), especially in the forward reach. FES had no effect on hand aperture in control participants for the side reach, but in forward trials the hand appears to open earlier in the movement with FES than without FES. 
Stroke Participant 1 $\mathrm{NIHSS}=3$
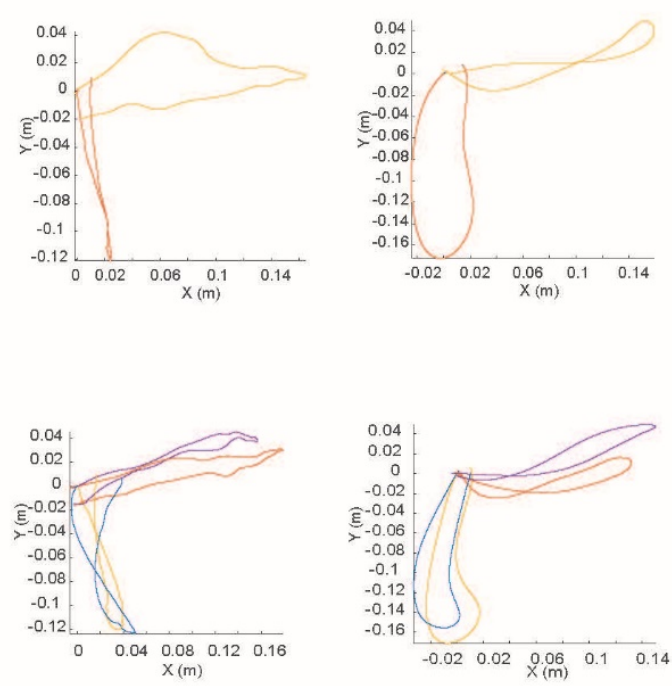

Endpoint Trajectory

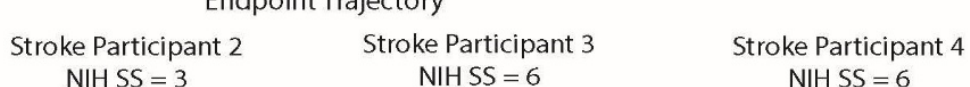

Stim Trials
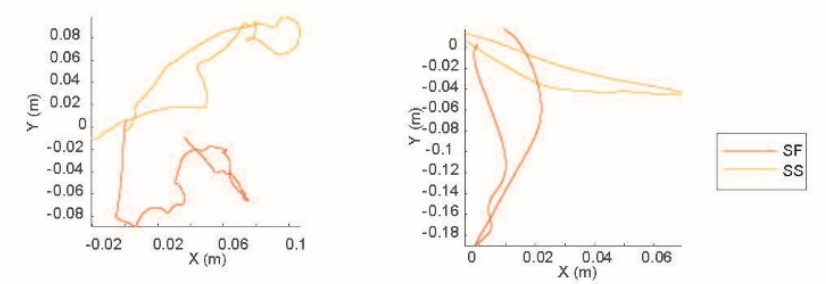

Control Trials
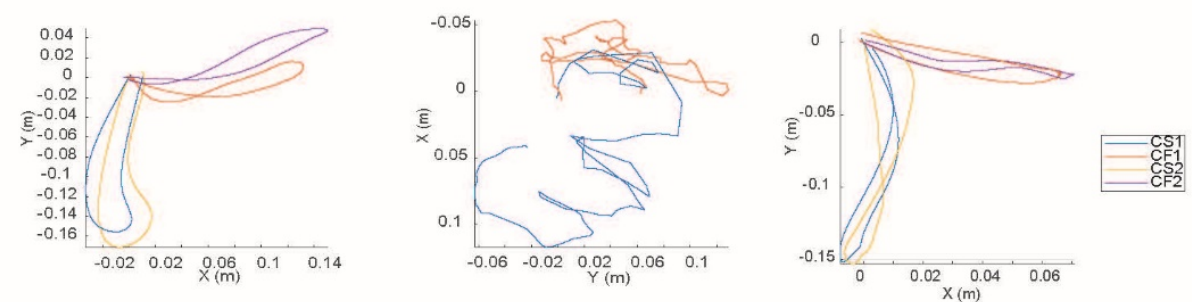

Figure 3. Reach Trajectories. Endpoint trajectories are similar with and without FES. Shown: Forward and Lateral Center-out and return endpoint trajectories averaged across trial type for stimulation and control trials for each stroke participant. Top row displays Stim Front (SF, gold trace) and Stim Side (SS, orange trace). Bottom row show Control Trials prior to Stim Trials: Control Side 1 (CS1, blue trace) and Control Front 1(CF1, orange trace), along with Control Trials after Stim Trials: Control Side 2 (CS2, gold trace) and Control Front 2 (CF2, purple trace).
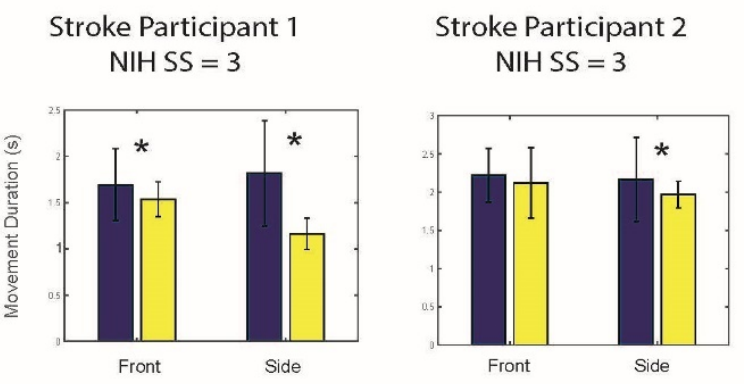
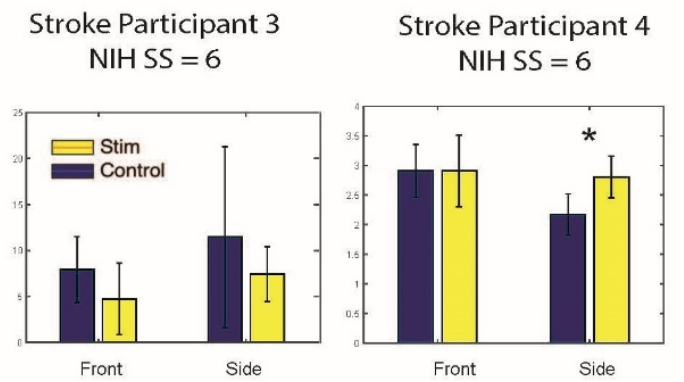

Figure 4. Movement Duration compared between Stimulation (gold) and Control (blue) task trials. Data shown for each center-out and return movement repetition. Control trials before and after stimulation are both included. Error bars show standard deviation across repetitions. Two-sample t-tests were performed between conditions, asterisks represent $p$-values $<0.05$. Supplemental statistics are provided below. 


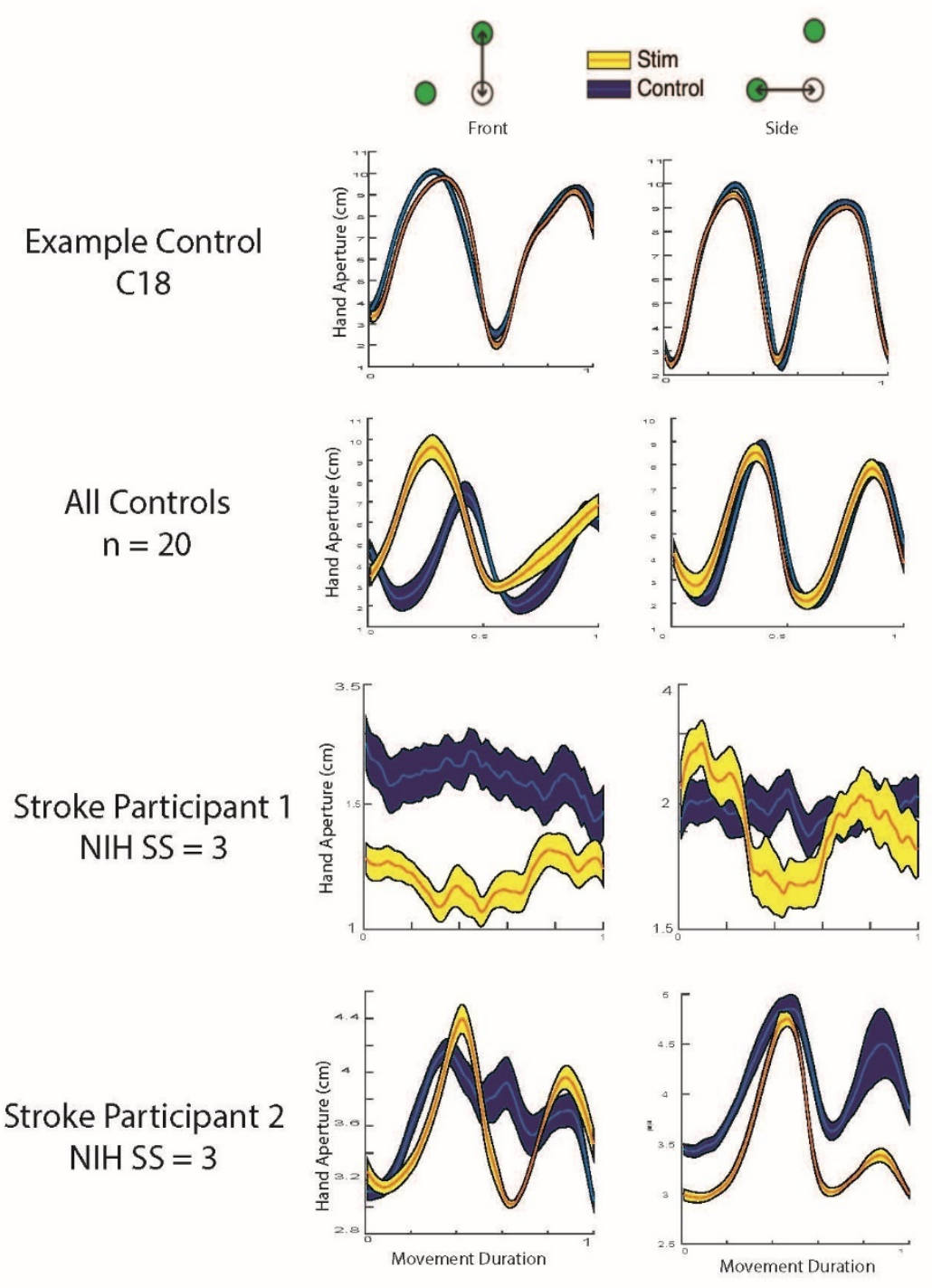

Figure 5. Grasping Performance. Grasping ability compared as Hand Aperture profile across time, between Stimulation (gold) and Control (blue) task trials. Hand aperture was calculated as the distance between tip of finger and thumb tracked by Phasespace Motion capture, across lateral reaching stimulation and control trials, averaged by condition. In the two least impaired stroke subjects, grasping performance was modulated by FES to appear more similar to controls. 

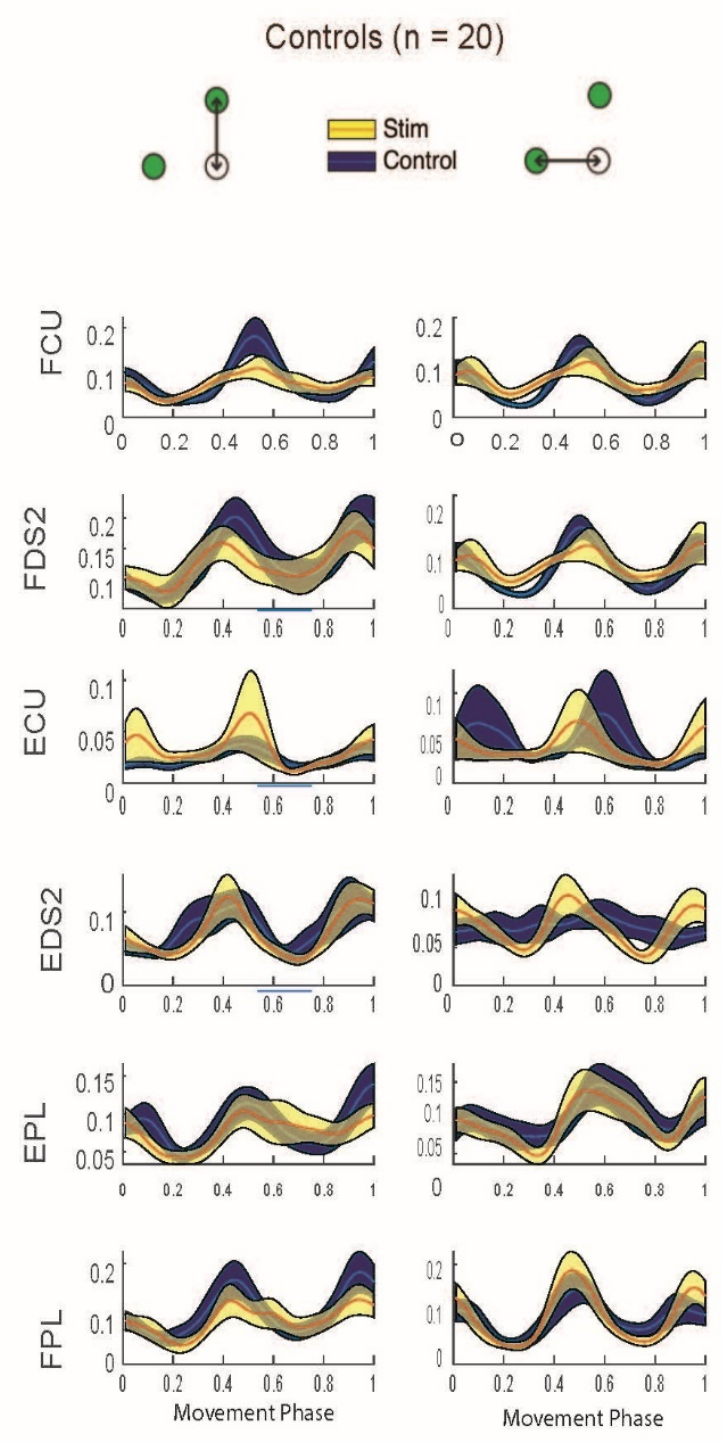

Figure 6. Control Muscle Activity (EMG). FES had minimal modulatory effects on distal muscles not directly simulated in control participants. In the control participant, muscle bursts in FCU are diminished by FES while muscle bursts are increased in EDS2. Shown: filtered, normalized EMG activity averaged across forward reaching stimulation and control trials, averaged per block (40 repetitions). Wrist actuators: Flexor Carpi Ulnaris (FCU), Extensor Carpi Ulnaris (ECU). Finger Actuators: Second Compartment of Flexor Digitorum Superficialis (FDS2), Second Compartment of Extensor Digitorum (ED2). Thumb Actuators: Extensor Pollicis Longus and Flexor Pollicis Longus. 
Distal muscle coordination was also improved in stroke participants using shoulder FES stimulation. Control EMG had stereotypical phasic activity of flexors and extensors that drives the hand aperture (Fig. 7). Stimulation trials in controls had decreased FCU peaks and increased EDS2 peaks, but no other overall changes to distal EMG. Stimulation trials in stroke participants had EMG patterns that were more similar to control non-stim EMG patterns (nonstim) than without stimulation (Fig. 6). We examined this further using an RMSE analysis comparing control non-stimulation EMG and stroke EMG for both conditions (Fig. 7). Control trials had larger RMSE values than Stim Trials for most muscles in most conditions. One exception is the side trials for stroke subject 1 , in which stimulation trials had significantly larger RMSE values for FDS2 and ECU, although they were significantly smaller for ED2 and had no significant difference for the other muscles. The largest differences were seen for the extensor muscles of the fingers and thumb (ED2 and EPL). We incorporated Protocol $B$ for nine of the healthy subjects to examine changes in movement induced by FES over time (Table 1, Figure 4C). We found a significant decrease in the amount of movement generated by stimulation after assisted trials, indicating muscle fatigue (Fig. 9). These results could be indicative of a potential hurdle in wearable FES technology. Because of this, we invited stroke participants 2 and 3 back to the lab to evaluate the adjustments to amplitude necessary to maintain the same movement amplitude over time (discussed in Chapter 4). More research will need to be done on the effectiveness of this method but there could be a potential need to adjust amplitude of the FES during extended use 

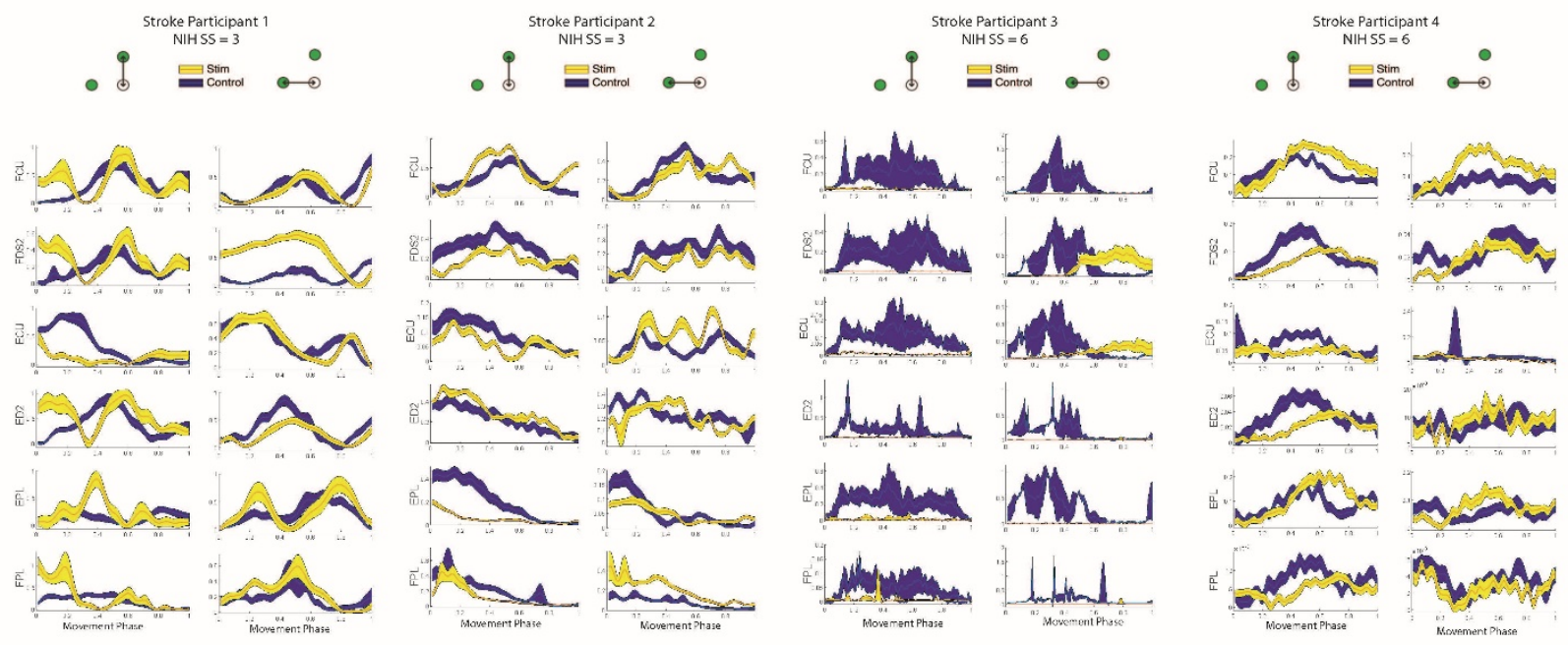

Figure 7. Stroke Muscle Activity (EMG). FES had modulatory effects on distal muscles not directly simulated in stroke participants. In the stroke participants, most distal hand muscles showed increased modulation. Shown: filtered, normalized EMG activity averaged across forward reaching stimulation and control trials, averaged per block (40 repetitions). Wrist actuators: Flexor Carpi Ulnaris (FCU), Extensor Carpi Ulnaris (ECU). Finger Actuators: Second Compartment of Extensor Digitorum (ED2). Thumb Actuators: Extensor Pollicis Longus and Flexor Pollicis Longus.

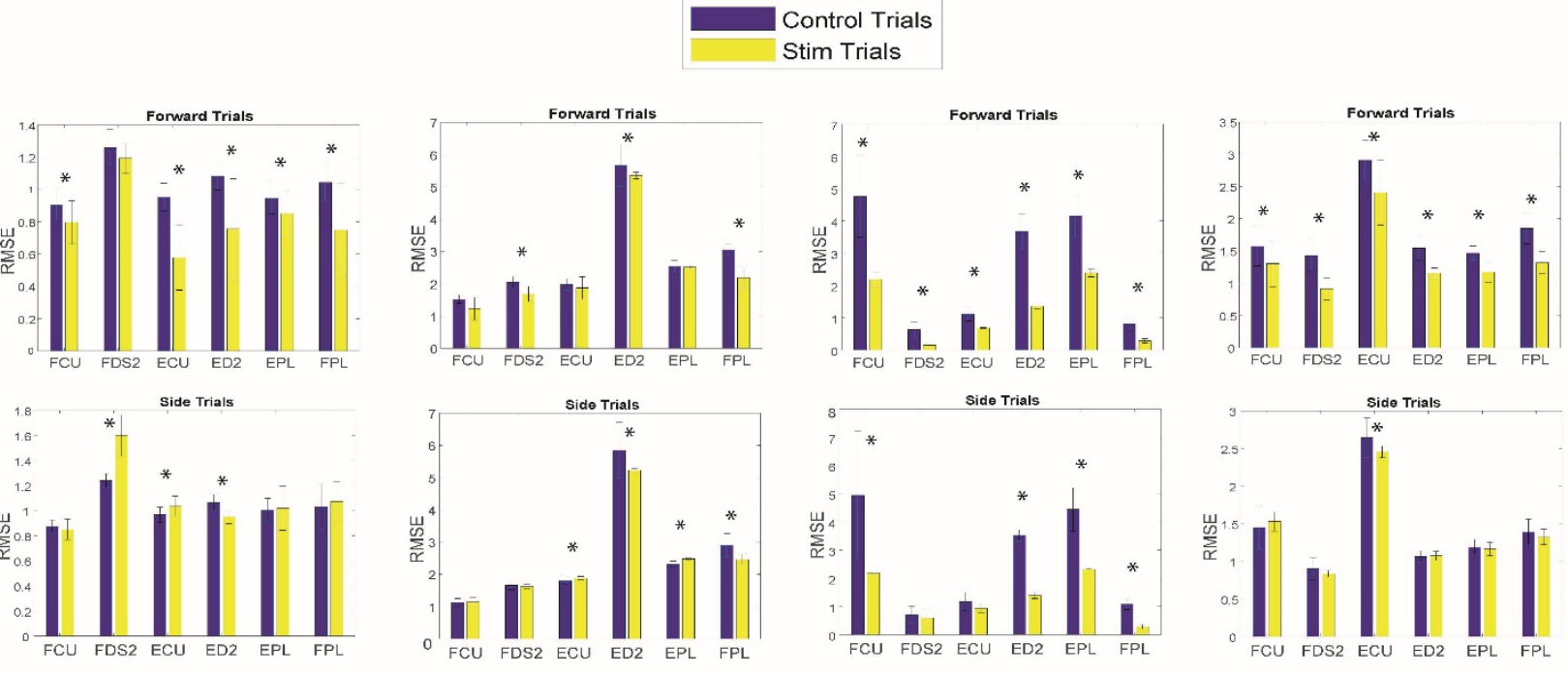

Figure 8. Muscle activity for the hand compared between Stimulation (gold) and Control (blue) task trials. Data shown for each center-out and return movement repetition. Control trials before and after stimulation are both included. Error bars show standard deviation across repetitions. Two-sample t-tests were 
performed between conditions, asterisks represent $p$-values < adjusted $\alpha$ : 0.00833 . Supplemental statistics are provided below.

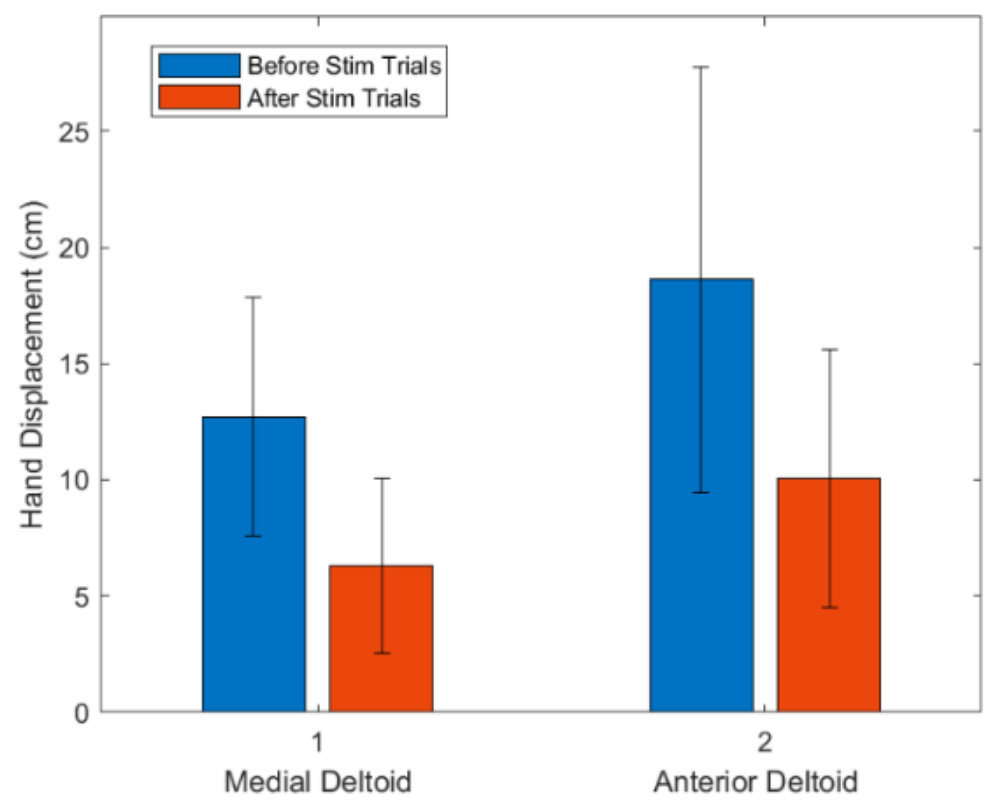

Figure 9. Stimulation Fatigue. Hand Displacement $(\mathrm{cm})$ was averaged across 10 repetitions of each condition, and then compared across 9 subjects. Error bars show standard deviation across subjects. Paired t-tests comparing Before and After conditions found hand displacement caused by passive stimulation to be significantly different for both Medial Deltoid and Anterior Deltoid Stimulation ( $p=0.004$, and $p=0.015$ respectively). This shows a significant change in hand displacement caused by passive stimulation after the stimulation task trials.

\section{Discussion}

A variety of rehabilitation techniques and assistive technologies are being studied in hopes of finding more successful ways to improve functionality and the quality of life of stroke survivors. One example of a common rehabilitation technique is functional electrical stimulation (FES) which is the artificial stimulation of muscles that causes muscular contractions sufficient to produce or assist with movement. Here I have shown that it is feasible to use shoulder FES to support the arm against gravity during reaching. I have also shown that the hand muscle activity of stroke survivors decreases, which suggests a potential use of shoulder FES for reducing hand spasticity. 
These EMG results here are similar to those seen when using gravitational support of the abduction load on forward reaches (Lan et al. 2017). Grasping ability (hand aperture), muscle activity, and movement duration of these two stroke subjects were analyzed in FES conditions and non-FES conditions. I have also shown that shoulder FES increases movement speed and hand opening of stroke survivors, which has the possibility of improving reaching and grasping function. However, the reach trajectory of the stroke subjects was not affected. This study is limited by the small sample of stroke participants. No across subject comparisons could be made, and we are unable to make inferences to the general population. However, we see this as a positive feasibility study to show the potential for this method.

FES has some inherent limitations, such as insufficient selective activation of muscles and muscle fatigue (Pelton et al. 2012). The negative effect of muscle fatigue induced by FES is temporary but should be improved to maintain the safety and practicality of the device. Techniques for the alleviation of muscle fatigue during electrical stimulation include improving electrical stimulator control parameters and improving upon the placement and design of FES electrodes. The first option we have already implemented, as we have incorporated a step to adjust the amplitude of stimulation and position of electrodes for best muscle contraction with least stimulation and discomfort. Our preliminary data showed no problem with fatigue; however, we may examine alternative stimulation device strategies in the future. Surface electrode arrays, first used to activate forearm muscles (Nathan 1979), provide less fatiguing and more selective The average distance of passive stimulation hand displacement before and after stimulation trials was measured in centimeters and then plotted on a bar graph. The displacement distance was significantly lower than before the trials. This could indicate fatigue from the stimulation.

Average hand displacement caused by passive stimulation muscle activation than single electrode pair applications. In addition, the ability to independently control each electrode in the array gives the ability to optimize the activation of specific motor units, thereby improving the ability to generate a specific target movement. Our lab has a grid stimulation device (RIPPLE) that could be programmed to selectively apply anterior and medial deltoid stimulation. Fatigue generated over time will evaluated in greater detail in Chapter 4.

While our model offers 'predictive' stimulation only in conjunction with VR target assignment, there are options for predictive stimulation control using movement tracking with accelerometer data. Two labs have recently developed independent predictive control strategies for FES support of the moderately motor impaired arm using accelerometry data (Klauer et al. 2016; Sun et al. 2016). The Schauer lab developed a control strategy for the amplification of volitional shoulder abductions through FES and showed an effective prevention of fatigue as 
well as arm weight support (Klauer et al. 2016). FES-induced muscle recruitment in this study was generated based on the abduction angle of the arm. Feedback control was provided through their designed recruitment controller, which included a user-optimized filtering algorithm of the FES-evoked EMG to provide the amount of FES stimulation that promotes effective movement without fatiguing the muscle. They then applied antagonist muscle co-contraction to the anterior and posterior deltoid to support the arm, and found that this increased movement stability, but also accelerated muscle fatigue. The Smith lab (Sun et al. 2016) applied stimulation to both muscles of the shoulder and elbow in order to create desired joint angles for functional tasks with a predictive algorithm that identified the task. Our method would employ strategies similar to either lab, with the alternative use of gravitational torque profiles selected by feedback from arm position. Our EMG recording electrodes (DELSYS) contain accelerometers, and could be used to find the extension and abduction angles of the arm for use with amplification of gravity torque stimulation.

Wearable FES devices are lightweight and affordable, making them preferential over robotic devices. Therefore, we suggest that assistive FES to provide gravity support on the shoulder could be used to improve grasping function of stroke survivors. Future works could be conducted to develop a portable, wearable device to apply triggered FES to the shoulder of stroke survivors in hopes of improving hand performance. 
Chapter 4: Examining Fatigue and the impact on the Glenohumeral Joint for Shoulder FES

Some text and figures adapted from NCM poster: The Fatigue Caused By Functional Electrical Stimulation Of Deltoid Muscle Does Not Reduce Its effect on The Glenohumeral Distance by Rawan AIGhawi, Ariel B. Thomas, Cheryl L. Brandmeir, Yu-Jen Chang, Sergiy Yakovenko, and Valeriya Gritsenko

Title: Functional electrical stimulation of shoulder muscles for supporting the limb against gravity. Authors: Ariel B. Thomas ${ }^{1,2}$, Rawan AlGhawi, Y.J. Chang, Sergiy Yakovenko, and Valeriya Gritsenko ${ }^{1,2}$

\section{Affiliations:}

${ }^{1}$ Department of Human Performance, Division of Physical Therapy, School of Medicine West Virginia University, Morgantown, WV, 26506

${ }^{2}$ Rockefeller Neuroscience Institute, Department of Neuroscience, West Virginia University, Morgantown, WV, 26506

${ }^{3}$ WVU Center for Teleneurology and Telestroke, Morgantown, WV, 26506

${ }^{4}$ Department of Neurology, School of Medicine, West Virginia University, Morgantown, WV, 26506

Corresponding author: Valeriya Gritsenko, PO Box 9226, One Medical Center Drive, Morgantown, WV, 26505-9226. Phone: (304) 293-7719

Running Head: dynamic assessment of post-stroke reaching 


\section{Introduction}

A common result of a stroke is weakened muscles of the arm, including those that support the shoulder joint. Weakened shoulder muscles often lead to partial dislocation of the head of the humerus from the glenohumeral joint due to the force of gravity, clinically referred to as shoulder subluxation. This causes further complications that limit the recovery of motor function. This includes limitations in hand function, which can impair the ability to perform everyday tasks. Shoulder pain associated with subluxation typically occurs within 6 months after stroke onset. Shoulder subluxation is correlated with hemorrhagic type of stroke and loss of proprioceptive sense, and negatively correlated with Brunnstrom's stage of arm recovery (Auchstaetter et al. 2016). Researchers suggest an overall incidence of $17-81 \%$ of shoulder pain along with shoulder subluxation in stroke patients, leading to impaired achievement of full functional capacity (Faghri et al. 1994), Such outcomes have a negative effect on the overall prognosis and thus impede their rehabilitation. Consequences are not limited to the restriction of movement: shoulder subluxation can also cause further injury to neurovascular tissues around the shoulder joint.

Shoulder subluxation is commonly treated with the therapeutic application of cyclic NMES and FES stimulation to the Medial Deltoid, Posterior Deltoid and/or Supraspinatus, with significant results seen both preventatively and therapeutically (Arya et al. 2018; Church et al. 2006; Faghri et al. 1994; Kobayashi et al. 1999; Koyuncu et al. 2010; Linn et al. 1999; Mangold et al. 2009; Wang et al. 2000, 2002). In clinical settings, electrical stimulation can be used for improving muscle strength, increasing range of motion, reducing edema, decreasing atrophy, healing tissue, and decreasing pain (Doucet et al. 2012). Shoulder subluxation is also treated with gravitational support, most often a sling or brace (Paci et al. 2005). While a sling significantly restricts movement, which is important in post-stroke recovery, modern shoulder support braces are flexible and do not impair movement. A FES assistive device offers an alternative solution by directly activating muscles that are responsible for gravity support, and has potential to be incorporated into a supportive brace to accelerate recovery (Sun et al. 2018). The method applied here and in the previous chapter has the benefit of being similar to therapeutic application of FES in stroke patients to prevent and treat shoulder subluxation, a form of shoulder dislocation caused by weakened shoulder muscles.

Shoulder subluxation is caused due to the effect of gravity pulling on the arm, dislocating the humerus from the glenoid fossa and increasing the glenohumeral space. The addition of gravity support at the shoulder can prevent this, and we believe that using FES to provide this support 
can have additional benefits, such as strengthening of the muscles that support this joint. We hypothesize that the stimulation will have an effect of reducing the glenohumeral space when the arm is resting and a movement is induced passively, reflecting that it directly causes an effect related to relieving shoulder subluxation. Glenohumeral distance and glenohumeral height changes due to stimulation will be recorded using high-resolution ultrasound of the shoulder, and measured over the course of a 1-1.5 hour session in a group of healthy, young controls. We additionally had two stroke subjects that performed the reaching FES task from Aim 2 return to analyze this effect in a small case study involving 30 minutes of stimulation for the anterior deltoid followed by 30 minutes stimulation of the medial deltoid. We hypothesize that the effect on the glenohumeral joint due to FES will be similar in these groups, and will remain effective throughout the length of the application.

We hope to develop a wearable FES assistive strategy that provides gravity support at the shoulder and can be used for the length of a typical physical therapy session or longer. More development is necessary to examine the effects of stimulation over prolonged use. Biomechanically, muscle fatigue is described as the inability to maintain the expected musclegenerated force output over time (Porter and Whelan 2009). Muscle fatigue can measured experimentally using electromyography (EMG) to examine fatigue dynamics at the level of each individual muscle, or by using kinematic and force sensors to measure muscle-generated output from muscle groups. FES is more fatiguing than natural movement in healthy individuals, due to differences in muscle fiber recruitment (Bigland-Ritchie et al. 1979) along with lower selectivity of which muscles will be activated (Kuhn et al. 2010). Over a period of use, FES will produce less muscle-generated force output than it initially provided (Downey et al. 2017). Therefore, we wanted to quantify this fatigue generated when using FES on muscles of the shoulder. Research has shown that after stroke, muscles fatigue faster than in healthy individuals (Hu et al. 2006; Riley and Bilodeau 2002). Because of this, we expect stroke participants to fatigue faster with FES than healthy controls.

Approach

Our aim was to test the impact of shoulder FES on the glenohumeral joint. Shoulder subluxation is a subtype of shoulder dislocation where the head of the humerus only partially dislocates, causing a palpable gap between the acromion and the humeral head. Therefore, studies using FES as a treatment tool for shoulder subluxation would benefit from the use of ultrasound to visualize changes in muscular activity affecting the size of this gap, but few have applied this 
strategy. As far as we can report, no studies have used ultrasound concurrently with shoulder FES to understand the relationship to shoulder subluxation. High-resolution ultrasonography has been highly implemented in diagnosing shoulder pathology that conventional radiographs were not able to demonstrate (Vitoonpong and Chang 2021). Due to its low cost, advances in technology, and reduced radiation exposure, ultrasound is superior to magnetic resonance or computed tomography in diagnosing shoulder pathology. It is also the first-line imaging instrument in diagnosing shoulder pathologies. Multiple studies showed it has high sensitivity and specificity of confirming shoulder dislocation and relocation (Suethanapornkul et al. 2008). Ultrasound should be used before and after reduction to confirm successful relocation of the joint. Knowledge of anatomy and high experience of sonographers are vital factors for detecting any pathology. In this study, FES was applied with certain parameters capable of producing shoulder muscle contractions (anterior deltoid and medial deltoid) via intact peripheral nerves. We used ultrasound to visualize the shoulder architecture when stimulated by FES and when not stimulated. We tested the right shoulder muscles of control participants with no existing health conditions impairing their full range of movements, along with the left impaired shoulder of two stroke participants (reported in chapter 3). 


\section{Methods}

\section{Subjects}

We recruited 13 control participants (6 female/7 male, ages 24+/-3.2) with no neuropathological motor conditions to provide a baseline of non-impaired movement for this FES task. Control subjects were excluded if they had any neurological conditions that could affect their motor control. We recruited 2 stroke survivors with right hemisphere strokes and a different hemiparetic symptoms. These subjects were also recruited for chapter 3 and will therefore be referred to as S2 and S3 to remain consistent. Stroke participant 2 (S2) had moderate impairment with overactive flexor synergies, Stroke participant 3 (S3) Severe impairment (NIH Stroke Scale = 6) with flaccid hemiparesis. Exclusion criteria included the inability to produce visible movement with their shoulder and elbow or the inability to provide written consent to participate. All participants were right hand dominant. The study and the consent procedure were approved by the Institutional Review Board of West Virginia University (Protocol \# 19065977769). Subjects provided written consent before participation.

Table 1. Participants

\begin{tabular}{|c|c|c|c|c|c|}
\hline Participants & $\begin{array}{l}\text { Weight } \\
\text { (kg) }\end{array}$ & $\begin{array}{l}\text { Height } \\
\text { (cm) }\end{array}$ & Sex & $\begin{array}{l}\text { Age } \\
\text { (years) }\end{array}$ & Muscle Tested \\
\hline \multicolumn{6}{|l|}{ Control } \\
\hline C1 & 69 & 164 & $\mathrm{~m}$ & 22 & MD \\
\hline $\mathrm{C2}$ & 70 & 150 & $f$ & 18 & MD \\
\hline C3 & 66 & 170 & $f$ & 27 & $A D$ \\
\hline C4 & 74.8 & 170 & $f$ & 19 & $A D$ \\
\hline C5 & 56.6 & 158.8 & $f$ & 24 & $A D$ \\
\hline C6 & 50 & 160 & $f$ & 30 & MD \\
\hline C7 & 71 & 171 & $\mathrm{~m}$ & 23 & $A D$ \\
\hline $\mathrm{C8}$ & 90 & 185 & $\mathrm{~m}$ & 24 & $A D$ \\
\hline $\mathrm{C9}$ & 70 & 170 & $\mathrm{~m}$ & 27 & MD \\
\hline c10 & 55 & 170 & $\mathrm{~m}$ & 24 & $A D$ \\
\hline c11 & 80 & 169 & $\mathrm{~m}$ & 22 & $A D$ \\
\hline c12 & 100 & 168 & $\mathrm{~m}$ & 25 & MD \\
\hline
\end{tabular}




\begin{tabular}{|c|c|c|c|c|c|}
\hline c13 & 81 & 170 & f & 23 & MD \\
\hline Participant & $\begin{array}{l}\text { Weight } \\
(\mathrm{kg})\end{array}$ & $\begin{array}{l}\text { Height } \\
(\mathrm{cm})\end{array}$ & Sex & $\begin{array}{l}\text { Age } \\
\text { (yea }\end{array}$ & Muscle Tested \\
\hline \multicolumn{6}{|l|}{ Stroke } \\
\hline S2 & 95 & 177 & $\mathrm{~m}$ & 59 & MD\&AD \\
\hline S2 Details: & \multicolumn{5}{|c|}{ Right Lacunar Stroke, 12 Years Post-Stroke, NIH SS = 3} \\
\hline S3 & 100 & 192 & $\mathrm{~m}$ & 49 & MD\&AD \\
\hline S3 Details & \multicolumn{5}{|c|}{$\begin{array}{l}\text { Right MCA Stroke, } 8 \text { Years Post-Stroke, NIH SS }=6 \text {, history of post-stroke } \\
\text { subluxation }\end{array}$} \\
\hline
\end{tabular}

Task

Control participants (Table 1) were stimulated on either the MD $(n=10)$ or $A D(n=11)$ for 1.5 hours. Stroke participants (Table 1, also reported in Table 1 of Chapter 3 ) were stimulated first on the $A D$ and then the MD for 30 minutes each. Stimulation was applied to the anterior deltoid or medial deltoid at amplitude levels adjusted to produce an initial 30 degrees of arm movement. This amplitude remained constant through the remainder of the experiment for controls, but was modified in stroke participants.

Each stimulation cycle took 6 seconds (1s ramp up, 2 s on, 1 sec ramp down, 2 sec off). The change in flexion or abduction angle was calculated using traditional video recordings by 2 blinded raters in the first 2 subjects. In video recordings, the change in angle was measured every 2 minutes from the maximum to the minimum shoulder angle position during a stimulation cycle. For the remainder of subjects, Notch motion capture was used to collect angle measurements at a rate of $40 \mathrm{hz}$ for flexion and abduction angles. We estimated the change in shoulder angle amplitude for each stimulation pulse over time using a 9 second maximum and minimum moving window function with a step size of 6 seconds. For consistency with camera-based measurements, we averaged this angle difference for every one minute interval throughout the experiment. We used ultrasound to visualize a FES-induced change in the glenohumeral space every 10 minutes for controls and 5 minutes for stroke subjects. Each recording captured one full cycle of stimulation. Two blinded raters measured the change in both the overall distance between the glenoid fossa and the head of the humerus (glenhumDist) and the change in the vertical distance between these points (glenhumHeight) to examine the effect of medial or anterior deltoid 
contraction (Lahham et al. 2016). During the session of FES, stroke subjects were assessed for fatigue three times: pre-session, midway through the session, and post-session, using a modified hand-held dynamometer.

\section{Ultrasound of the glenohumeral (GH) joint}
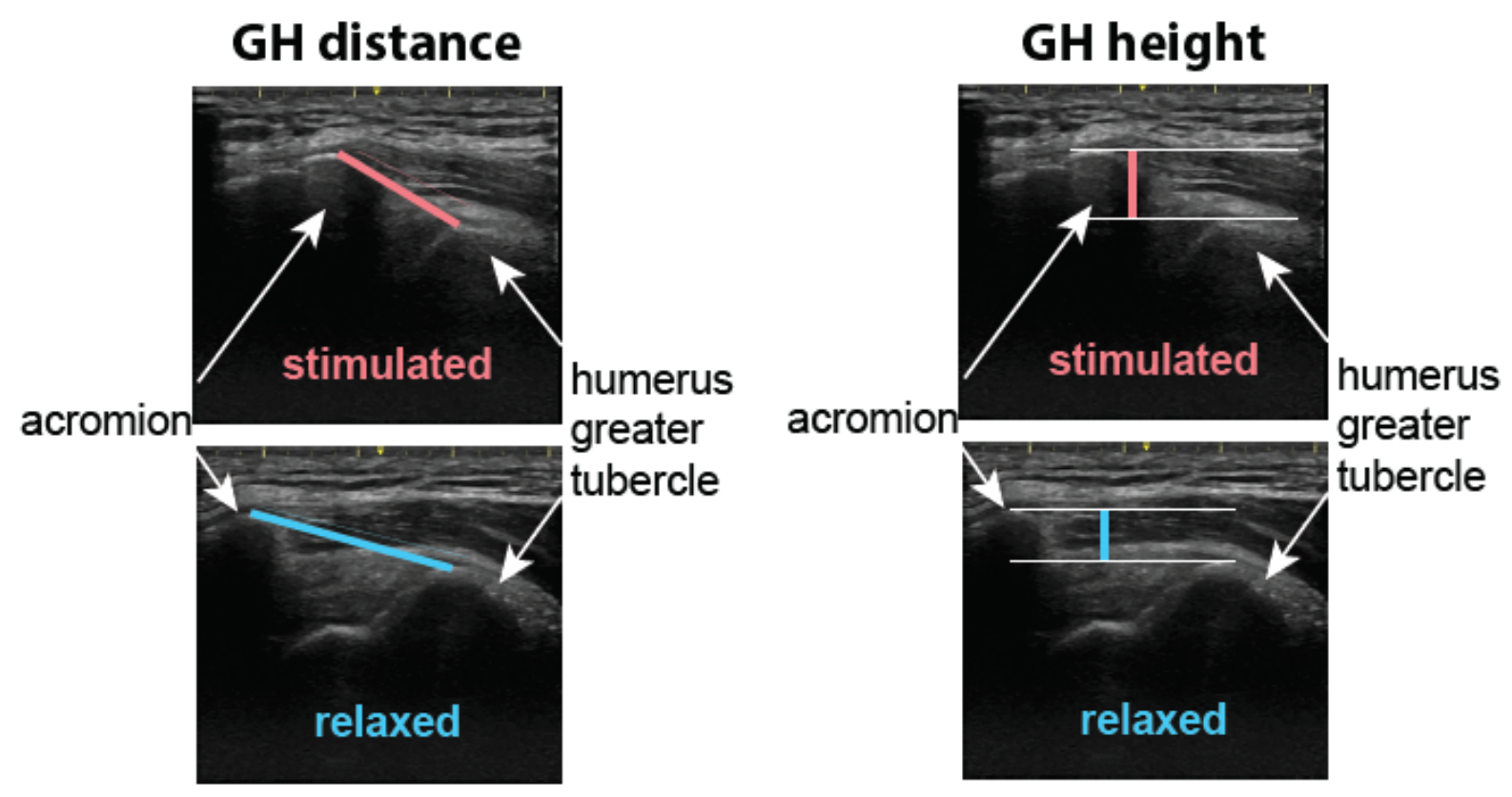

Figure 1. Shoulder ultrasound measurements. The images show position of two bony landmarks during tetanic stimulation of supraspinatus (top) and without stimulation (relaxed, bottom). Colored lines illustrate how measurements of $\mathrm{GH}$ distance and $\mathrm{GH}$ height were obtained. Figure and legend adapted from AlGhawi et al., 2021.

Results

Angles elicited by stimulation in controls and stroke subjects was similar. The average AD flexion angle for controls over the course of the experiment was $15.8 \pm 5$ degrees, compared to the stroke participant's average flexion angle of 14.9 (S2) and 16.5 (S3) degrees (Fig. 2A). The average MD abduction angle for controls over the course of the experiment was $10.6 \pm 6$ degrees, compared to the stroke participant's average flexion angle of 8.7 (S2) and 14.7 (S3) degrees (Fig. 2B). 
Unexpectedly, we did not see a consistent decrease in the induced shoulder angle over time in controls over their 1.5 hour stimulation session. In anterior deltoid stimulation, there was surprisingly an increase in average FES induced shoulder in $5 / 6$ subjects The regression coefficient ( $r$ ) for subjects C4, C5, C7, C8, C10, and C11 was -0.0084, 0.1136, 0.0406, 0.0054, 0.0366 , and 0.3227 . The change in flexion angle over time was significant for 3 subjects (C5: R2 $=0.6090, p<0.001, c 7: R 2=0.0816, p=0.01, C 11: R 2=0.6606, p<0.001)$. These 3 subjects had an increase in shoulder flexion angle over time, while no subjects had a significant decrease in shoulder angle.

For the medial deltoid, 4/6 subjects with a decrease in FES induced abduction angle, although the other two subjects had these angles increase over time. The regression coefficient $(r)$ for subjects C2, C3, C6, C9, C12, and C13 was 0.0195, -0.0959, -0.1021, -0.1133, -0.0591, and 0.0674 . The change in abduction angle over time was significant for three subjects, two of which had a significant decrease in shoulder abduction angle over time $(C 6: R 2=0.1219, p=0.0162$, C9: $R 2=0.1697, p<0.001)$, while one subject had an increase in abduction angle $(C 13: R 2=$ $0.2460, p<0.001)$. This suggests either that there is minimal fatigue generated by these parameters of FES in the course of a 1-1.5 hour session.

A

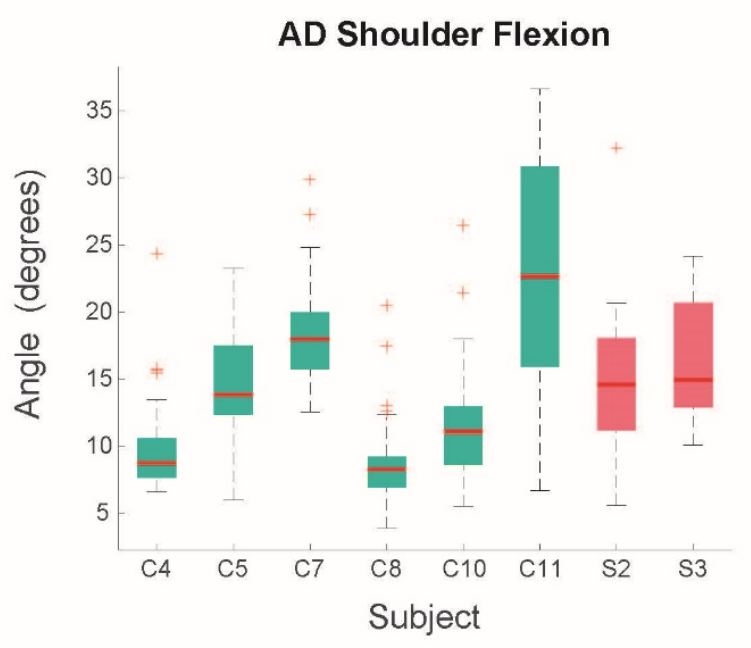

B

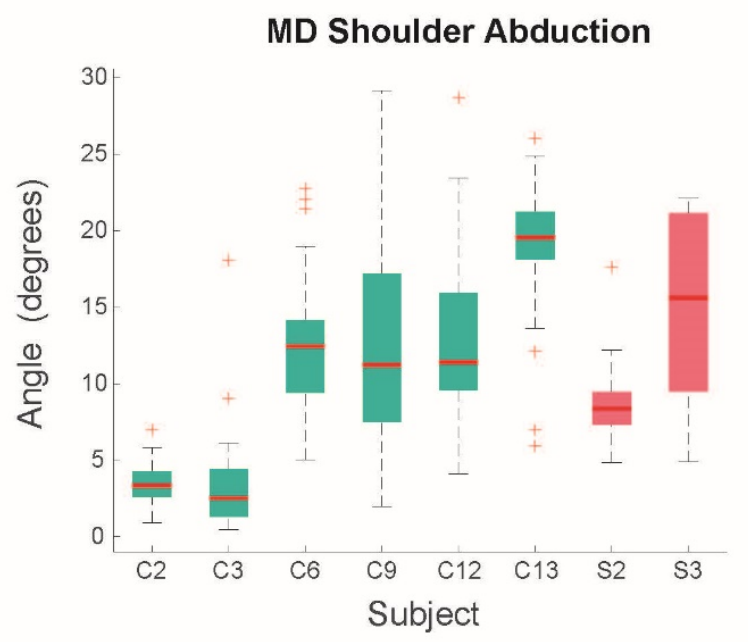

Figure 2. Changes in shoulder angle for healthy controls and stroke subjects. $\boldsymbol{A}$. Change in shoulder flexion due to anterior deltoid stimulation (,B. Change in shoulder abduction angle due to medial deltoid stimulation (bottom). For subjects C2 and C3, angles were measured from video every 2 minutes by blinded raters. For all other subjects, angles were calculated using Notch motion capture, with the average change in angle for every pulse cycle averaged every 1 minute. (circles). 
Stroke Subjects 2 and 3 from the FES study described in chapter 3 were brought back to examine the effects of fatigue due to stimulation. Due to the expected accelerated levels of fatigue compared to controls, the amplitude of stimulation was increased by the experimenter to retain a fixed movement amplitude over 30 minutes of stimulation at rest. This occurred as expected in participant S2 (Fig. 3). For stroke participant 2, shoulder stimulation amplitude was increased from $22 \mathrm{~mA}$ to $29 \mathrm{~mA}$ for the $\mathrm{AD}$ and from $43 \mathrm{~mA}$ to $48 \mathrm{~mA}$ for the MD. This was successful in keeping shoulder flexion and abduction angles fixed (regression coefficient: $A D=-0.0055, M D=$ -0.0040).. We used the change in set amplitude over time to calculate the FES induced shoulder angle per $\mathrm{mA}$ of stimulation. This did not change significantly over the course of the experiment. In stroke participant S3, the change in shoulder angle increased over time for both the anterior deltoid (0.2191) and medial deltoid $r=0.4279)$, although it was only a significant increase in the medial deltoid $(R 2=0.4395, p=0.0014)$. This illustrates that the increase in stimulation amplitude was larger than necessary to maintain a fixed amplitude. Shoulder FES amplitude was increased from $31 \mathrm{~mA}$ to $38 \mathrm{~mA}$ for the $\mathrm{AD}$ and from $32 \mathrm{~mA}$ to $38 \mathrm{~mA}$ for the $\mathrm{MD}$, and the induced change in flexion and abduction increased over time. The FES induced shoulder angle per $m A$ of stimulation did not increase for the anterior deltoid and the medial deltoid over time (Fig 3).

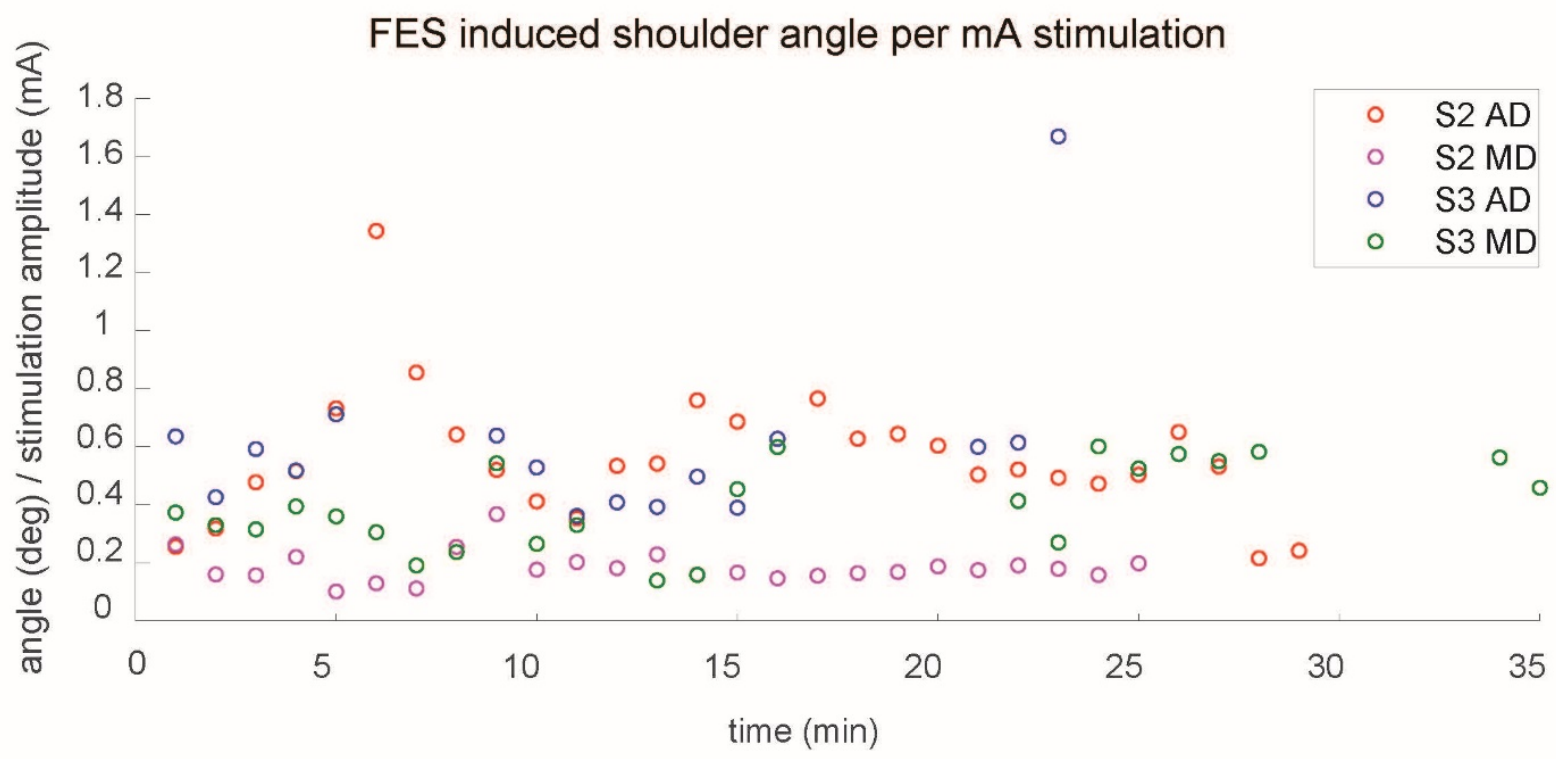

Figure 3. FES induced change in shoulder angle per mA of stimulation for two stroke participants. Shoulder stimulation was manually increased over each 30 minute session in stroke subjects at increments of 5 minutes, if necessary, to retain the same amount of movement. Change in height or distance per $\mathrm{mV}$ 
stimulation was calculated per timepoint of averaged stimulation amplitude for stroke subject S2 (AD in red, $M D$ in magenta) and stroke subject $S 3$ ( $A D$ in blue, MD in green), and plotted over time

The change in glenohumeral height was small ( AD: $-0.4883+/-0.331 \mathrm{~mm}$, MD: $-0.2330+/-0.4424$ $\mathrm{mm}$ ) and unchanging over time in controls (linear regression $r$ between -0.1 and $0.1, p>0.05$ for all controls), while the change in the glenohumeral distance was large ( AD: $-0.4883+/-0.331 \mathrm{~mm}$, MD: ) and unchanging. 4 out of $6 \mathrm{MD}$ control subjects showed an insignificant increasing change in $\mathrm{GH}$ distance caused by electrical stimulation to the medial deltoid over time (regression coefficient (r): $\mathrm{C} 4=0.0189, \mathrm{C} 5=-0.0145, \mathrm{C} 7=-0.0142, \mathrm{C} 8=-0.0303, \mathrm{C} 10=0.0205$, and $\mathrm{C} 11$ $=-0.0125)$, and 4 out of $6 A D$ control subjects showed an insignificant increased change in $\mathrm{GH}$ height caused by electrical stimulation to the medial deltoid over time (regression coefficient $(r)$ : $\mathrm{C} 2=0.0189, \mathrm{C} 3=-0.0145, \mathrm{C} 6=-0.0142, \mathrm{C} 9=-0.0303, \mathrm{C} 12=0.0205$, and $\mathrm{C} 13=-0.0125)$. We were unable to tell if the FES induced effect on the glenohumeral gap changed over time, with high variability between data points. Overall, we see that the stimulation had an effect of decreasing the glenohumeral distance in controls. 
A

Glenohumeral Distance
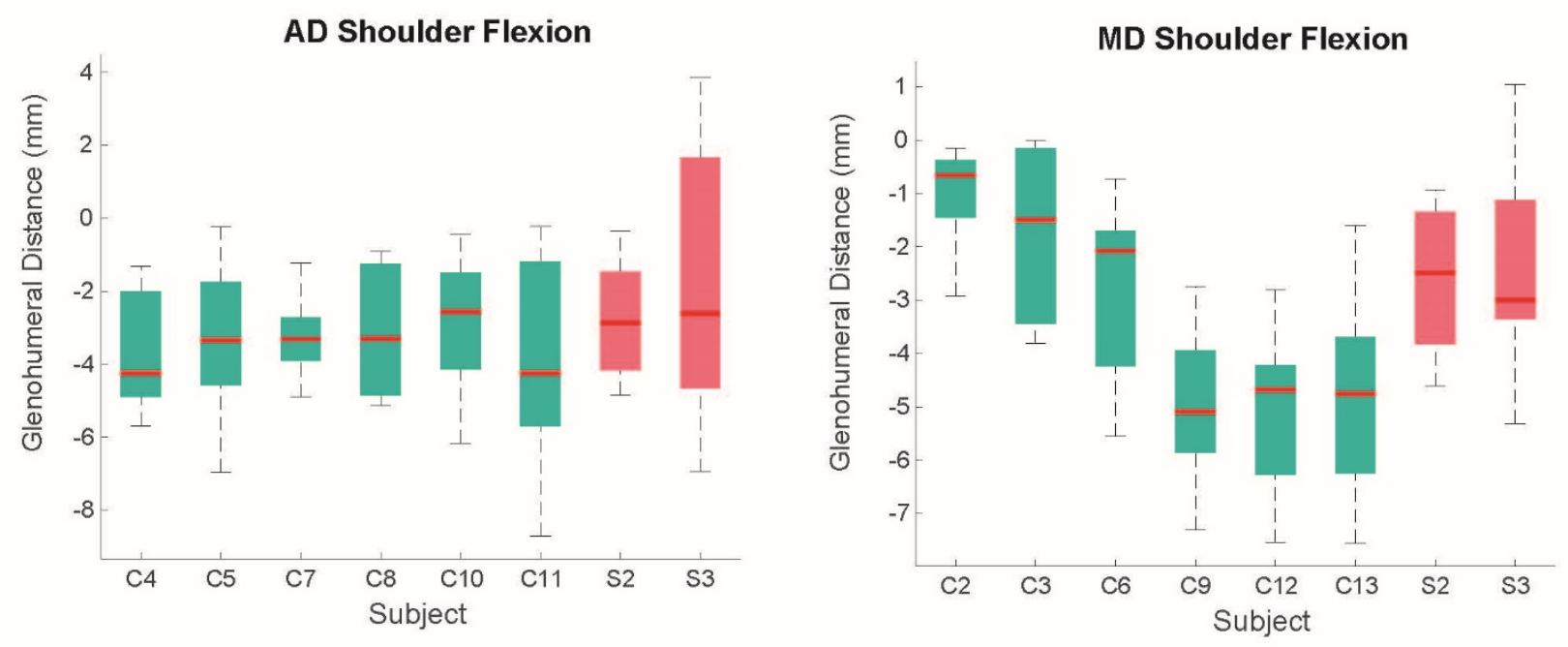

B

Glenohumeral Height
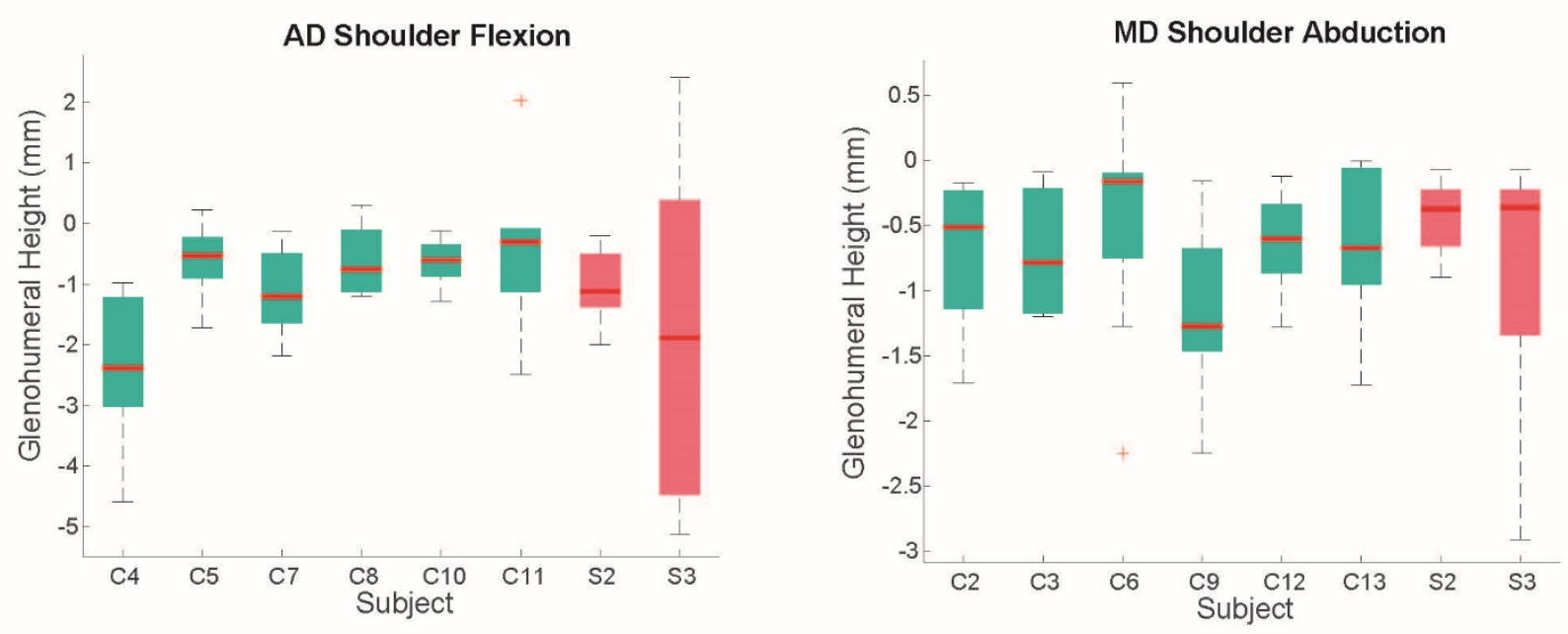

Figure 4. Changes in glenohumeral distance and height with FES in healthy controls. A. Change in glenohumeral distance $(\mathrm{mm})$ induced by stimulation to the Anterior Deltoid ( $A D$, left) and the Medial Deltoid (MD, right). Controls are plotted in green and stroke subjects are plotted in red. A negative value reflects a decrease in glenohumeral distance, with more negative values reflecting a greater decrease. $\boldsymbol{B}$. Change in glenohumeral height induced by stimulation to the Anterior Deltoid (AD, left) and the Medial Deltoid (MD, right). Controls are plotted in green and stroke subjects are plotted in red. A negative value reflects a decrease in glenohumeral distance, with more negative values reflecting a greater decrease. Measurements were taken by blinded raters using high-resolution ultrasound of the shoulder. 
In stroke participant S2, the glenohumeral distance and height decreased similarly to controls (AD: $\mathrm{GHd}=-2.7612, \mathrm{GHh}=-0.2541, \mathrm{MD}: \mathrm{GHd}=-2.6143, \mathrm{GHh}=-0.1300$, Fig. 4). The change in glenohumeral distance over time was insignificant ( $p>0.05$ ), at a rate of $-0.1 \mathrm{~mm} / \mathrm{min}$ for $A D$ stimulation and $-0.08 \mathrm{~mm} / \mathrm{min}$ for $\mathrm{MD}$ stimulation, and a change in glenohumeral height at a rate of $-0.03 \mathrm{~mm} / \mathrm{min}$ for $\mathrm{AD}$ stimulation and $-0.01 \mathrm{~mm} / \mathrm{min}$ for MD stimulation. In stroke participant S3, the change in glenohumeral distance and height were greater than that of controls (AD: GHd = 1.9501, $\mathrm{GHh}=-0.6798, \mathrm{MD}: \mathrm{GHd}=-2.4580, \mathrm{GHh}=-0.2638$, Fig. 4). This subject has a history of shoulder subluxation, and this was visible in these measurements. The change in glenohumeral height over time also decreased more rapidly than controls, with a change in glenohumeral height at a rate of $-0.13 \mathrm{~mm} / \mathrm{min}$ for $A D$ stimulation and $-0.1 \mathrm{~mm} / \mathrm{min}$ for MD stimulation. However, while there was a small decrease in glenohumeral distance for AD was not significant and in MD it slightly increased over time, likely related to the increase in stimulation induced shoulder angle, with at a rate of $-0.1 \mathrm{~mm} / \mathrm{min}$ for $A D$ stimulation and $0.08 \mathrm{~mm} / \mathrm{min}$ for $\mathrm{MD}$ stimulation. Overall regressions were not fit well $(p>0.05)$, due to high variability and low sample size.

Stroke participants S2 showed decreasing maximal shoulder flexion force as a result of stimulation of the anterior deltoid, which was regained after stimulation of the medial deltoid (Table 2). The effect of stimulation on this subject's abduction, adduction, and internal rotation maximal force decreased by about $10 \mathrm{~kg}$ after anterior deltoid stimulation and remained at that strength after medal deltoid stimulation. Shoulder extension and external rotation had no change in this subject. For participant S3, anterior and medial deltoid stimulation had no decrease in strength for any recorded degree of freedom of the shoulder. Unexpectedly, recorded strength increased for abduction after anterior deltoid stimulation in this subject.

Table 2. Force measurements for stroke participants. Force measurements (kg) were taken using a handheld dynamometer pre stimulation, mid stimulation (post-AD and pre-MD), and post stimulation. Three measurements for each value shown were taken and averaged. Shoulder degrees of freedom examined include flexion (Flex), extension (Ext), abduction (Abd), adduction (Add), internal rotation (InRot), and external rotation (ExRot).

\begin{tabular}{|c|c|c|c|c|c|c|c|}
\hline \multirow{3}{*}{$\begin{array}{l}\text { Subject } \\
\text { S2 }\end{array}$} & \multirow{3}{*}{$\begin{array}{l}\text { Test } \\
\text { pre }\end{array}$} & \multicolumn{6}{|c|}{ Force (kg) } \\
\hline & & Flex & \multirow[t]{2}{*}{ Ext } & Abd & Add & InRot & ExRot \\
\hline & & 10 & & 23 & 25 & 24 & 5 \\
\hline & mid & 6 & 9 & 12 & 15 & 12 & 2 \\
\hline & post & 13 & 9 & 13 & 14 & 12 & 2 \\
\hline \multirow[t]{3}{*}{ S3 } & pre & 12 & 8 & 7 & 11 & 5 & 1 \\
\hline & mid & 11 & 10 & 10 & 25 & 7 & 1 \\
\hline & post & 15 & 8 & 9 & 18 & 8 & 2 \\
\hline
\end{tabular}




\section{Discussion}

We examined the effect of shoulder FES application used to move the arm into flexion or abduction. We found that in a resting arm of healthy controls, extended stimulation of 1-1.5 hours for the deltoid moved the shoulder into flexion or abduction and reduced the distance between the scapular and humerus within the glenohumeral joint. In two stroke subjects, the amplitude of stimulation was sometimes increased to maintain the same amplitude of joint angle change over a 30 minute stimulation session of the AD followed by a 30 minute stimulation session on the MD. This increase was less than $10 \mathrm{~mA}$, and angles remained constant or increased over time with some decrease in shoulder strength.

Overall, there were low levels of fatigue observed over the length of a typical therapeutic session The action of FES to partially support the weight of the arm against gravity remained effective in this time frame. The noticeable effect on the glenohumeral distance did not diminish over the length of this session. Therefore, there may be an additional benefit of using FES to strengthen shoulder muscles weakened by stroke to maintain a constant glenohumeral gap. This suggests that FES may be effective in a combined treatment aimed at both reducing shoulder subluxation and facilitating arm function in people with stroke. FES applied to both the anterior and medial deltoid had comparable effects on the glenohumeral gap, and these muscles are both viable targets for FES-based therapy. The glenohumeral height was not effected in healthy controls by stimulation, suggesting that it is not a useful measure of the effect of deltoid FES on glenohumeral joint. However, it is not clear if this would apply to individuals with larger than normal glenohumeral height differences due to shoulder subluxation. This case series is limited by the small number of subjects, but it benefits from examining the same stroke participants that completed the FES assisted reaching task. In this way, we have a greater understanding of how the assistive stimulation both improves reach and grasp, has no significant fatigue, and impacts the glenohumeral joint in a way that may be beneficial in preventing shoulder subluxation.

FES has been used to produce movement for people who have lost partial or complete function of their muscles. It also is used therapeutically to strengthen shoulder muscles to treat and prevent a common and debilitating post-stroke injury, shoulder subluxation. Wearable FES technology can make it possible for a patient to have easy access to stimulation necessary to assist with movement and prevent injury. 


\section{Chapter 5: Conclusions and Future Directions.}

\section{Summary}

In this thesis, I applied neuromechanical concepts to test new methods of assessment and restoration of motor function after stroke. In chapter 1 , I discussed how joint coupling emerges after stroke, its neuromechanical causes, and how it can be treated using gravitational support. In chapter 2 I hypothesized that quantifying post-stroke motor deficits using active muscle torque is a superior metric compared to joint angles. I applied an inverse dynamic simulation technique to calculate gravity dependent and gravity independent muscle torques from motion capture. This was performed in a young, healthy aged, and stroke population using a multi-joint unrestrained 3-dimensional reaching task. I found that the dynamic component of muscle torque closely resembles angular velocity but is better at distinguishing stroke-related motor deficits. Using the gravity-dependent muscle torques from this chapter, I was able to see what the profile of gravity-resisting muscle torque for the anterior and medial deltoid would be for a forward and lateral reach, respectively.

In chapter 3 I hypothesized that gravitational support in the form of functional electrical stimulation to muscles of the shoulder improves grasping. I designed and tested a game integrated with movement-triggered FES and presented in virtual reality. In this system, the individual was instructed to reach to and grasp targets as they appeared, and the system provide FES to the shoulder to assist with movement. This FES assistance was given in the same profile as the gravity-resisting muscle torques calculated in chapter 2 , thereby providing assistance against gravity for the shoulder during the reach. In the two stroke patients that performed this task, we saw improvements in hand muscle coordination, grasping aperture, movement speed. Reach trajectory was unaffected. Two other, more impaired stroke subjects were recruited that were not able to open their hands in order to trigger the task in virtual reality. In these subjects we applied cyclic stimulation with the same parameters and asked to reach to and attempt to grasp physical objects and found similar improvements in hand muscle activity. This implies that even off-the-shelf neuromuscular electrical stimulation units can be used in this type of application and even with subjects that are more impaired.

Finally, in chapter 4 I hypothesized that FES of deltoid muscles can reduce the glenohumeral distance in the shoulder joint. I recorded the effect of extended use of FES and examined 
changes in movement. Based on previous studies that show decreases in induced torque over periods of constant cyclic stimulation (Downey et al. 2017), we expected that shoulder FES caused gradual decline in the abduction or flexion angle over time. We quantified this change in detail over a 1.5 hour stimulation session in controls. We further observed that the stimulation of deltoid muscle reduced the glenohumeral distance in all participants. This suggests that stimulation to the anterior and medial deltoid in patterns to assist with movement may be used to support the shoulder integrity in presence of reduced muscle activity after stroke. This suggests that FES may be effective in preventing or reducing some secondary effects of stroke, such as shoulder separation or subluxation. Altogether, the outcomes of these studies offer strong evidence for the usefulness of neuromechanical concepts for the development of new methods of assessment and treatment of motor impairment after stroke.

\section{Limitations}

Our studies were performed on a small sample size of stroke participants. This reduces the application of our results to the stroke population. The data that we did acquire was highdimensional, consisting of recordings from many muscles, and detailed multi-joint kinematic recordings for many tasks. However, there is always the risk of finding significance due to the sheer volume of data recorded in these studies. To minimize the number of tests in chapter 2 , we analyzed the data by comparing differences across multiple dimentions of data, as seen in the 112-dimensional clustering algorithm and corrected for familywise error. In chapter 3 , we examined only the kinematics and muscles of the hand and corrected for familywise error. Chapter 4 had a repeated-measures experimental design, with subjects serving as their own controls. However, we performed only two case studies with stroke participants, and each control subgroup had only 6 subjects. Additionally, our glenohumeral analysis would have benefited from higher frequency of ultrasound recordings, which would have allowed us to have a better understanding of the change in glenohumeral measurements over time.

Chapter 3 would greatly benefit from the incorporation of predictive control or reactive control algorithms. Currently, it is limited in its use as an assistive device because it cannot predict movement. In our specific VR application, FES was simply triggered by interaction events in VR, such as when subjects approached the virtual target. With modifications, we could use motion capture or a wearable sensor to detect the shoulder angle, which would then drive closed-loop control of the assistive stimulation. Additionally, if this is to be an assistive application, then it 
should be integrated in a wearable way that is user-friendly and designed to minimize fatigue over time.

\section{Future Directions}

Advancements in Control Mechanisms for FES

Currently, transcutaneous FES can be operated through manual control, movement of the contralateral limb, and through residual muscle activity in the impaired limb. When the state of the disease prevents the use of these approaches, brain computer interfaces $(\mathrm{BCl})$ are emerging as an alternative. Ultimately, the most significant change in FES over the next decade will be changes in control methods. To advance to at-home or assistive applications, control of FES devices will be handed over from the physical therapist to the patient. This raises additional safety concerns, as the stimulation would be unmonitored, and patients may use the device in ways that are not specifically instructed. Transcutaneous FES is not dangerous when used correctly and is currently sold over the counter. However, individuals still may not remove or turn off the device when it is appropriate to do so, leading to the potential for skin burns and electrical shock. This can be minimized through adding limitations to the stimulation parameters that cannot be modified by the subject, along with providing them training and technology assistance. Regardless, the potential benefit of restoring independent movement control to a stroke patient is an imperative for translational medicine.

The integration of voluntary motor control into FES involves using residual muscle activity to support the user's movement attempt. This can be done through sensors of movement or EMG recordings. One strategy, used by Klauer 2016 involves amplifying volitionally initiated shoulder abductions (monitored through kinematic sensors) and then providing FES to enhance and stabilize movement. Hybrid Assistive Neuromuscular Dynamic Stimulation (HANDS) employs ipsilateral control of reaching and grasping by combining closed-loop EMG controlled transcutaneous FES with a non-robotic wrist-hand splint for support (Fujiwara et al. 2017). This last design has made it out of the lab and into the clinic, where it is currently in clinical use for hemiplegic patients.

Many studies have used contralateral limb control to drive an FES for rehabilitation, as it is an effective way to discern proper muscle activation patterns for an intended movement. Often, this is performed using a glove, such as that of the Bionic Glove(Micera et al. 2010) . Some researchers wanted to explore further the significance of using electrical stimulation enables a 
functionally significant movement versus simply electrically activating the muscles. A study of 80 chronic stroke patients (greater than 6 months post-stroke) and found that contralaterally controlled FES for hand opening was more effective at improving hand dexterity than cyclic neuromuscular electrical stimulation (cNMES) for hand opening. However, the significance between the two electrical stimulation strategies was only found in participants less than 2 years post stroke with moderate hand impairment at baseline, as this group had the largest overall motor recovery after treatment. This experiment was notable in that the rehabilitation sessions for both FES and cNMES could be performed at home without the direct supervision of a specialist.

Cortical control of FES is also emerging as a possible neuroprosthetic technique. The coordination of reaching and grasping movements of a paralyzed arm was performed in a paraplegic patent using cortical control of implanted FES (Ajiboye et al. 2017). Two intracortical microelectrode arrays were implanted in the hand area of his motor cortex and 36 percutaneous electrodes were implanted in his right arm to stimulate hand, elbow, and shoulder muscles. The tasks used a motorized support brace for gravitational assistance and to provide humeral abduction and adduction under cortical control. The patient could volitionally reach to drink a mug of coffee and feed himself. This provides evidence that cortically controlled FES is a clinically viable neuroprostheses for restoration of reaching and grasping.

A recent feasibility study developed an EEG-based brain computer interface $(\mathrm{BCI})$ and achieved real-time control of an upper-limb FES in healthy subjects (Vidaurre et al. 2016). EEG measures voltage fluctuations within the neurons of the brain, and has been used effectively to control $\mathrm{BCl}$ devices. In their test of linear control accuracy, the participants were instructed to 'point' toward a target. They were able to achieve a mean selection accuracy of $82.5 \%$. However, this selection task was performed using software that added a friction factor that would slow the target icon whenever the patient was pointing within the threshold of a target and should be repeated looking at the kinematic features of the limb rather than a selection task. Nevertheless, this experiment provides the first evidence that a non-invasive $\mathrm{BCl}$ could be used to control a non-invasive FES-neuroprosthesis. The results of these efforts make progress toward the control of an impaired limb with the sole use of brain activity. As exciting as this progress is for the future of neuroprosthetics, dynamic changes in the brain after stroke would make designing a controller that depends on its activity a difficult task.

Cost-Effective sensors for Wearable Technologies 
Incorporating FES electrode arrays into wearable technology provides a means for the instruments to become more user-friendly and would allow patients to administer the therapy athome. However, this still requires significant steps to be made, such as optimizing electrode current parameters for specific muscle activation and integrating a control strategy that can correct for misalignment, while still reducing the cost of the equipment. This is not to say that take-home FES therapeutic devices have not been successful. Most notably, a study of 80 chronic stroke patients (greater than 6 months post-stroke) found that contralaterally controlled FES for hand opening was more effective at improving hand dexterity than cyclic neuromuscular electrical stimulation (cNMES) for hand opening (Knutson et al. 2016). This experiment was notable in that the rehabilitation sessions for both FES and cNMES were performed at home without the direct supervision of a specialist.

Surface electrode arrays, first used to activate forearm muscles (Nathan 1979), provide less fatiguing and more selective muscle activation than single electrode applications. Placing set of small electrodes in a fixed position before application provided a significant simplification to electrode placement. In addition, the ability to independently control each electrode in the array gives the ability to optimize the activation of specific motor units, thereby improving the ability to generate a specific target movement.

In research settings over the last 20 years, FES for rehabilitation has repeatedly shown excellent performance. However, only a limited number of FES devices are being used by clinicians. Cyclic NMES is a similar technique that electrically activates muscles rhythmically but does not incorporate neuroprosthetics and is not used for functional control of an intentional movement in the upper limbs. Cyclic NMES is being rapidly incorporated into physical therapy across the country. FES is shown to have a greater rehabilitative effect than cyclic FES in upper limbs in research settings and has the ability to restore function immediately for daily tasks, but it is a less attractive option for clinicians because of three major factors: 1) High cost of instruments, 2) Lack of user-friendliness, and 3) Poor performance in the clinic due to misalignment of instruments. Incorporating FES electrode arrays into wearable technology provides a means for the instruments to become more user-friendly and would allow patient to administer the therapy at-home. However, this still requires significant development, such as optimizing electrode arrays for specific muscle activation and developing and integrating control algorithms that can correct for misalignment, while still reducing the cost of the equipment. 
A flexible, breathable, and lightweight device for wearable FES that can be manufactured at low cost through screen printing of polymer based pastes on fabric (Yang et al. 2018). The fabric electrode array (FEA) has demonstrated successful operation has been demonstrated to achieve clinical standards of pointing, pinching and hand opening, and the materials with skin contact been cytotoxicity and are biocompatible. The clinical feasibility for this method now rests on designing fast and effective closed-loop feedback control strategies that make the operation of the device easy enough to perform at home.

Model-based feedback control

The first control strategies for FES involved sequentially stimulating with single-pad electrodes to locate the best location for muscle activation, which is time-consuming and has accuracy limited by clinician skill. This is seen through manually repositioning a single electrode (Nathan 1979) or testing each array element individually. The Popovic lab recently provided a strategy to select array electrodes without manually testing each electrode, by recording joint angle data with kinematic sensors during individual electrode stimulations and allowing the system to minimizing a cost function to tell which electrodes are positioned usefully for a given task (Malešević et al. 2012). In 2016, the same lab developed a method to select appropriate positions of the stimulating electrodes using EMG activity maps estimated from electrode arrays and wearable amplifiers on the nonparetic and paretic forearms (Popović Maneski et al. 2016). After identifying sites on the paretic arm that had a decrease or delay in EMG activity compared to that location on the nonparetic arm during an attempt at the same hand opening and grasping task, they stimulated the paretic sites using transcutaneous FES electrode arrays. They also included in their FES application the co-activation of flexors and extensors to stabilize the wrist joint. Electrode feedback controllers have also been developed that incorporate joint angle for disturbance compensation, while simplifying other aspects of the control design procedure to make it suitable for application in a clinical setting (Freeman et al. 2016). Force/torque sensors, kinematic sensors, accelerometers, and low-cost flex sensors each have the potential to be used to design feedback controls that improve the operation of an FES device.

Use of co-activation in antagonistic muscles to stabilize control of upper limbs

The Schauer lab developed a control strategy for the amplification of volitional shoulder abductions through FES and showed an effective prevention of fatigue as well as arm weight support (Klauer et al. 2016). FES-induced muscle recruitment in this study was generated 
based on the abduction angle of the arm. Feedback control was provided through their designed recruitment controller, which included a user-optimized filtering algorithm of the FES-evoked EMG to provide the amount of FES stimulation that promotes effective movement without fatiguing the muscle. They then applied antagonist muscle co-contraction to the anterior and posterior deltoid to support the arm, and found that this increased movement stability, but also accelerated muscle fatigue. Another group performed an antagonist co-activation strategy for the wrist, but found that incorporating increased joint-stiffness for wrist extension and flexion led to increased stability, but also increased errors in movement accuracy for various functional tasks (Bó et al. 2016).

Combination of FES with rehabilitation robotics

Combinatorial therapies, such as hybrid FES/robotic systems, have shown impressive functional recovery results and may be the future for rehabilitation. An early study in 2005 designed and tested a hybrid robotic FES assisted exercise therapy system (NESS Handmaster) for improvement of hand opening in people with moderate to severe hemiparesis due to stroke(Ring and Rosenthal 2005). A FES stimulator and forearm-mounted robotic device were used to assist with hand opening and closing, and had statistically significant improvement in hand opening for weeks after therapy compared to traditional therapy. A 2016 study showed that subthreshold FES can increase the effectiveness of shoulder and elbow robotic training in patients with stroke hemiparesis (Miyasaka et al. 2016). It is important to note that most studies of FES used mobilized braces for stability during movement. The HANDS treatment, as discussed earlier, used a splint for stability of the hand. It follows that incorporating a robotic assistance could replace the gravity support device and providing additional rehabilitative assistance. However, cost may become a major factor in this application.

\section{$\underline{\text { Conclusions }}$}

I have shown that neuromechanical concepts can be used to develop and test new methods of assessment and treatment of motor impairment after stroke. Developing this technology further into autonomous wearables will provide the means for the patients to administer their therapy at-home. Over the last few years, optimized feedback control systems and low-cost and lightweight electrode array systems have been designed that will drastically enhance the practicality of FES-based assistive technology for home-based stroke rehabilitation. There are several different modalities that could be chosen by the patient to control the FES 
neuroprosthesis, depending on the extent of the motor impairment, or simply their preference. In addition, augmenting a support brace or robotic device with FES may further enhance rehabilitation of motor impairment. Ultimately, it will be interesting to see how these independent improvements on the FES technique will come together to enhance the field. 


\section{$\underline{\text { References }}$}

Achour S Ben, Pascual O. Glia: The many ways to modulate synaptic plasticity. Neurochemistry International 57: 440-445, 2010.

Ajiboye AB, Willett FR, Young DR, Memberg WD, Murphy BA, Miller JP, Walter BL, Sweet JA, Hoyen HA, Keith MW, Peckham PH, Simeral JD, Donoghue JP, Hochberg LR, Kirsch RF. Restoration of reaching and grasping movements through brain-controlled muscle stimulation in a person with tetraplegia: a proof-of-concept demonstration. Lancet 389: 18211830, 2017.

Akbarian S, Grüsser OJ, Guldin WO. Corticofugal connections between the cerebral cortex and brainstem vestibular nuclei in the macaque monkey. J Comp Neurol 339: 421-437, 1994.

Alt Murphy M, Murphy S, Persson HC, Bergström U-B, Sunnerhagen KS. Kinematic Analysis Using 3D Motion Capture of Drinking Task in People With and Without Upperextremity Impairments. J Vis Exp , 2018. doi:10.3791/57228.

Amano Y, Noma T, Etoh S, Miyata R, Kawamura K, Shimodozono M. Reaching exercise for chronic paretic upper extremity after stroke using a novel rehabilitation robot with arm-weight support and concomitant electrical stimulation and vibration: before-and-after feasibility trial. Biomed Eng Online 19: 28, 2020.

Amaral AI, Meisingset TW, Kotter MR, Sonnewald U. Metabolic Aspects of NeuronOligodendrocyte-Astrocyte Interactions. Frontiers in Endocrinology 4: 54, 2013.

Arthur D, Vassilvitskii S. k-means++: The Advantages of Careful Seeding. In: Proceedings of the Eighteenth Annual ACM-SIAM Symposium on Discrete Algorithms,. SODA 2007. New Orleans, Louisiana: 2007, p. 11.

Arya KN, Pandian S, Puri V. Rehabilitation methods for reducing shoulder subluxation in poststroke hemiparesis: a systematic review. Top Stroke Rehabil 25: 68-81, 2018.

Auchstaetter N, Luc J, Lukye S, Lynd K, Schemenauer S, Whittaker M, Musselman KE. Physical Therapists' Use of Functional Electrical Stimulation for Clients With Stroke: Frequency, Barriers, and Facilitators. Phys Ther 96: 995-1005, 2016.

Bagesteiro LB, Sainburg RL. Handedness: Dominant Arm Advantages in Control of Limb Dynamics. Journal of Neurophysiology 88: 2408-2421, 2002.

Baker SN. The primate reticulospinal tract, hand function and functional recovery. Journal of Physiology 589: 5603-5612, 2011.

Baker SN, Zaaimi B, Fisher KM, Edgley SA, Soteropoulos DS. Pathways mediating functional recovery. Prog Brain Res 218: 389-412, 2015.

Bastian AJ, Martin TA, Keating JG, Thach WT. Cerebellar ataxia: abnormal control of interaction torques across multiple joints. Journal of Neurophysiology 76: 492-509, 1996. 
Beer RF, Dewald JPA, Dawson ML, Rymer WZ. Target-dependent differences between free and constrained arm movements in chronic hemiparesis. Exp Brain Res 156: 458-470, 2004.

Beer RF, Dewald JPA, Rymer WZ. Deficits in the coordination of multijoint arm movements in patients with hemiparesis: evidence for disturbed control of limb dynamics. Experimental Brain Research 131: 305-319, 2000.

Beer RF, Given JD, Dewald JP. Task-dependent weakness at the elbow in patients with hemiparesis. Arch Phys Med Rehabil 80: 766-772, 1999.

Benjamin EJ, Muntner P, Alonso A, Bittencourt MS, Callaway CW, Carson AP, Chamberlain AM, Chang AR, Cheng S, Das SR, Delling FN, Djousse L, Elkind MSV, Ferguson JF, Fornage M, Jordan LC, Khan SS, Kissela BM, Knutson KL, Kwan TW, Lackland DT, Lewis TT, Lichtman JH, Longenecker CT, Loop MS, Lutsey PL, Martin SS, Matsushita K, Moran AE, Mussolino ME, O'Flaherty M, Pandey A, Perak AM, Rosamond WD, Roth GA, Sampson UKA, Satou GM, Schroeder EB, Shah SH, Spartano NL, Stokes A, Tirschwell DL, Tsao CW, Turakhia MP, VanWagner LB, Wilkins JT, Wong SS, Virani SS, On behalf of the American Heart Association Council on Epidemiology and Prevention Statistics Committee and Stroke Statistics Subcommittee. Heart Disease and Stroke Statistics-2019 Update: A Report From the American Heart Association. Circulation 139, 2019.

Bhadane MY, Gao F, Francisco GE, Zhou P, Li S. Correlation of Resting Elbow Angle with Spasticity in Chronic Stroke Survivors. Front Neurol 6: 183, 2015.

Bigland-Ritchie B, Jones DA, Woods JJ. Excitation frequency and muscle fatigue: electrical responses during human voluntary and stimulated contractions. Exp Neurol 64: 414-427, 1979.

Bó APL, da Fonseca LO, de Sousa ACC. FES-induced co-activation of antagonist muscles for upper limb control and disturbance rejection. Med Eng Phys 38: 1176-1184, 2016.

Bourbonnais D, Vanden Noven S, Carey KM, Rymer WZ. Abnormal spatial patterns of elbow muscle activation in hemiparetic human subjects. Brain 112 ( Pt 1): 85-102, 1989.

Bowden MG, Clark DJ, Kautz SA. Evaluation of abnormal synergy patterns poststroke: relationship of the Fugl-Meyer Assessment to hemiparetic locomotion. Neurorehabil Neural Repair 24: 328-337, 2010.

Brodal A. Self-observations and neuro-anatomical considerations after a stroke. Brain 96: 675694, 1973.

Brown P. Pathophysiology of spasticity. J Neurol Neurosurg Psychiatry 57: 773-777, 1994.

Brunnstrom S. Motor testing procedures in hemiplegia: based on sequential recovery stages. Phys Ther 46: 357-375, 1966.

Burke D, Wissel J, Donnan GA. Pathophysiology of spasticity in stroke. Neurology 80: S2026, 2013. 
Calautti C, Naccarato M, Jones PS, Sharma N, Day DD, Carpenter AT, Bullmore ET, Warburton EA, Baron J-C. The relationship between motor deficit and hemisphere activation balance after stroke: A 3T fMRI study. Neuroimage 34: 322-331, 2007.

Cannell J, Jovic E, Rathjen A, Lane K, Tyson AM, Callisaya ML, Smith ST, Ahuja KD, Bird M-L. The efficacy of interactive, motion capture-based rehabilitation on functional outcomes in an inpatient stroke population: a randomized controlled trial. Clin Rehabil 32: 191$200,2018$.

Carp JS, Wolpaw JR. Motor Neurons and Spinal Control of Movement. In: eLS. American Cancer Society.

Chang Y-J, Chen S-F, Huang J-D. A Kinect-based system for physical rehabilitation: A pilot study for young adults with motor disabilities. Research in Developmental Disabilities 32: 25662570, 2011.

Chen Y-T, Li S, DiTommaso C, Zhou P, Li S. Possible Contributions of Ipsilateral Pathways From the Contralesional Motor Cortex to the Voluntary Contraction of the Spastic Elbow Flexors in Stroke Survivors: A TMS Study. Am J Phys Med Rehabil 98: 558-565, 2019.

Chou C-H, Wang T, Sun X, Niu CM, Hao M, Xie Q, Lan N. Automated functional electrical stimulation training system for upper-limb function recovery in poststroke patients. Medical Engineering \& Physics 84: 174-183, 2020.

Choudhury S, Singh R, Shobhana A, Sen D, Anand SS, Shubham S, Gangopadhyay S, Baker MR, Kumar H, Baker SN. A Novel Wearable Device for Motor Recovery of Hand Function in Chronic Stroke Survivors. Neurorehabil Neural Repair 34: 600-608, 2020.

Church C, Price C, Pandyan AD, Huntley S, Curless R, Rodgers H. Randomized controlled trial to evaluate the effect of surface neuromuscular electrical stimulation to the shoulder after acute stroke. Stroke 37: 2995-3001, 2006.

Cirstea MC, Mitnitski AB, Feldman AG, Levin MF. Interjoint coordination dynamics during reaching in stroke. Experimental Brain Research 151: 289-300, 2003.

Clark RA, Pua Y-H, Bryant AL, Hunt MA. Validity of the Microsoft Kinect for providing lateral trunk lean feedback during gait retraining. Gait \& Posture 38: 1064-1066, 2013.

Coderre AM, Amr Abou Zeid, Dukelow SP, Demmer MJ, Moore KD, Demers MJ, Bretzke H, Herter TM, Glasgow JI, Norman KE, Bagg SD, Scott SH. Assessment of Upper-Limb Sensorimotor Function of Subacute Stroke Patients Using Visually Guided Reaching. Neurorehabil Neural Repair 24: 528-541, 2010.

Davidson AG, Buford JA. Motor outputs from the primate reticular formation to shoulder muscles as revealed by stimulus-triggered averaging. $J$ Neurophysiol 92: 83-95, 2004.

Davidson AG, Buford JA. Bilateral actions of the reticulospinal tract on arm and shoulder muscles in the monkey: stimulus triggered averaging. Exp Brain Res 173: 25-39, 2006. 
Davidson AG, Schieber MH, Buford JA. Bilateral spike-triggered average effects in arm and shoulder muscles from the monkey pontomedullary reticular formation. J Neurosci 27: 80538058, 2007.

Debicki DB, Gribble PL. Persistence of inter-joint coupling during single-joint elbow flexions after shoulder fixation. Exp Brain Res 163: 252-257, 2005.

Denny-Brown DE, Gilman S, Van der Meulen J. Patterns of cortical ablations leading to dystonic postures. Trans Am Neurol Assoc 89: 117-121, 1964.

Dewald JP, Pope PS, Given JD, Buchanan TS, Rymer WZ. Abnormal muscle coactivation patterns during isometric torque generation at the elbow and shoulder in hemiparetic subjects. Brain 118 ( Pt 2): 495-510, 1995.

Dewald JP, Sheshadri V, Dawson ML, Beer RF. Upper-limb discoordination in hemiparetic stroke: implications for neurorehabilitation. Top Stroke Rehabil 8: 1-12, 2001.

Di Pino G, Pellegrino G, Assenza G, Capone F, Ferreri F, Formica D, Ranieri F, Tombini M, Ziemann U, Rothwell JC, Di Lazzaro V. Modulation of brain plasticity in stroke: a novel model for neurorehabilitation. Nat Rev Neurol 10: 597-608, 2014.

Dimyan MA, Cohen LG. Neuroplasticity in the context of motor rehabilitation after stroke. Nat Rev Neurol 7: 76-85, 2011.

Doucet BM, Lam A, Griffin L. Neuromuscular electrical stimulation for skeletal muscle function. Yale J Biol Med 85: 201-215, 2012.

Dounskaia N, Wang W. A preferred pattern of joint coordination during arm movements with redundant degrees of freedom. Journal of Neurophysiology 112: 1040-1053, 2014.

Downey RJ, Merad M, Gonzalez EJ, Dixon WE. The Time-Varying Nature of Electromechanical Delay and Muscle Control Effectiveness in Response to Stimulation-Induced Fatigue. IEEE Transactions on Neural Systems and Rehabilitation Engineering 25: 1397-1408, 2017.

Drew T, Prentice S, Schepens B. Cortical and brainstem control of locomotion. Progress in Brain Research 143: 251-261, 2004.

Duncan P, Studenski S, Richards L, Gollub S, Lai SM, Reker D, Perera S, Yates J, Koch V, Rigler S, Johnson D. Randomized clinical trial of therapeutic exercise in subacute stroke. Stroke 34: 2173-2180, 2003a.

Duncan P, Studenski S, Richards L, Gollub S, Lai SM, Reker D, Perera S, Yates J, Koch V, Rigler S, Johnson D. Randomized Clinical Trial of Therapeutic Exercise in Subacute Stroke. Stroke 34: 2173-2180, 2003 b.

Dutta C, Hadley EC. The Significance of Sarcopenia in Old Age. The Journals of Gerontology Series A: Biological Sciences and Medical Sciences 50A: 1-4, 1995. 
Edström E, Altun M, Bergman E, Johnson H, Kullberg S, Ramírez-León V, Ulfhake B. Factors contributing to neuromuscular impairment and sarcopenia during aging. Physiology \& Behavior 92: 129-135, 2007.

Ellis MD, Carmona C, Drogos J, Dewald JPA. Progressive Abduction Loading Therapy with Horizontal-Plane Viscous Resistance Targeting Weakness and Flexion Synergy to Treat Upper Limb Function in Chronic Hemiparetic Stroke: A Randomized Clinical Trial. Front Neurol 9: $71,2018$.

Ellis MD, Holubar BG, Acosta AM, Beer RF, Dewald JPA. Modifiability of abnormal isometric elbow and shoulder joint torque coupling after stroke. Muscle Nerve 32: 170-178, 2005.

Ellis MD, Kottink AIR, Prange GB, Rietman JS, Buurke JH, Dewald JPA. Quantifying loss of independent joint control in acute stroke with a robotic evaluation of reaching workspace. Annu Int Conf IEEE Eng Med Biol Soc 2011: 8231-8234, 2011.

Ellis MD, Schut I, Dewald JPA. Flexion synergy overshadows flexor spasticity during reaching in chronic moderate to severe hemiparetic stroke. Clin Neurophysiol 128: 1308-1314, 2017.

Ellis MD, Sukal T, DeMott T, Dewald JPA. Augmenting clinical evaluation of hemiparetic arm movement with a laboratory-based quantitative measurement of kinematics as a function of limb loading. Neurorehabil Neural Repair 22: 321-329, 2008.

Ellis MD, Sukal-Moulton TM, Dewald JPA. Impairment-Based 3-D Robotic Intervention Improves Upper Extremity Work Area in Chronic Stroke: Targeting Abnormal Joint Torque Coupling With Progressive Shoulder Abduction Loading. IEEE Trans Robot 25: 549-555, 2009.

Evans WJ. What Is Sarcopenia? The Journals of Gerontology Series A: Biological Sciences and Medical Sciences 50A: 5-8, 1995.

Evans WJ, Campbell WW. Sarcopenia and Age-Related Changes in Body Composition and Functional Capacity. The Journal of Nutrition 123: 465-468, 1993.

Faghri PD, Rodgers MM, Glaser RM, Bors JG, Ho C, Akuthota P. The effects of functional electrical stimulation on shoulder subluxation, arm function recovery, and shoulder pain in hemiplegic stroke patients. Arch Phys Med Rehabil 75: 73-79, 1994.

Fellows SJ, Kaus C, Ross HF, Thilmann AF. Agonist and antagonist EMG activation during isometric torque development at the elbow in spastic hemiparesis. Electroencephalogr Clin Neurophysiol 93: 106-112, 1994a.

Fellows SJ, Kaus C, Thilmann AF. Voluntary movement at the elbow in spastic hemiparesis. Ann Neurol 36: 397-407, 1994 b.

Fitts PM. The information capacity of the human motor system in controlling the amplitude of movement. Journal of Experimental Psychology 47: 381-391, 1954. 
Freeman CT, Yang K, Tudor J, Kutlu M. Feedback control of electrical stimulation electrode arrays. Med Eng Phys 38: 1185-1194, 2016.

Fugl-Meyer AR, Jääskö L, Leyman I, Olsson S, Steglind S. The post-stroke hemiplegic patient. 1. a method for evaluation of physical performance. Scandinavian Journal of Rehabilitation Medicine 7: 13-31, 1974.

Fugl-Meyer AR, Jääskö L, Leyman I, Olsson S, Steglind S. The post-stroke hemiplegic patient. 1. a method for evaluation of physical performance. Scand J Rehabil Med 7: 13-31, 1975.

Fujiwara T, Kawakami M, Honaga K, Tochikura M, Abe K. Hybrid Assistive Neuromuscular Dynamic Stimulation Therapy: A New Strategy for Improving Upper Extremity Function in Patients with Hemiparesis following Stroke. Neural Plast 2017: 2350137, 2017.

Gentili R, Cahouet V, Papaxanthis C. Motor planning of arm movements is directiondependent in the gravity field. Neuroscience 145: 20-32, 2007.

Gottlieb GL, Song Q, Almeida GL, Hong D-A, Corcos D. Directional Control of Planar Human Arm Movement. Journal of Neurophysiology 78: 2985-2998, 1997.

Gowland C, Stratford P, Ward M, Moreland J, Torresin W, Van Hullenaar S, Sanford J, Barreca S, Vanspall B, Plews N. Measuring physical impairment and disability with the Chedoke-McMaster Stroke Assessment. Stroke 24: 58-63, 1993.

Gribble PL, Ostry DJ. Compensation for Interaction Torques During Single- and Multijoint Limb Movement. Journal of Neurophysiology 82: 2310-2326, 1999.

Hara Y, Ogawa S, Tsujiuchi K, Muraoka Y. A home-based rehabilitation program for the hemiplegic upper extremity by power-assisted functional electrical stimulation. Disabil Rehabil 30: 296-304, 2008.

Hebb DO. The organization of behavior; a neuropsychological theory. Oxford, England: Wiley, 1949.

Herbert WJ, Davidson AG, Buford JA. Measuring the motor output of the pontomedullary reticular formation in the monkey: do stimulus-triggered averaging and stimulus trains produce comparable results in the upper limbs? Exp Brain Res 203: 271-283, 2010.

Herbert WJ, Powell K, Buford JA. Evidence for a role of the reticulospinal system in recovery of skilled reaching after cortical stroke: initial results from a model of ischemic cortical injury. Exp Brain Res 233: 3231-3251, 2015.

Hsieh Y, Wu C, Lin K, Chang Y, Chen C, Liu J. Responsiveness and Validity of Three Outcome Measures of Motor Function After Stroke Rehabilitation. Stroke 40: 1386-1391, 2009.

Hu XL, Tong KY, Hung LK. Firing properties of motor units during fatigue in subjects after stroke. Journal of Electromyography and Kinesiology 16: 469-476, 2006. 
Hughes AM, Freeman CT, Burridge JH, Chappell PH, Lewin PL, Rogers E. Shoulder and elbow muscle activity during fully supported trajectory tracking in people who have had a stroke. J Electromyogr Kinesiol 20: 465-476, 2010.

Jang SH, Chang CH, Lee J, Kim CS, Seo JP, Yeo SS. Functional role of the corticoreticular pathway in chronic stroke patients. Stroke 44: 1099-1104, 2013.

Kahn LE, Lum PS, Rymer WZ, Reinkensmeyer DJ. Robot-assisted movement training for the stroke-impaired arm: Does it matter what the robot does? Journal of rehabilitation research and development 43: 619-630, 2014.

Kamper DG, Fischer HC, Cruz EG, Rymer WZ. Weakness is the primary contributor to finger impairment in chronic stroke. Arch Phys Med Rehabil 87: 1262-1269, 2006.

Keeling AB, Piitz M, Semrau JA, Hill MD, Scott SH, Dukelow SP. Robot enhanced stroke therapy optimizes rehabilitation (RESTORE): a pilot study. J Neuroeng Rehabil 18: 10, 2021.

Keizer K, Kuypers HG. Distribution of corticospinal neurons with collaterals to the lower brain stem reticular formation in monkey (Macaca fascicularis). Exp Brain Res 74: 311-318, 1989.

Kerr AL, Cheng S-Y, Jones TA. Experience-dependent neural plasticity in the adult damaged brain. J Commun Disord 44: 538-548, 2011.

Ketcham CJ, Dounskaia NV, Stelmach GE. Multijoint movement control: the importance of interactive torques. In: Progress in Brain Research. Elsevier, p. 207-218.

Ketcham CJ, Seidler RD, Van Gemmert AWA, Stelmach GE. Age-Related Kinematic Differences as Influenced by Task Difficulty, Target Size, and Movement Amplitude. The Journals of Gerontology: Series B 57: P54-P64, 2002.

Kim YH, You SH, Kwon YH, Hallett M, Kim JH, Jang SH. Longitudinal fMRI study for locomotor recovery in patients with stroke. Neurology 67: 330-333, 2006.

Klauer C, Ferrante S, Ambrosini E, Shiri U, Dähne F, Schmehl I, Pedrocchi A, Schauer T. A patient-controlled functional electrical stimulation system for arm weight relief. Med Eng Phys 38: 1232-1243, 2016.

Kline TL, Schmit BD, Kamper DG. Exaggerated interlimb neural coupling following stroke. Brain 130: 159-169, 2007.

Knutson JS, Gunzler DD, Wilson RD, Chae J. Contralaterally Controlled Functional Electrical Stimulation Improves Hand Dexterity in Chronic Hemiparesis: A Randomized Trial. Stroke 47: 2596-2602, 2016.

Knutson JS, Wilson RD, Makowski NS, Chae J. Chapter 94 - Functional Electrical Stimulation for Return of Function After Stroke. In: Neuromodulation (Second Edition), edited by Krames ES, Peckham PH, Rezai AR. Academic Press, p. 1137-1145. 
Kobayashi H, Onishi H, Ihashi K, Yagi R, Handa Y. Reduction in subluxation and improved muscle function of the hemiplegic shoulder joint after therapeutic electrical stimulation. $J$ Electromyogr Kinesiol 9: 327-336, 1999.

Koutsou AD, Moreno JC, Del Ama AJ, Rocon E, Pons JL. Advances in selective activation of muscles for non-invasive motor neuroprostheses. J Neuroeng Rehabil 13: 56, 2016.

Koyuncu E, Nakipoğlu-Yüzer GF, Doğan A, Ozgirgin N. The effectiveness of functional electrical stimulation for the treatment of shoulder subluxation and shoulder pain in hemiplegic patients: A randomized controlled trial. Disabil Rehabil 32: 560-566, 2010.

Krakauer JW, Carmichael ST. Broken Movement: The Neurobiology of Motor Recovery After Stroke. MIT Press, 2017a.

Krakauer JW, Carmichael ST. Broken Movement: The Neurobiology of Motor Recovery After Stroke. MIT Press, $2017 \mathrm{~b}$.

Kuhn A, Keller T, Lawrence M, Morari M. The influence of electrode size on selectivity and comfort in transcutaneous electrical stimulation of the forearm. IEEE Trans Neural Syst Rehabil Eng 18: 255-262, 2010.

Kuypers HG. THE DESCENDING PATHWAYS TO THE SPINAL CORD, THEIR ANATOMY AND FUNCTION. Prog Brain Res 11: 178-202, 1964.

Kwakkel G, van Wegen EEH, Burridge JH, Winstein CJ, van Dokkum LEH, Alt Murphy M, Levin MF, Krakauer JW, ADVISORY group. Standardized Measurement of Quality of Upper Limb Movement After Stroke: Consensus-Based Core Recommendations From the Second Stroke Recovery and Rehabilitation Roundtable. Neurorehabil Neural Repair 33: 951958, 2019.

Lan Y, Yao J, Dewald JPA. The Impact of Shoulder Abduction Loading on Volitional Hand Opening and Grasping in Chronic Hemiparetic Stroke. Neurorehabil Neural Repair 31: 521$529,2017$.

Lawrence DG, Kuypers HG. The functional organization of the motor system in the monkey. II. The effects of lesions of the descending brain-stem pathways. Brain 91: 15-36, 1968.

\section{Lawrence ES, Coshall C, Dundas R, Stewart J, Rudd AG, Howard R, Wolfe CDA.} Estimates of the prevalence of acute stroke impairments and disability in a multiethnic population. Stroke 32: 1279-1284, 2001.

Le Seac'h AB, McIntyre J. Multimodal reference frame for the planning of vertical arms movements. Neuroscience Letters 423: 211-215, 2007.

van der Lee J, Beckerman $\mathbf{H}$, Lankhorst $\mathbf{G}$, Bouter $\mathbf{L}$. The responsiveness of the Action Research Arm and the Fugl-Meyer Assessment Scale in Chronic Storke Patients. Journal of Rehabilitation Medicine 33: 110-113, 2001a. 
van der Lee J, Beckerman $\mathbf{H}$, Lankhorst $\mathbf{G}$, Bouter $\mathbf{L}$. The responsiveness of the Action Research Arm and the Fugl-Meyer Assessment Scale in Chronic Storke Patients. Journal of Rehabilitation Medicine 33: 110-113, $2001 \mathrm{~b}$.

Levin MF. Interjoint coordination during pointing movements is disrupted in spastic hemiparesis. Brain 119: 281-293, 1996.

Levin MF, Kleim JA, Wolf SL. What do motor "recovery" and "compensation" mean in patients following stroke? Neurorehabil Neural Repair 23: 313-319, 2009.

Levin MF, Liebermann DG, Parmet Y, Berman S. Compensatory Versus Noncompensatory Shoulder Movements Used for Reaching in Stroke. Neurorehabil Neural Repair 30: 635-646, 2016.

Li S. Spasticity, Motor Recovery, and Neural Plasticity after Stroke. Front Neurol 8: 120, 2017.

Li S, Bhadane M, Gao F, Zhou P. The Reticulospinal Pathway Does Not Increase Its Contribution to the Strength of Contralesional Muscles in Stroke Survivors as Compared to Ipsilesional Side or Healthy Controls. Front Neurol 8: 627, 2017.

Li S, Chen Y-T, Francisco GE, Zhou P, Rymer WZ. A Unifying Pathophysiological Account for Post-stroke Spasticity and Disordered Motor Control. Front Neurol 10: 468, 2019.

Li S, Francisco GE. New insights into the pathophysiology of post-stroke spasticity. Front Hum Neurosci 9: 192, 2015.

Linn SL, Granat MH, Lees KR. Prevention of shoulder subluxation after stroke with electrical stimulation. Stroke 30: 963-968, 1999.

Lum P, Reinkensmeyer D, Mahoney R, Rymer WZ, Burgar C. Robotic Devices for Movement Therapy After Stroke: Current Status and Challenges to Clinical Acceptance. Topics in Stroke Rehabilitation 8: 40-53, 2002.

Malešević NM, Popović Maneski LZ, Ilić V, Jorgovanović N, Bijelić G, Keller T, Popović DB. A multi-pad electrode based functional electrical stimulation system for restoration of grasp. J Neuroeng Rehabil 9: 66, 2012.

Mangold S, Schuster C, Keller T, Zimmermann-Schlatter A, Ettlin T. Motor training of upper extremity with functional electrical stimulation in early stroke rehabilitation. Neurorehabil Neural Repair 23: 184-190, 2009.

Manto M-U, Bosse P. Directional tuning of speed-related activation for reaching in the vertical plane in cerebellar ataxia. Neurological Research 25: 434-444, 2003.

Matsuyama K, Takakusaki K, Nakajima K, Mori S. Multi-segmental innervation of single pontine reticulospinal axons in the cervico-thoracic region of the cat: anterograde PHA-L tracing study. J Comp Neurol 377: 234-250, 1997. 
McCall AA, Miller DM, Yates BJ. Descending Influences on Vestibulospinal and Vestibulosympathetic Reflexes. Front Neurol 8: 112, 2017.

McPherson JG, Stienen AH, Drogos JM, Dewald JP. Modification of Spastic Stretch Reflexes at the Elbow by Flexion Synergy Expression in Individuals With Chronic Hemiparetic Stroke. Arch Phys Med Rehabil 99: 491-500, 2018.

Micera S, Keller T, Lawrence M, Morari M, Popović DB. Wearable neural prostheses. Restoration of sensory-motor function by transcutaneous electrical stimulation. IEEE Eng Med Biol Mag 29: 64-69, 2010.

Miller LC, Dewald JPA. Involuntary paretic wrist/finger flexion forces and EMG increase with shoulder abduction load in individuals with chronic stroke. Clin Neurophysiol 123: 1216-1225, 2012.

Miyasaka H, Orand A, Ohnishi H, Tanino G, Takeda K, Sonoda S. Ability of electrical stimulation therapy to improve the effectiveness of robotic training for paretic upper limbs in patients with stroke. Med Eng Phys 38: 1172-1175, 2016.

Morice AH, Bennett RT, Chaudhry MA, Cowen ME, Griffin SC, Loubani M. Effect of bitter tastants on human bronchi. Nat Med 17: 775, 2011.

Mousavi Hondori H, Khademi M. A Review on Technical and Clinical Impact of Microsoft Kinect on Physical Therapy and Rehabilitation. Journal of Medical Engineering 2014: 1-16, 2014.

Murphy MA, Willén C, Sunnerhagen KS. Kinematic variables quantifying upper-extremity performance after stroke during reaching and drinking from a glass. Neurorehabil Neural Repair 25: 71-80, 2011.

Murphy TH, Corbett D. Plasticity during stroke recovery: from synapse to behaviour. Nat Rev Neurosci 10: 861-872, 2009.

Nardone A, Schieppati M. Reflex contribution of spindle group Ia and II afferent input to leg muscle spasticity as revealed by tendon vibration in hemiparesis. Clin Neurophysiol 116: 13701381, 2005.

Nathan RH. Functional electrical stimulation of the upper limb: charting the forearm surface. Med Biol Eng Comput 17: 729-736, 1979.

Niu CM, Bao Y, Zhuang C, Li S, Wang T, Cui L, Xie Q, Lan N. Synergy-Based FES for Post-Stroke Rehabilitation of Upper-Limb Motor Functions. IEEE Trans Neural Syst Rehabil Eng 27: 256-264, 2019.

Olesh E V, Pollard BS, Gritsenko V. Gravitational and Dynamic Components of Muscle Torque Underlie Tonic and Phasic Muscle Activity during Goal-Directed Reaching. Frontiers in human neuroscience 11: 1-12, 2017. 
Olesh EV, Yakovenko S, Gritsenko V. Automated Assessment of Upper Extremity Movement Impairment due to Stroke. PLoS ONE 9: e104487, 2014.

Owen M, Ingo C, Dewald JPA. Upper Extremity Motor Impairments and Microstructural Changes in Bulbospinal Pathways in Chronic Hemiparetic Stroke. Front Neurol 8: 257, 2017.

Paci M, Nannetti L, Rinaldi LA. Glenohumeral subluxation in hemiplegia: An overview. $J$ Rehabil Res Dev 42: 557-568, 2005.

Papaxanthis C, Pozzo T, McIntyre J. Kinematic and dynamic processes for the control of pointing movements in humans revealed by short-term exposure to microgravity. Neuroscience 135: 371-383, 2005.

Patton JL, Stoykov ME, Kovic M, Mussa-Ivaldi FA. Evaluation of robotic training forces that either enhance or reduce error in chronic hemiparetic stroke survivors. Exp Brain Res 168: 368383, 2006.

Pelton T, van Vliet P, Hollands K. Interventions for improving coordination of reach to grasp following stroke: a systematic review. Int J Evid Based Healthc 10: 89-102, 2012.

Pigeon P, Bortolami SB, DiZio P, Lackner JR. Coordinated Turn-and-Reach Movements. I. Anticipatory Compensation for Self-Generated Coriolis and Interaction Torques. Journal of Neurophysiology 89: 276-289, 2003.

Pollock A, Farmer SE, Brady MC, Langhorne P, Mead GE, Mehrholz J, van Wijck F. Interventions for improving upper limb function after stroke. Cochrane Database Syst Rev CD010820, 2014a.

Pollock A, Farmer SE, Brady MC, Langhorne P, Mead GE, Mehrholz J, van Wijck F. Interventions for improving upper limb function after stroke. Cochrane Database Syst Rev CD010820, 2014b.

Popović Maneski L, Topalović I, Jovičić N, Dedijer S, Konstantinović L, Popović DB. Stimulation map for control of functional grasp based on multi-channel EMG recordings. Med Eng Phys 38: 1251-1259, 2016.

Porter R, Whelan J. Human Muscle Fatigue: Physiological Mechanisms. John Wiley \& Sons, 2009.

Puig J, Blasco G, Daunis-I-Estadella J, Thomalla G, Castellanos M, Figueras J, Remollo S, van Eendenburg C, Sánchez-González J, Serena J, Pedraza S. Decreased corticospinal tract fractional anisotropy predicts long-term motor outcome after stroke. Stroke 44: 2016-2018, 2013.

Quandt F, Hummel FC. The influence of functional electrical stimulation on hand motor recovery in stroke patients: a review. Exp Transl Stroke Med 6: 9, 2014. 
Riley NA, Bilodeau M. Changes in upper limb joint torque patterns and EMG signals with fatigue following a stroke. Disabil Rehabil 24: 961-969, 2002.

Ring H, Rosenthal N. Controlled study of neuroprosthetic functional electrical stimulation in sub-acute post-stroke rehabilitation. J Rehabil Med 37: 32-36, 2005.

Robertson GE, Caldwell GE, Hamill J, Kamen G, Whittlesey S. Research Methods in Biomechanics. Second edition. Champaign, Illinois: Human Kinetics, Inc., 2013.

Roh J, Rymer WZ, Perreault EJ, Yoo SB, Beer RF. Alterations in upper limb muscle synergy structure in chronic stroke survivors. J Neurophysiol 109: 768-781, 2013.

Rothi LJ, Horner J. Restitution and substitution: two theories of recovery with application to neurobehavioral treatment. J Clin Neuropsychol 5: 73-81, 1983.

Russo M, D'Andola M, Portone A, Lacquaniti F, D'Avella A. Dimensionality of joint torques and muscle patterns for reaching. Frontiers in computational neuroscience 8: 1-21, 2014.

Sainburg RL. Evidence for a dynamic-dominance hypothesis of handedness. Exp Brain Res 142: 241-258, 2002.

Sainburg RL, Ghilardi MF, Poizner H, Ghez C. Control of limb dynamics in normal subjects and patients without proprioception. Journal of Neurophysiology 73: 820-835, 1995.

Sainburg RL, Kalakanis D. Differences in control of limb dynamics during dominant and nondominant arm reaching. J Neurophysiol 83: 2661-2675, 2000.

Sakai ST, Davidson AG, Buford JA. Reticulospinal neurons in the pontomedullary reticular formation of the monkey (Macaca fascicularis). Neuroscience 163: 1158-1170, 2009.

Sanchez RJ, Liu J, Rao S, Shah P, Smith R, Rahman T, Cramer SC, Bobrow JE, Reinkensmeyer DJ. Automating Arm Movement Training Following Severe Stroke: Functional Exercises With Quantitative Feedback in a Gravity-Reduced Environment. IEEE Transactions on Neural Systems and Rehabilitation Engineering 14: 378-389, 2006.

Sangani SG, Starsky AJ, McGuire JR, Schmit BD. Multijoint reflexes of the stroke arm: neural coupling of the elbow and shoulder. Muscle Nerve 36: 694-703, 2007.

Sangari S, Perez MA. Imbalanced Corticospinal and Reticulospinal Contributions to Spasticity in Humans with Spinal Cord Injury. J Neurosci 39: 7872-7881, 2019.

Santello M, Lang CE. Are movement disorders and sensorimotor injuries pathologic synergies? When normal multi-joint movement synergies become pathologic. Front Hum Neurosci 8: 1050, 2014.

Saposnik G, Cohen LG, Mamdani M, Pooyania S, Ploughman M, Cheung D, Shaw J, Hall J, Nord P, Dukelow S, Nilanont Y, De Los Rios F, Olmos L, Levin M, Teasell R, Cohen A, Thorpe K, Laupacis A, Bayley M, Stroke Outcomes Research Canada. Efficacy and safety 
of non-immersive virtual reality exercising in stroke rehabilitation (EVREST): a randomised, multicentre, single-blind, controlled trial. Lancet Neurol 15: 1019-1027, 2016a.

Saposnik G, Cohen LG, Mamdani M, Pooyania S, Ploughman M, Cheung D, Shaw J, Hall $\mathbf{J}$, Nord P, et al. Efficacy and safety of non-immersive virtual reality exercising in stroke rehabilitation (EVREST): a randomised, multicentre, single-blind, controlled trial. The Lancet Neurology 15: 1019-1027, $2016 \mathrm{~b}$.

Schaechter JD, Fricker ZP, Perdue KL, Helmer KG, Vangel MG, Greve DN, Makris N. Microstructural status of ipsilesional and contralesional corticospinal tract correlates with motor skill in chronic stroke patients. Hum Brain Mapp 30: 3461-3474, 2009.

Schulz R, Park C-H, Boudrias M-H, Gerloff C, Hummel FC, Ward NS. Assessing the integrity of corticospinal pathways from primary and secondary cortical motor areas after stroke. Stroke 43: 2248-2251, 2012.

Schwarz A, Kanzler CM, Lambercy O, Luft AR, Veerbeek JM. Systematic Review on Kinematic Assessments of Upper Limb Movements After Stroke. Stroke 50: 718-727, 2019.

Scott SH, Norman KE. Computational approaches to motor control and their potential role for interpreting motor dysfunction: Current Opinion in Neurology 16: 693-698, 2003.

Seidler RD, Bernard JA, Burutolu TB, Fling BW, Gordon MT, Gwin JT, Kwak Y, Lipps DB. Motor control and aging: Links to age-related brain structural, functional, and biochemical effects. Neuroscience \& Biobehavioral Reviews 34: 721-733, 2010.

Shabbott BA, Sainburg RL. Differentiating Between Two Models of Motor Lateralization. Journal of Neurophysiology 100: 565-575, 2008.

Shaffer SW, Harrison AL. Aging of the Somatosensory System: A Translational Perspective. Physical Therapy 87: 193-207, 2007.

Sheean G. The pathophysiology of spasticity. Eur J Neurol 9 Suppl 1: 3-9; dicussion 53-61, 2002.

Smith C, Sun M, Kenney L, Howard D, Luckie H, Waring K, Taylor P, Merson E, Finn S, Cotterill S. A Three-Site Clinical Feasibility Study of a Flexible Functional Electrical Stimulation System to Support Functional Task Practice for Upper Limb Recovery in People With Stroke. Front Neurol 10, 2019.

Sousa ASP, Mesquita IA, Costa da Silva CI, Silva A, Macedo R, Imatz-Ojanguren E, Hernandez E, Keller T, Moreira J, Pereira da Fonseca PF, Santos R. Optimal Multifield Functional Electrical Stimulation Parameters for the "Turn on the Light" Task and Related Upper Limb Kinematics Repeatability in Poststroke Subjects. Arch Phys Med Rehabil 102: 1180-1190, 2021. 
Suethanapornkul S, Kuptniratsaikul PS, Kuptniratsaikul V, Uthensut P, Dajpratha P, Wongwisethkarn J. Post stroke shoulder subluxation and shoulder pain: a cohort multicenter study. J Med Assoc Thai 91: 1885-1892, 2008.

Sukal TM, Ellis MD, Dewald JPA. Shoulder abduction-induced reductions in reaching work area following hemiparetic stroke: neuroscientific implications. Exp Brain Res 183: 215-223, 2007.

Sullivan JE, Crowner BE, Kluding PM, Nichols D, Rose DK, Yoshida R, Pinto Zipp G. Outcome Measures for Individuals With Stroke: Process and Recommendations From the American Physical Therapy Association Neurology Section Task Force. Phys Ther 93: 13831396, 2013.

Sun M, Kenney L, Smith C, Waring K, Luckie H, Liu A, Howard D. A novel method of using accelerometry for upper limb FES control. Med Eng Phys 38: 1244-1250, 2016.

Sun M, Smith C, Howard D, Kenney L, Luckie H, Waring K, Taylor P, Merson E, Finn S. FES-UPP: A Flexible Functional Electrical Stimulation System to Support Upper Limb Functional Activity Practice. Front Neurosci 12: 449, 2018.

Talkington WJ, Pollard BS, Olesh EV, Gritsenko V. Multifunctional Setup for Studying Human Motor Control Using Transcranial Magnetic Stimulation, Electromyography, Motion Capture, and Virtual Reality. JoVE 52906, 2015.

Thrasher TA, Zivanovic V, McIlroy W, Popovic MR. Rehabilitation of reaching and grasping function in severe hemiplegic patients using functional electrical stimulation therapy.

Neurorehabil Neural Repair 22: 706-714, 2008.

Twitchell TE. The restoration of motor function following hemiplegia in man. Brain 74: 443$480,1951$.

Vafadar AK, Côté JN, Archambault PS. Effectiveness of functional electrical stimulation in improving clinical outcomes in the upper arm following stroke: a systematic review and metaanalysis. Biomed Res Int 2015: 729768, 2015.

Vidaurre C, Klauer C, Schauer T, Ramos-Murguialday A, Müller K-R. EEG-based BCI for the linear control of an upper-limb neuroprosthesis. Med Eng Phys 38: 1195-1204, 2016.

Virani Salim S., Alonso Alvaro, Aparicio Hugo J., Benjamin Emelia J., Bittencourt Marcio S., Callaway Clifton W., Carson April P., Chamberlain Alanna M., Cheng Susan, Delling Francesca N., Elkind Mitchell S.V., Evenson Kelly R., Ferguson Jane F., Gupta Deepak K., Khan Sadiya S., Kissela Brett M., Knutson Kristen L., Lee Chong D., Lewis Tené T., Liu Junxiu, Loop Matthew Shane, Lutsey Pamela L., Ma Jun, Mackey Jason, Martin Seth S., Matchar David B., Mussolino Michael E., Navaneethan Sankar D., Perak Amanda Marma, Roth Gregory A., Samad Zainab, Satou Gary M., Schroeder Emily B., Shah Svati H., Shay Christina M., Stokes Andrew, VanWagner Lisa B., Wang Nae-Yuh, Tsao Connie W., null null. Heart Disease and Stroke Statistics-2021 Update. Circulation 143: e254-e743, 2021. 
Vitoonpong T, Chang K-V. Shoulder Subluxation [Online]. In: StatPearls. StatPearls Publishinghttp://www.ncbi.nlm.nih.gov/books/NBK507847/ [30 Jun. 2021].

Volpe BT, Krebs HI, Hogan N. Is robot-aided sensorimotor training in stroke rehabilitation a realistic option?: Current Opinion in Neurology 14: 745-752, 2001.

Volpe BT, Krebs HI, Hogan N, Edelstein L, Diels C, Aisen M. A novel approach to stroke rehabilitation: Robot-aided sensorimotor stimulation. Neurology 54: 1938-1944, 2000.

Volpe BT, Krebs HI, Hogan N, Edelsteinn L, Diels CM, Aisen ML. Robot training enhanced motor outcome in patients with stroke maintained over 3 years. Neurology 53: 1874-1874, 1999.

Wang RY, Chan RC, Tsai MW. Functional electrical stimulation on chronic and acute hemiplegic shoulder subluxation. Am J Phys Med Rehabil 79: 385-390; quiz 391-394, 2000.

Wang R-Y, Yang Y-R, Tsai M-W, Wang WTJ, Chan R-C. Effects of functional electric stimulation on upper limb motor function and shoulder range of motion in hemiplegic patients. Am J Phys Med Rehabil 81: 283-290, 2002.

Wang W, Dounskaia N. Neural control of arm movements reveals a tendency to use gravity to simplify joint coordination rather than to decrease muscle effort. Neuroscience 339: 418-432, 2016.

Wilkins KB, Dewald JPA, Yao J. Intervention-induced changes in neural connectivity during motor preparation may affect cortical activity at motor execution. Sci Rep 10: 7326, 2020.

Wilson VJ, Zarzecki P, Schor RH, Isu N, Rose PK, Sato H, Thomson DB, Umezaki T. Cortical influences on the vestibular nuclei of the cat. Exp Brain Res 125: 1-13, 1999.

Winter DA. Biomechanics and Motor Control of Human Movement [Online]. 4th ed. Wiley.https://www.wiley.com/enus/Biomechanics+and+Motor+Control+of+Human+Movement $\% 2 \mathrm{C}+4$ th + Edition-p9780470398180 [2 Jan. 2020].

Wolf SL, Catlin PA, Ellis M, Archer AL, Morgan B, Piacentino A. Assessing Wolf motor function test as outcome measure for research in patients after stroke. Stroke 32: 1635-1639, 2001a.

Wolf SL, Catlin PA, Ellis M, Archer AL, Morgan B, Piacentino A. Assessing Wolf Motor Function Test as Outcome Measure for Research in Patients After Stroke. Stroke 32: 1635-1639, $2001 b$.

Wolf SL, Thompson PA, Winstein CJ, Miller JP, Blanton SR, Nichols-Larsen DS, Morris DM, Uswatte G, Taub E, Light KE, Sawaki L. The EXCITE stroke trial: comparing early and delayed constraint-induced movement therapy. Stroke 41: 2309-2315, 2010a. 
Wolf SL, Thompson PA, Winstein CJ, Miller JP, Blanton SR, Nichols-Larsen DS, Morris DM, Uswatte G, Taub E, Light KE, Sawaki L. The EXCITE stroke trial: comparing early and delayed constraint-induced movement therapy. Stroke 41: 2309-2315, 2010b.

Yang K, Meadmore K, Freeman C, Grabham N, Hughes A-M, Wei Y, Torah R, GlancGostkiewicz M, Beeby S, Tudor J. Development of User-Friendly Wearable Electronic Textiles for Healthcare Applications. Sensors (Basel) 18, 2018.

Yoo JS, Choi BY, Chang CH, Jung YJ, Kim SH, Jang SH. Characteristics of injury of the corticospinal tract and corticoreticular pathway in hemiparetic patients with putaminal hemorrhage. BMC Neurology 14: undefined-undefined, 2014.

You G, Liang H, Yan T. Functional electrical stimulation early after stroke improves lower limb motor function and ability in activities of daily living. NeuroRehabilitation 35: 381-389, 2014.

Young SJ, Pratt J, Chau T. Target-Directed Movements at a Comfortable Pace: Movement Duration and Fitts's Law. Journal of Motor Behavior 41: 339-346, 2009.

Yozbatiran N, Der-Yeghiaian L, Cramer SC. A Standardized Approach to Performing the Action Research Arm Test. Neurorehabil Neural Repair 22: 78-90, 2008.

Zaaimi B, Edgley SA, Soteropoulos DS, Baker SN. Changes in descending motor pathway connectivity after corticospinal tract lesion in macaque monkey. Brain 135: 2277-2289, 2012. 